Florida International University FIU Digital Commons

FIU Electronic Theses and Dissertations

University Graduate School

$11-17-2017$

\title{
Associations of HIV Risk Behaviors among Older Latinas and their Adult Daughters
}

Gira J. Ravelo

Florida International University, grave001@fiu.edu

DOI: $10.25148 /$ etd.FIDC004066

Follow this and additional works at: https:// digitalcommons.fiu.edu/etd

Part of the Community Health Commons, Community Health and Preventive Medicine Commons, Epidemiology Commons, Immune System Diseases Commons, Other Social and

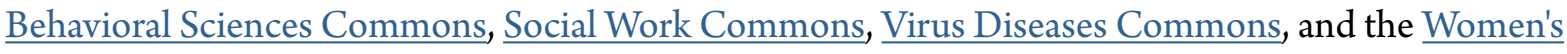
Health Commons

\section{Recommended Citation}

Ravelo, Gira J., "Associations of HIV Risk Behaviors among Older Latinas and their Adult Daughters" (2017). FIU Electronic Theses and Dissertations. 3494.

https://digitalcommons.fiu.edu/etd/3494

This work is brought to you for free and open access by the University Graduate School at FIU Digital Commons. It has been accepted for inclusion in FIU Electronic Theses and Dissertations by an authorized administrator of FIU Digital Commons. For more information, please contact dcc@fiu.edu. 


\title{
FLORIDA INTERNATIONAL UNIVERSITY
}

Miami, Florida

\section{ASSOCIATIONS OF HIV RISK BEHAVIORS AMONG OLDER LATINAS AND THEIR ADULT DAUGHTERS}

\author{
A dissertation submitted in partial fulfillment of \\ the requirements for the degree of \\ DOCTOR OF PHILOSOPHY \\ in \\ SOCIAL WELFARE \\ by
}

Gira J. Ravelo 
To: Dean Thomás R. Guilarte

Robert Stempel College of Public Health and Social Work

This dissertation, written by Gira J. Ravelo, and entitled Associations of HIV Risk Behaviors among Older Latinas and Their Adult Daughters, having been approved in respect to style and intellectual content, is referred to you for judgement.

We have read this dissertation and recommend that it be approved.

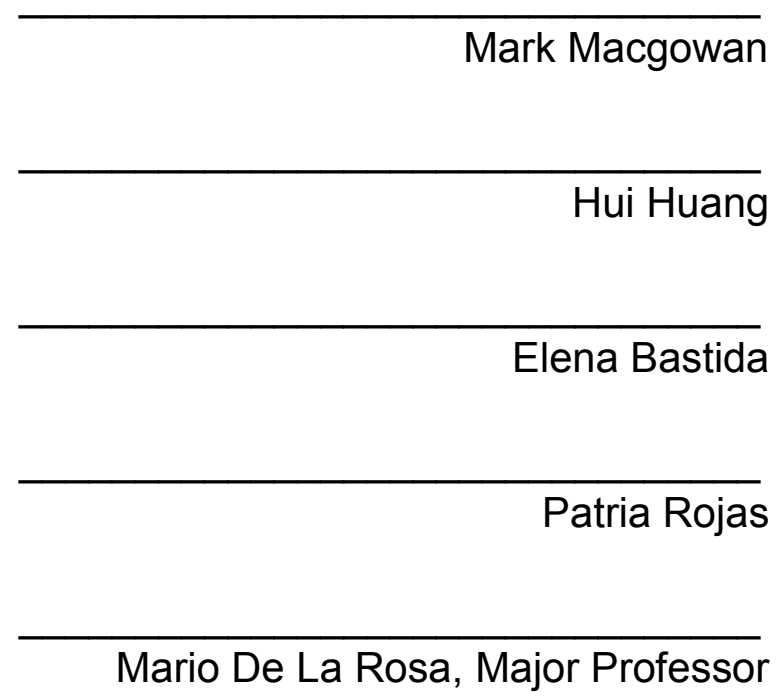

Date of Defense: November 17, 2017

The dissertation of Gira J. Ravelo is approved.

Dean Thomás R. Guilarte Robert Stempel College of Public Health and Social Work

Andrés G. Gil Vice President for Research and Economic Development and Dean of the University Graduate School

Florida International University, 2017 


\section{DEDICATION}

I dedicate this dissertation to my family. My sisters Melissa, and Mercedes (Mechy), I love you both always whether near or far, let's keep the torch burning sisters! To my stepdad Luis, for being a pillar of strength whenever called upon; and a special dedication to Ana Maria, for hanging on through the good and bad, especially during this very challenging time.

Finally, to the one who has sacrificed the most to give me love, support, strength, and life; for all you gave and gave-up to see me succeed, I dedicate this mother-daughter dissertation to my very own loving mother, Angela C. TaverasRavelo. Thank you mommy, for everything you have done to always keep us safe and make us strong.

Your loving daughter.

Gira. 


\section{ACKNOWLEDGMENTS}

I'd like to acknowledge my chair, director, and major professor, Dr. Mario De La Rosa for always providing me with resources and mentorship and being the best Chair anyone could ask for. Thank you to my committee members $\mathrm{Dr}$. Mark Macgowan, Dr. Hui Huang, and a very supportive Dr. Elena Bastida. Thank you Dr. Richard Beaulaurier for planting the seed and to Dr. Barbara Thomlison for keeping me focused. Finally, a very special acknowledgement to my fifth committee member, Dr. Patria Rojas, for being the first to inspire me and believe so deeply in me, without you and your steady persistence and faith, this would never have come to pass; you have been a boss, a teacher, a mentor, an ally, a committee member, and most of all, a friend.

I would like to acknowledge the entire CRUSADA staff for all their work and countless hours of support. Also, a big thanks to all my friends for their endless encouragement, Elizabeth, Antonina, Maricarmen, Barry, Elena, Adi, and especially Mariana, without all your timeless efforts, sacrifices, and enduring mentorship and friendship, I may have never crossed over the finish line. A very special thanks to my long-time best-friend Isela Imai, though far away, your warm support always felt near and was always there when I need it most.

To conclude, l'd like to acknowledge the National Institute of Minority Health and Health Disparities for their financial contributions to my academic and professional growth. 


\section{ABSTRACT OF THE DISSERTATION \\ ASSOCIATIONS OF HIV RISK BEHAVIORS AMONG \\ OLDER LATINAS AND THEIR ADULT DAUGHTERS}

by

Gira J. Ravelo

Florida International University, 2017

Miami, Florida

Professor Mario De La Rosa, Major Professor

This dissertation examines the associations between adult Latina

daughters' HIV risk behaviors and that of their older Latina mothers' HIV risk behaviors. The central hypothesis of the study is that adult Latina daughters' influence their older mothers' behaviors and that such behaviors interact with the level of their attachment.

Older Latinas are a vulnerable population that face cultural risk factors, health care provider bias, and engage in low rates of HIV testing. The primary mode of HIV infection for Latinas is through heterosexual contact. Yet, traditional gender norms such as marianismo belief place pressure on Latina women to be submissive to their partners, disregard their infidelities, and not attempt to negotiate condom use, increasing the risk of HIV infection among Latinas via heterosexual sex with their primary partners.

Efforts by the CDC to increase HIV testing have emerged as a critical part of HIV prevention. However, research has indicated that older Latinas are less likely to speak to a health care provider about safe sex practices or HIV testing 
than their younger counterparts. Despite health care providers having strong influences over Latinas' decisions to perform health related tests, physicians are not endorsing HIV testing for their older patients. This in part, leads to a disproportionate number of late stage diagnoses among older adults. Evidence suggest that adult daughters are a source of support and assistance in healthrelated issues for their aging mothers and are uniquely positioned to influence their mothers' health-related behaviors and decisions. Additionally, motherdaughter attachment has been found to be a protective factor to other risky health behaviors.

The present study (1) Examined the association between recurrent HIV risk behaviors of adult Latina daughters and those of their older Latina mothers and (2) examined the moderating effect mother-daughter attachment have on those associations. No statistical significance was found in examining the association between adult Latina daughters and their older mothers, nor was attachment found to be a moderator on those associations. Findings show culture may be the significant factor influencing the lack of association between adult Latina daughters and their older mothers. Results add to the literature and scientific field of social work and public health. Additionally promoting studies on cultural barriers that may exist in adult Latina daughter and older mother upward communication as techniques to promote healthy behaviors among older Latinas. 


\section{TABLE OF CONTENTS}

CHAPTER

PAGE

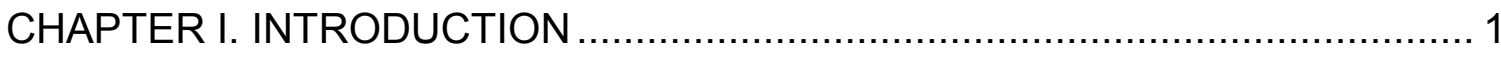

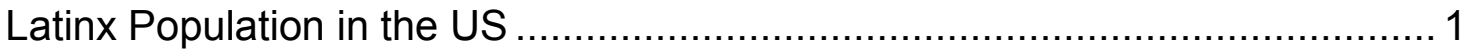

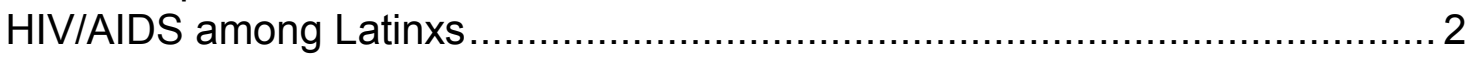

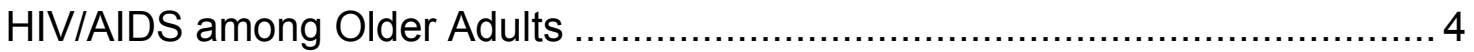

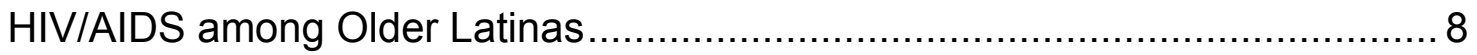

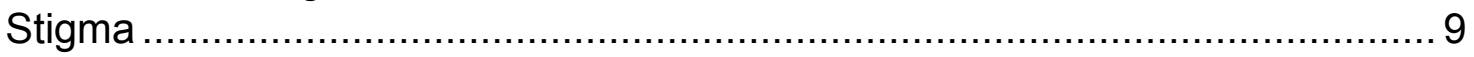

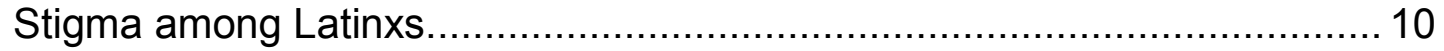

Present Study Research Question ....................................................... 11

CHAPTER II. REVIEW OF RELEVANT CONTRUCTS …............................. 13

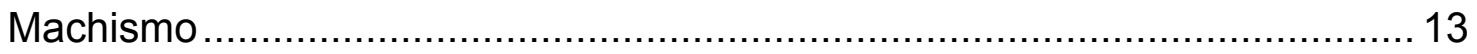

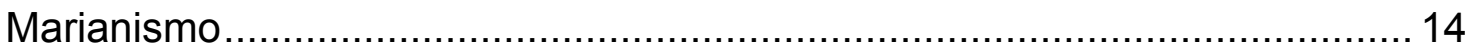

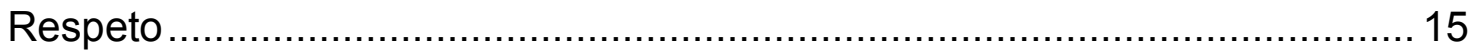

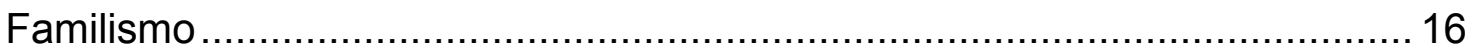

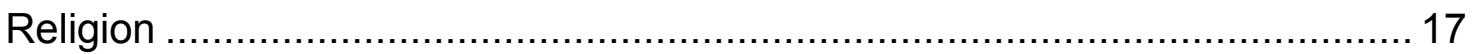

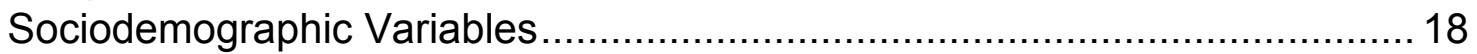

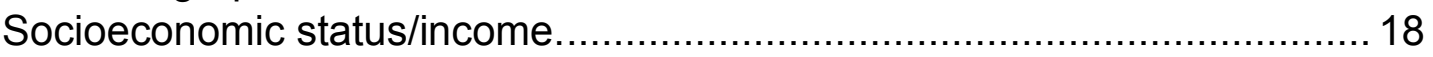

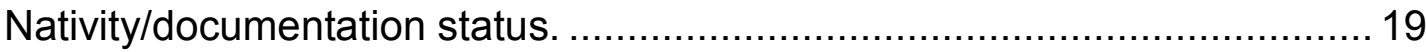

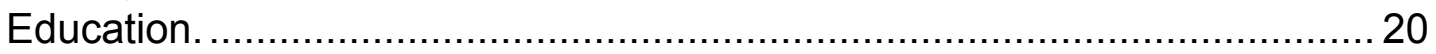

Sex under the Influence of Alcohol and other Drugs ................................. 21

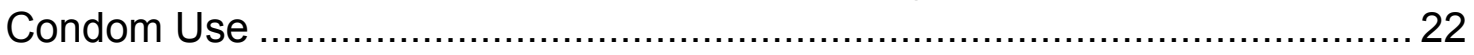

Communicating with Older Patient / HIV Counseling ................................... 23

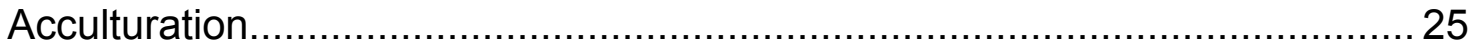

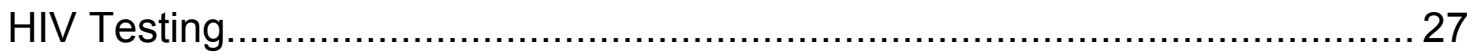

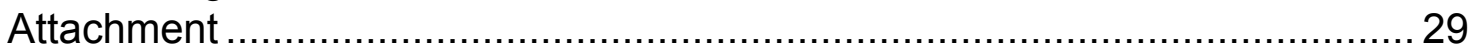

Daughter-Initiated Communication on Health-Related Behaviors .................. 31

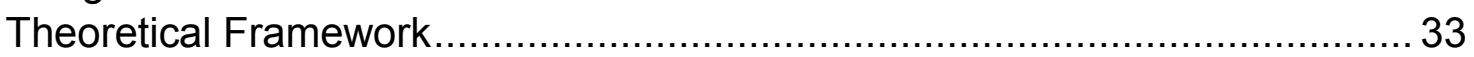

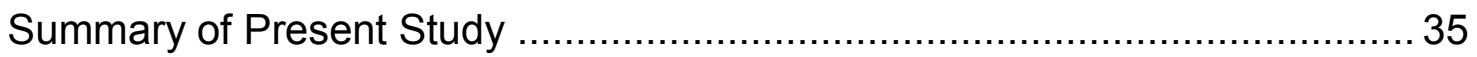

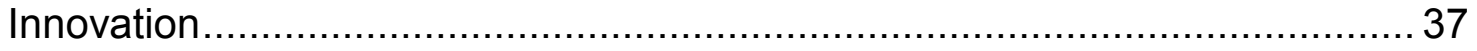

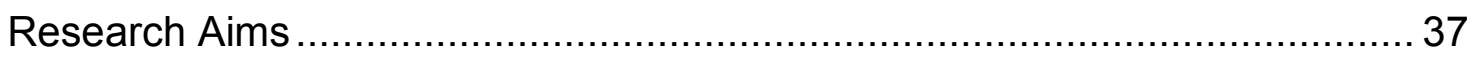

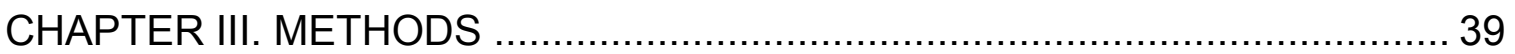

Summary of Parent Study and Data Source ........................................... 39

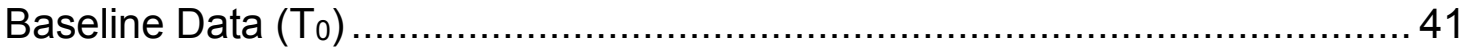

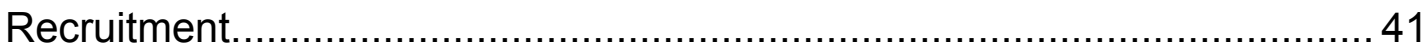

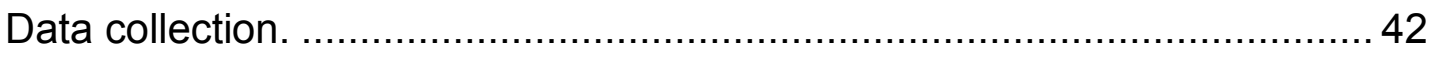

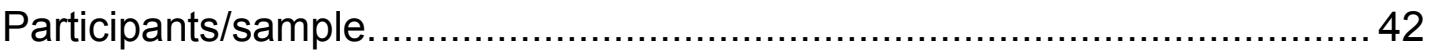

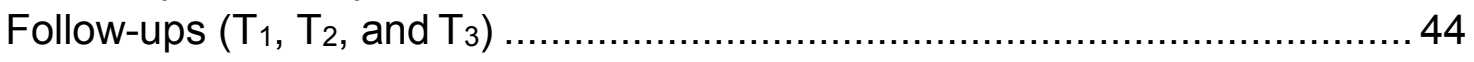




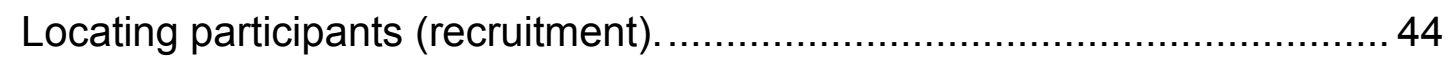

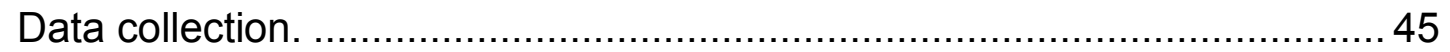

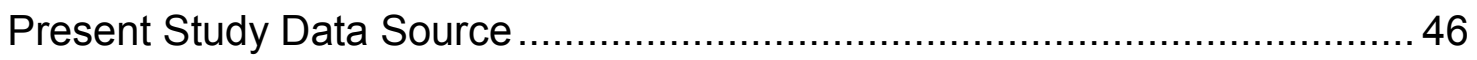

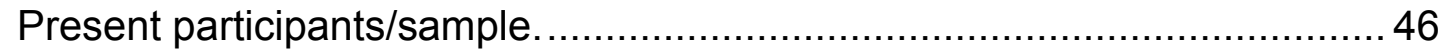

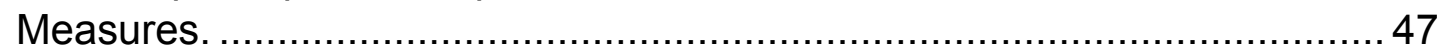

Inventory of parent and peer attachment (ippa) (attachment)................. 47

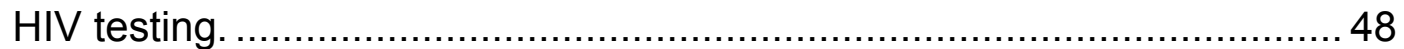

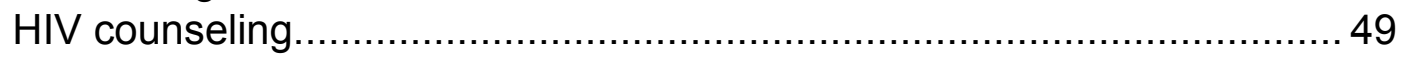

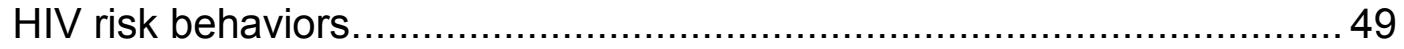

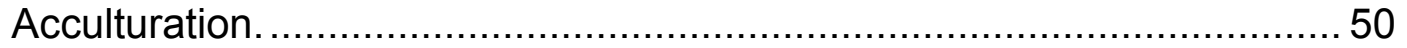

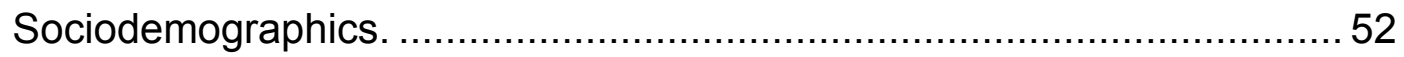

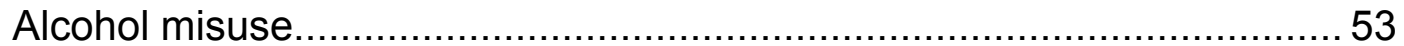

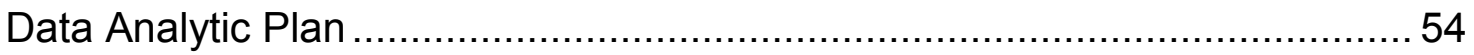

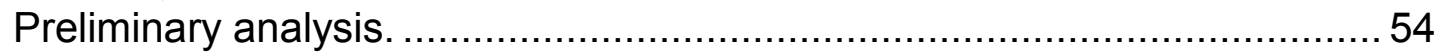

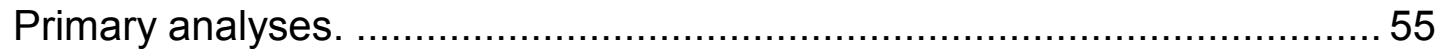

Human Participants Consideration ...................................................... 58

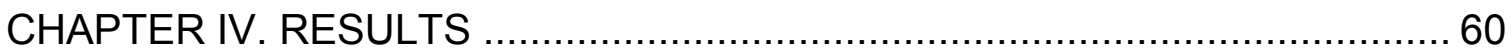

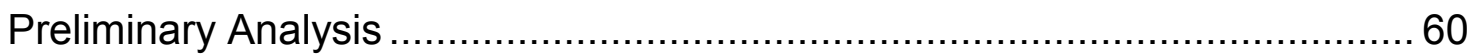

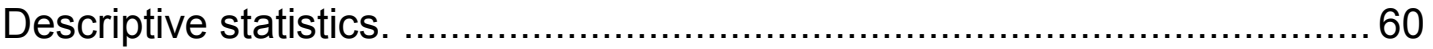

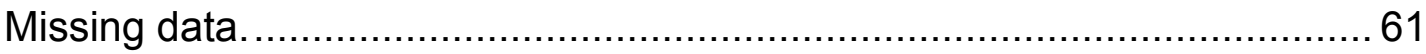

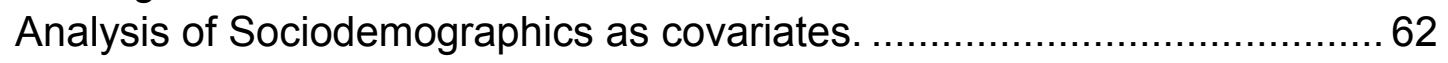

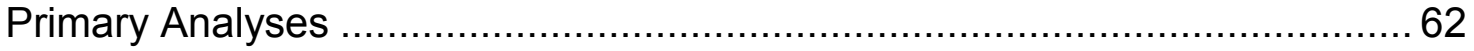

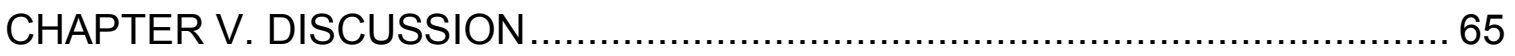

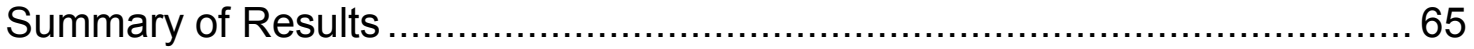

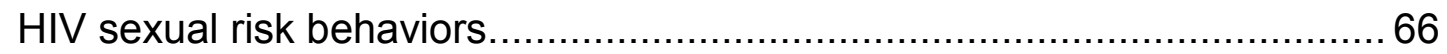

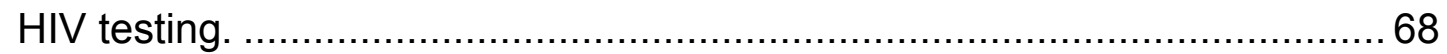

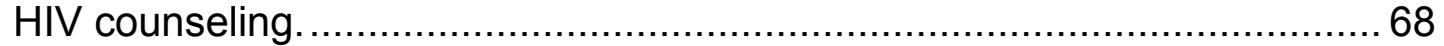

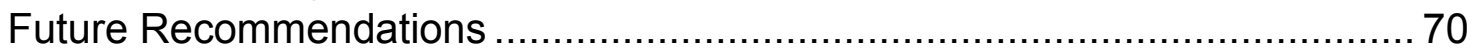

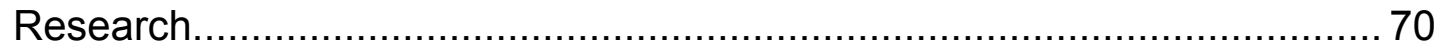

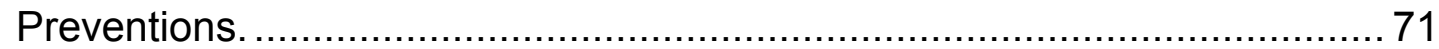

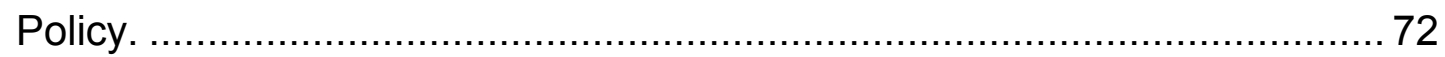

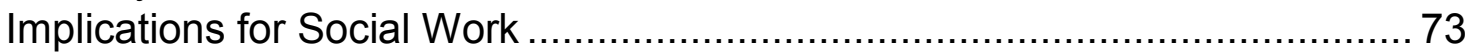

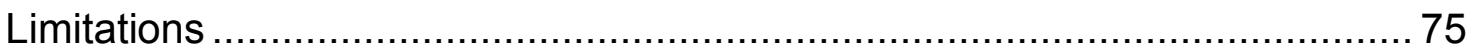

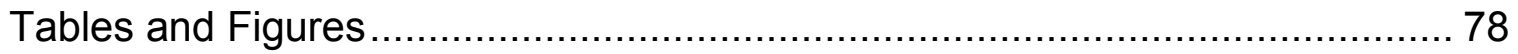

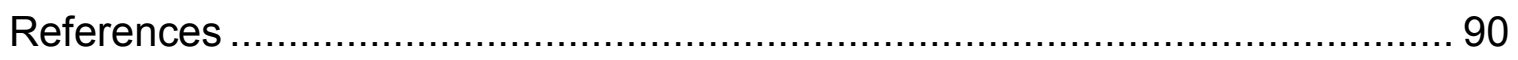

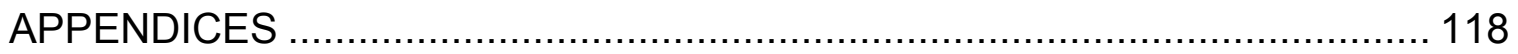

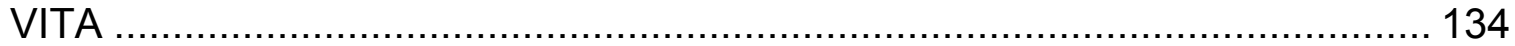




\section{LIST OF TABLES}

TABLE PAGE

1. Inclusion Criteria for Current Study ............................................... 79

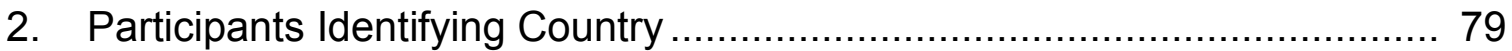

3. Descriptive Statistic for Mothers and Daughters ................................... 80

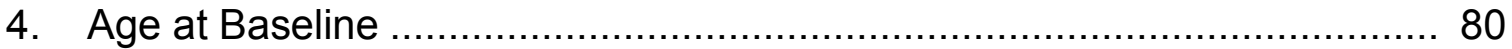

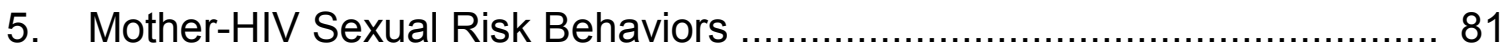

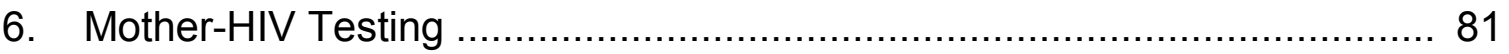

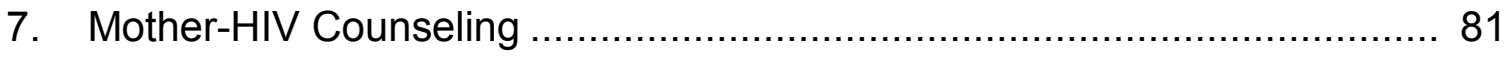

8. Daughter-HIV Sexual Risk Behaviors ….................................................. 82

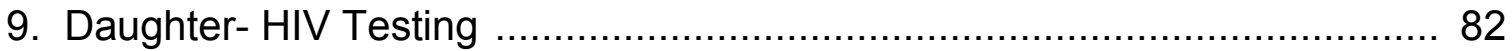

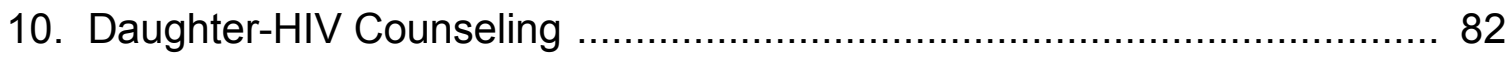




\section{LIST OF FIGURES}

FIGURE

PAGE

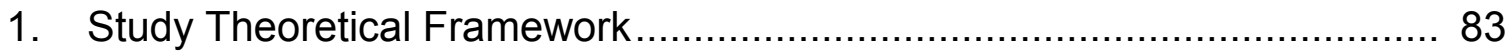

2. Study Research Model ................................................................ 84

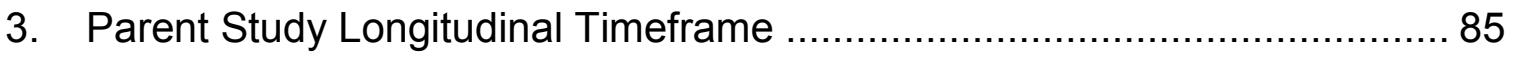

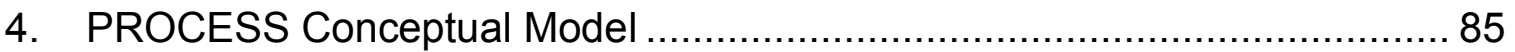

5. To: Recurrent Cross-sectional Moderator Model...................................... 86

6. T1: Recurrent Cross-sectional Moderator Model...................................... 87

7. $\mathrm{T}_{2}$ : Recurrent Cross-sectional Moderator Model....................................... 88

8. $T_{3}:$ Recurrent Cross-sectional Moderator Model...................................... 89 


\section{CHAPTER I. INTRODUCTION}

\section{Latinx Population in the US}

The 58 million Latinxs in the United States (US) represent $18 \%$ of the nation's population; they are the largest ethnic minority group in the US and second largest racial or ethnic group behind to non-Latinx Whites (Flores, 2017). Since 1970, the Latinx population in the US has increased 592\%; between 2000 and 2010 , the US population grew by 27.3 million, Latinxs accounted for more than half of that growth (15.2 million) (Pew Research Center, 2014). In 2015, the US Census Bureau reports that this population is projected to increase to 119 million by 2060 (US Census Bureau, 2015). Based on this projected increase, the US Latinx population will comprise $29 \%$ of the total US population by 2060 (US Census Bureau, 2015). Similarly, the older Latino population is also growing. In 2015, the Hispanic median age was up to age 28 compared to 25 in the year 2000. Although the median age for non-Latinx White was 43 , foreign-born Latinxs had a median age of 42, which was up from 33 in 2000 (Pew, 2017).

In this study, the term Latinxs refers to both Latino men and Latina women, traditionally used as Latinos and is also being used interchangeably with the term Hispanics. The term refers to individuals, and their descendants, who were (a) born in the Spanish speaking countries of the Americas, including North (Mexico), Central, and South America—and the Caribbean—or (b) self-identify as Latinxs, regardless of race.

In 2014, the American Association of Retired Persons (AARP) reported

that Latinxs age 50 and over represent $9 \%$ of the US population (2014). Yet, the 
percentage of older Latinxs is projected to rise every year; reaching $9 \%$ by 2017 (American Association of Retired Persons [AARP], 2014). The growth in ethnic/racial minority populations is changing the country's composition; the US Census reported that by 2060 the 50 plus Latinx population will reach over 42 million, making up $24 \%$ of the US population (The Nielsen Company, 2015). The US Census Bureau reports that by 2020 , “more than half of the nation's children are expected to be part of a minority race or ethnic group" (para. 3); by 2044, the country is expected to become a "majority-minority" (para. 4), where no single group will hold majority share of the total. Additionally, by 2060 the ethnic/racial minority population is expected to rise to $56 \%$ of the total population (US Census Bureau, 2015)

\section{HIV/AIDS among Latinxs}

In 2011 Latinxs represented $16 \%$ of the US population and accounted for $21 \%$ of all new AIDS diagnoses (Keiser Family Foundation [KFF], 2014); in 2013, Latinxs comprised 21\% of all people living with HIV (CDC, 2017a). By 2015, Latinxs accounted for $18 \%$ of the total population and for $24 \%$ of all newly diagnosed HIV infections (CDC, 2017b). About $70 \%$ of all diagnosed HIV infection among Latinxs occur in gay and bisexual men; these men are account for $85 \%$ of infections among Latinos. Among Latinas, $90 \%$ of HIV infection occur from heterosexual contact (CDC, 2017b). Generally, the growing Latinx population is being infected at nearly three times the rate of non-Latinx Whites (CDC, 2017a). Specifically, in 2010, rates of new HIV infection among Latinos and Latinas were 2.9 and 4.2 times, respectively, then that of their non-Latinx 
White counterparts (CDC, 2015a). Among older Latinxs, the rate is 5 times higher (Linley, Prejean, An, Chen, \& Hall, 2012). Additionally, Latinxs and older adults are both more likely to receive a late HIV diagnosis, compared to nonLatinx White (Ellman, Sexton, Warshafsky, Sobieszczyk, \& Morrison, 2014; Sheehan, Trepka, Fennie, \& Maddox, 2015; Sheehan et al., 2017), presenting a critical health disparity for both older adults and Latinxs, particularly Latinas.

Much of what makes HIVIAIDS more prevalent among Latinxs is related to Latinx culture. Evidence has shown that several cultural factors contribute to the spread of HIV among Latinxs and create barriers for prevention (Beaulaurier, Craig, De La Rosa, 2009; Rojas et al., 2016). These factors include machismo (Pinel, 1994), marianismo (Marin, Tschnn, Gomez, \& Kegeles, 1993; Moreno, 2007). Additionally, social economic status, nativity, acculturation, and poor communication with health care providers (Cianelli, Ferrer, McElmurry, 2008; Dang, Giordano, \& Kim, 2012; Gonzalez-Guarda, Peragallo, Urrutia, \& Vasquez, 2008; Peragallo, 1996; Shedlin, Decena, \& Oliver-Velez, 2005). Additionally, among undocumented Latinx immigrants, fear of deportation deters them from accessing health care and preventative services, which are already scarce in many Latinx communities. Even in cases when proof of legal status is not a requirement for service delivery, misinformation about rights to health care and lack of trust in the system prevent this population from accessing much needed health care services (Derose, Escarce, \& Lurie, 2007). 


\section{HIV/AIDS among Older Adults}

The US population is getting older. In fact, the US Centers for Disease Control and Prevention (CDC) reported that the number of Americans aged 100 and over rose from 50,281 in 2000 to 72,197 in 2014 , an increase of $43.6 \%$ (CDC, 2016). In addition to the growing numbers of older adults, they are also very sexually active (Lindau, Schumm, Laumann, Levinson, O'Muircheartaigh, \& Waite, 2007). According to the CDC, people ages 50 years account for approximately $45 \%$ of people living with a diagnosed HIV in the United States (CDC, 2017c). Additionally, in 2015, this same group accounted for $17 \%$ of all HIV diagnosis in the US (CDC, 2017c). The largest numbers of diagnosis (45\%) were among those 50-54 years of age (CDC, 2017c). Moreover, individuals age 50 and over accounted for $27 \%$ of the estimated 26,688 AIDS diagnoses in the US (CDC, 2015b; Effros et al., 2008). Increase in risky behaviors among older people, lack of knowledge, and lack of social media campaigns directed at this population have contributed to the rise of new HIV infections among older people (McCaig, Hardy, \& Winn, 1991; Effros et al., 2008; Jacobs \& Thomlison, 2009).

When HIV first emerged, the issue of HIV among older adults was not prioritized because older adults were not considered to be at high risk for sexually transmitted infections (STIs); however, more recent studies have proven otherwise (Cahill, \& Valadéz, 2013; Linsk, 2000; Negin et al., 2012; Poynten, Grulich, \& Templeton, 2013; Pratt, Gascoyne, Cunningham, \& Tunbridge, 2010; Simone, \& Appelbaum, 2008; Smith, Delpech, Brown, \& Rice, 2010). The literature has placed more focus on HIV and other sexually transmitted diseases 
among older adults. Such studies underscore that older adults are indeed sexually active. Yet studies have shown that older adults remain sexually active well into their 80s; they maintain sexual desires and engage in risky sexual behaviors, such as having multiple sex partners and engaging in sexual intercourse without condoms (Johnson, 2013; Falvo \& Norman, 2004; Jena, Goldman, Kamdar, Lakdawalla, \& Lu, 2010; Schick et al., 2010). In a New York study of unprotected sex among older adults age 50 to 78 ( $70 \%$ male; $29 \%$ female) with HIV, $50 \%$ reported sexual activity in the past 90 days. Almost half (49\%) of them reported having sex at least 2-3 times a week, and over 33\% admitted to engaging in unprotected anal or vaginal sex in the past 90 days ( $18 \%$ with serodiscordant partners) (Golub et al., 2010).

New medical developments have also contributed to the increasing numbers of older adults with HIVIAIDS. The current generation of older adults, has come into their older years with erectile dysfunction medications (ED meds) on the market. Use of ED meds have shown to be correlated to increases in sexually transmitted disease among users (Jena et al., 2010) including HIV. In an Ohio University survey, one-third of sexually active older adults infected with HIV reported having unprotected sex, the majority were men, reported being in an ongoing relationship, and taking ED meds (Ohio University, 2007). Additionally, the number of people aging with HIVIAIDS has increased significantly, partially due to advances in medical research and new medical developments such as highly active antiretroviral therapy (HAART) (Effros et al., 2008). Consequently, 
medical breakthroughs have creating a new public health issue of older adults with STDs/HIV who may also be experiencing other morbidities.

Adults over age 50 report having less knowledge of HIVIAIDS than that of their younger counterparts; most believe they are at less risk, and report less concern about unintentional pregnancy. Because the baby boomer generation first became sexually active during an era when condoms were mostly used as a contraceptive, they are less likely to use condoms to avoid sexually transmitted diseases and, thus, are less likely to be tested for HIV (Emlet \& Farkas, 2001; Lindau et al., 2006).

However, lack of knowledge on HIVIAIDS among older people is not exclusive to older adults themselves, it is also true of a society and health care system that holds misconception that older people are not sexually active and are therefore at low or no risk for HIV infection (Linsk, 2000; Adekeye, Heiman, Onyeabor, \& Hyacinth, 2012). Not only do physicians and health care providers have little training about HIVIAIDS among older patients, but they also experience discomfort in initiating discussions with their patients about HIVIAIDS (Harding \& Manry, 2017; Hughes, 2011; Skiest \& Keiser, 1997). In fact, older adults often experience sexual problems, which they find difficult to share with their physicians (Lindau, Leitsch, Lundberg, \& Jerome, 2006) and physician are rarely engaging older adults about sexual behaviors (Harding \& Manry, 2017; Skiest \& Keiser, 1997). Lack of communication, knowledge, and ease among these health care providers, and their older patients, create barriers for early 
detection and education. These are missed opportunities for prevention, early diagnosis, early treatment, and to reduce stigmas and taboos of testing.

Symptoms of HIV can resemble those of aging to include fatigue, weight loss, mental confusion, and dementia (Harris, 2015). Often this overlap in symptoms results in older patients receiving a late HIV diagnoses. Late detection of HIVIAIDS results in increased mortality, increase spread of the virus, weaker response to HAART medication, and higher health care costs (Mugavero, Castellano, Edelman, \& Hicks, 2007) and increased co-morbidities such as hepatitis, mental health disorders and cardiovascular disease (Lorenc, Ananthavarathan, Lorigan, Jowata, Brook, \& Banarsee, 2014). Early testing and detection is particularly important among older people, because age influences the progression of HIVIAIDS. Once diagnosed with AIDS, life expectancy of people over age 50 is an average of 7 years, compared to 12 years among those age 13-49, with older women being more likely to die within 30 days of an AIDS diagnosis (Inungu, Mokotoff, \& Kent, 2001; Lekas, Schrimshaw, \& Siegel, 2005; Nguyen \& Holodniy, 2008). Moreover, biological factors among older women increase their vulnerability to HIV infection-in comparison to their younger counterparts. Decrease in vaginal lubrication and thinning of the vaginal wall, both seen in older women, increases the likelihood of tearing along the vaginal wall, which could serve as an entry portal for HIV and STIs (Savasta, 2004; Gaeta, LaPolla, \& Melendez, 1996). A weakening of an aging immune system increases the prevalence of infection as well as a more rapid progression of HIV than in younger individuals (Coleman, 2003; Gaeta, LaPolla, \& Melendez, 1996). 
Yet, in 2017, Guo and Sims reported that older women are significantly less likely to receive HIV testing compared to men and younger counterparts.

\section{HIV/AIDS among Older Latinas}

Most older and middle age Latinas are not intravenous drug users, do not have multiple sex partners, and do not have regular blood transfusions;

therefore, they do not perceive themselves as being at high risk for HIV infection (Lopez-Quintero, Rojas, Dillon, Varga, \& De La Rosa, 2016; Ochoa \& Sampalis, 2014). Unfortunately, health providers also perceive their older patients to be at low risk for HIV as they are less likely to discuss sexual risk behaviors with them, than with their younger counterparts (Pilowsky \& Wu, 2015). Patients such as older Latinas are consequently being misdiagnosed or receiving a late HIV diagnosis and consequently not able to benefit fully from antiretroviral therapies (Cohn \& Clark, 2003).

Age, cultural, and gender sensitive HIV testing strategies are critical to preventing the spread of HIV among older Latinas. This population, that maintains cultural traditions and barriers that prevent HIV testing, is at high risk for HIV infection (Cianelli et al., 2013). Older Latinas have been described as a hidden population that faces health care provider bias, cultural risk factors, and increasing HIV infection rates (Craig, Beaulaurier, Newman, De La Rosa, \& Brennan, 2012). Linley et al. (2012) found that older people were twice as likely—and Latinxs were five times as likely—to receive a late HIV diagnosis, compared to non-Latinx Whites; thus, placing older Latinas at a great disadvantage. The CDC has recommended routine, voluntary testing for all 
adolescents and adults ages 13 to 64 (CDC, 2006). Because sexually active adults over age 64 are not included in the CDC recommendation, this population may not perceive themselves to be at risk for HIV infection. However, the Infectious Diseases Society of America has recommended routine HIV testing for all sexually active adults (Lubinski et al., 2009), targeting a larger at-risk population and encouraging testing for all adults.

\section{Stigma}

Studies have shown that stigmatizing attitudes toward people living with HIVIAIDS are linked to lower HIV testing (Brooks, Etzel, Hinojos, Henry, \& Perez, 2005; Brown, Macintyre, \& Trujillo, 2003; Mahajan et al., 2008). The Joint United Nations Programme on AIDS (UNAIDS) defines HIV stigma as a "process of devaluation of people either living with or associated with HIVIAIDS. This stigma often stems from the underlying stigmatization of sex and intravenous drug use two of the primary routes of HIV infection" (UNAIDS, 2003, p. 1). HIV stigmatization first emerged in the United States as early as the virus itself (KFF, 2012)—stigmatizing homosexual men, hemophiliacs, Haitians, and heroin drug users, later known as the 4-H Club (Bazell, 2007). Additionally, then President Ronald Regan, who for years avoided mentioning the term AIDS in public (Boffey, 1985), banned HIV positive immigrants from entering the country (US Department of Health \& Human Services [HHS], 2011), adding immigrants to the stigmatized group. In 2010, 30 years after the first cases of HIV surfaced, President Barrack Obama released the National HIVIAIDS Strategy; its vision stated that: 
The United States will become a place where new HIV infections are rare and when they do occur, every person regardless of age, gender, race/ethnicity, sexual orientation, gender identity, or socio-economic circumstance, will have unfettered access to high quality, life-extending care, free from stigma and discrimination. (National HIVIAIDS Strategy for the United States, 2010, p. 1)

Goals for the new 2020 National HIVIAIDS Strategy include tackling misconceptions, discrimination and stigma in order to break down the barriers to HIV testing, prevention, and care. Thus, a nation where HIV infected people are "free from stigma" continues to remain the vision (National HIVIAIDS Strategy for the United States, 2015, p. 1). Researchers have previously determined that HIV testing is a critical part of HIV prevention (Castilla et al., 2002; De Cock, \& Johnson, 1998; HHS, 2011; Weinhardt, Carey, Johnson, \& Bickham, 1999); however, high risk populations, such as Latinxs, are less likely to be tested due to stigma (Gilbert \& Rhodes, 2013; Mugavero et al., 2007).

\section{Stigma among Latinxs.}

Among traditional Latinx families, parents are not comfortable discussing sex or sex related issues with their children (Gil \& Vazquez, 1996). The topic of sex is considered taboo for them, however when discussion about sex do take place, often Latinx parents inadvertently give mixed and confusing messages to their children (Gil \& Vazquez, 1996). As for Latina daughters, several studies have found that they do report higher levels of communication with their mothers, compared to Latino sons, however, communication is less about risky sexual 
behaviors and more about relationships, beliefs, and values (Raffaelli \& Green, 2003; Romo, Lefkowitz, Sigman, \& Au, 2002; Villarruel, 1998). Such studies underscore the notion that Latina mothers and daughters are unlikely to have discussions about risky and protective sexual behaviors (Mena, Dillon, Mason, \& Santisteban, 2008). The present study attempts to examine whether the risky behaviors that more acculturated adult Latinas display are associated with the risky behaviors displayed by their mothers, as moderated by their relationship. Studies have shown that adult daughters may be influential in their mothers' health related behaviors (Browne \& Chan, 2012; Kratzke, Amatya, \& Vilchis, 2014; Mosavel, 2009; Mosavel, \& Genderson, 2016); thus, they may also play a role in influencing their mothers' risk and protective sexual behaviors.

\section{Present Study Research Question}

The present research study takes a step toward addressing a gap in the research literature regarding the influence that adult Latina daughters can have on their older mothers' sexual health behaviors. The study's key aims were to (a) examine the association the HIV risk behaviors of adult Latina daughters and those of their older Latina mothers and (b) examine the interaction effect of mother-daughter attachment on any associations between the HIV risk behaviors of adult Latina daughters and those of their older Latina mothers. The present study also focused on additional factors that increase HIV risk, such as substance use, multiple sex partners, and HIV testing and counseling.

The present study used a recurrent cross-sectional path analysis method of secondary data. The data originated from a longitudinal study with dyadic 
Latina mothers and daughters. The parent study collected data from four different time points, all of which were used for the present study using a quantitative approach. The longitudinal data from this parent study — which was conducted on dyads of substance using and non-using Latina mothers and daughtersprovides a unique opportunity to further examine this hard-to-reach population. Therefore, the present investigation set out to examine if associations exist between the HIV risk behaviors of adult Latina daughters and their mothers and whether these associations are moderated by attachment. 


\section{CHAPTER II. REVIEW OF RELEVANT CONTRUCTS}

\section{Machismo}

The concept of machismo is a central part of Latinx culture. It relates to the social domination and control that men have over women. Machismo contributes to gender discrimination, sexual harassment, and men's financial, legal, and psychological control of women (Pinel, 1994). The literature portrays the machismo as men with autonomy while Latinas are depicted as being dutiful to families and partners. This, is a historically traditional belief of the stereotypical Latino. Although traditional Latino men seem to have more autonomy and hold most of the power, they are also responsible for the safety of their family. The traditional Latino man "protects his wife and family from all dangers" (Gil, \& Vasquez, 1996, p. 9), making machismo a risk as well as protective cultural belief. Yet, regardless of marital status, this belief accepts, and at times, encourages men to have multiple sex partners while continuing sexual intimacy with their current partner or wife-a cultural belief that is associated with low condom use and high risk for HIV infection for both the men and their partners (Beaulaurier, Craig, \& De La Rosa, 2009; Cianelli, Ferrer, \& McElmurry, 2008; Pleck, Sonenstein, \& Ku, 1993). Because most Latinas in the US are infected via heterosexual sex with their primary partners, machismo becomes an important cultural factor to consider when examining HIV risk among Latinas (CDC, 2017b). 


\section{Marianismo}

Marianismo can be described as the counterpart of machismo; it emphasizes the woman's role as a submissive partner who is oblivious about sex, but yet eager to sexually and emotionally please her male partner and must accept the machismo-driven infidelities of these partners. Latina mothers traditionally play a key role in the Latinx family (Gomez \& Marin, 1996). Latinasand especially Latina mothers—have traditionally been viewed as owing the family a duty of self-sacrifice (Galanti, 2003), such tradition is known as marianismo. Many Latina daughters adopt the marianismo tradition of sacrifice and its accompanying servitude, and keep in accordance with traditional cultural values. Daughters are often socialized to highly respect their mothers so they can be considered a buena hija (good daughter) (Villanueva Dixon, Graber, Brooks-Gunn, 2008).

Traditionally, Latinas are expected to be well behaved, obedient, pure, and mirror the innocence of the Virgin Mary of Christianity (the term marianismo derives from "Mary"). For instance, a Latina who engages in sexual intercourse before marriage would be considered a shame to the family honor and herself (Benavides, Bonazzo, \& Torres 2006). They are expected to remain virgins until marriage, then bear children, sacrifice, and commit themselves to husband and family (Gloria, Ruiz, \& Castillo, 2004; Upchurch, Aneshensel, Mudgal, \& McNeely, 2001). Such beliefs increase Latinas' risk and susceptibility for HIV and prescribe that Latinas should remain innocent and ignorant about sex and refrain 
from discussions about HIV risk; these beliefs also hinder condom use and negotiation skills (Marin et al., 1993; Moreno, 2007).

\section{Respeto}

Respeto is a traditional and core Latinx cultural value (Antshel, 2002; Cuellar, Arnold, \& Gonzalez, 1995; Freeman, Lewis, \& Colon, 2002; GonzalezRamos, Zayas, \& Cohen, 1998; Halgunseth, Ispa, \& Rudy, 2006; Marin \& Marin, 1991; Unger, Gallaher, Shakib, Ritt-Olson, Palmer, \& Johnson, 2002) that stresses obedience and consideration toward adults, parents, or elders by younger people (Delgado-Gaitan, 1994). It is "knowing the level of courtesy and decorum required in a given situation in relation to other people of a particular age, sex and social status" (Harwood, Miller, \& Irizarry, 1995, p. 98). In a 2010 study with Mexican and Dominican parents examining respeto in a Latinx parenting framework, one participant expressed the importance of children showing respeto to parents, grandparents, teachers, doctors, extended family members, and even strangers - particularly in public, where it is most important, as a matter of family honor. Another participant noted that respeto is optional "for the American" ... "but [not] for Hispanics" (Calzada, Fernandez, \& Cortes, 2010, p. 82).

Studies have shown that traditional cultural values influence attitudes and beliefs, which then influence health risk behaviors (Castro, et al., 2007; Gil, Wagner, \& Vega, 2000; Unger, Ritt-Olson, Teran, Huang, Hoffman, \& Palmer, 2002; Unger, Shakib, Gallaher, Ritt-Olson, Mouttapa, \& Palmer, 2006).

Specifically, respeto or respect should be shown to those who hold position of 
authority, elders, males, and those of high economic status; this can result in Latinx patients not questioning physicians or expressing dissatisfaction with services (Flores, 2000), making respeto a protective as well as potentially risky cultural belief.

\section{Familismo}

The term familismo, coined in the 1940s, signifies commitment to family, family members, and family relationships; commitment to family comes before oneself (Heller, 1970). Understanding this term requires an understanding of how different groups define family and the commitments that come with it. For instance, Asian culture generally defines family as filial piety (Cowgill, 1986; Unger et al., 2002), where children have a duty to be respectful of their parents, elders, and ancestors (Ng, Phillips, \& Lee, 2002). Yet, non-Latinx Whites generally define family in terms of the nuclear family and encourage individuality and independence (Cowgill, 1986; Unger et al., 2002). However, for Latinxs, family means the nuclear, extended, and fictive family (neither by blood or marriage), and loyalty to the family is encouraged and its members are raised to depend on family (Sabogal, Marín, Otero-Sabogal, Marín, \& Perez-Stable, 1987); this is familismo.

Research has consistently shown that Latinxs score higher in familismo when compared to non-Latinx Whites (Ferrari, 2002) or African Americans (Ramirez, Crano, Quist, Burgoon, Alvaro, \& Grandpre, 2004). In fact, irrespective of language preference or Latinx subgroup, familismo is a core cultural value for Latinxs (Villarreal, Blozis, \& Widaman, 2005). As such, it is a protective factor for 
drug use and risky behaviors (Gil, Wagner, \& Vega, 2000; Ramirez et al., 2004; Unger, Ritt-Olson et al., 2002) such as marijuana (Ramirez et al., 2004) and tobacco use (Kaplan, Napoles-Springer, Stewart, \& Perez-Stable, 2001). Familismo and respeto-after controlling for age, gender, socioeconomic status (SES), adult modeling (mirroring adult behaviors), peer influences, and acculturation—have both been found to be positively associated with substance use, including alcohol, which are risk factors for HIV infection (Kalichman, Simbayi, Kaufman, Cain, \& Jooste, 2007; Schensul, Singh, Gupta, Bryant, \& Verma, 2010; Shuper, Joharchi, Irving, \& Rehm, 2009; Shuper, Neuman, Kanteres, Baliunas, Joharchi, \& Rehm, 2010).

\section{Religion}

Past research on the sexual behaviors of Latinas have shown acculturation and religiosity to be determinants of risky sexual behaviors (Cuellar, Arnold, \& Maldonado, 1995). When HIV first emerged, religion was thought of as a barrier to prevention because some religions believed AIDS to be a punishment from God for the sins of practicing risky sexual behaviors. Additionally, some religions were against the promotion of condom use, since it was seen as promoting promiscuity (Balogun, 2010; Francesca, 2002; Monshipouri, \& Trapp, 2012). However, religion has shown to be mostly protective for Latinas against risky sexual behaviors (Abraido-Laza, Armbrister, Florez, \& Aguirre, 2006; Hill \& Pargament, 2003; Hunt, Schneider, \& Comer, 2004; Steensland et al., 2000). In fact, higher levels of religiosity are associated with lower levels of risky sexual behaviors, fewer lifetime sex partners, less anarchic attitudes, and later sexual 
debut (Edwards, Fehring, Jarrett, \& Haglund, 2008; Elifson, Klein, \& Sterk, 2003;

Liebowitz, Castellano, \& Cuellar, 1999; Nonnemaker, McNeely, \& Blum, 2003).

Yet, few studies have investigated the role of religion in the risky sexual behaviors of Latinas.

Although the aforementioned constructs have been shown in the literature to be relevant factors for exposure to HIV, not all relevant constructs will be found in the present study's dataset, since the data from the parent study were collected to examine attachments as a moderator to substance use among Latina mothers and daughters. However, the following relevant constructs were available in the parent data and will be included in the present study's model.

\section{Sociodemographic Variables}

\section{Socioeconomic status/income.}

Researchers have documented that annual household income, poverty level, and employment are financial factors significantly associated with HIV prevalence (De La Rosa, 2002; Krueger, Wood, Diehr, \& Maxwell, 1990;

UNAIDS, 2008; Riley, Gandhi, Hare, Cohen, \& Hwang, 2007). Moreover, the Center for Disease Control and Prevention reported that poverty drives ethnic disparities in HIV prevalence (as cited in CDC, 2015c). Additionally, women with low income are more likely to have an HIV infected partner (Ickovics et al., 2002), more likely to engage in commercial sex work (Bene \& Merten, 2008; Jarama, Belgrave, Bradford, Young, \& Honnold, 2007), and less likely to negotiate safe sex practices such as condom use (Henao-Martinez \& Castill-Mancilla, 2013; Salabarria-Pena, Lee, Montgomery, Hopp, \& Muralles, 2003). With $40 \%$ of 
Latinas living in poverty, compared to $10 \%$ for $n$-Latina White (as cited in CDC, 2015c), Latinas are left disproportionately vulnerable to HIV infection.

Prevalence of HIV is higher among individuals with low socioeconomic status (CDC, 2017d); specifically, SES factors such as low income and low education level (CDC, 2017d; Raphael, 2004, World Health Organization [WHO], 2010a) have been most commonly used in past research and will be examined in the present study. Compared to non-Latinx Whites, Latinxs have less income and less education—factors that are significantly exacerbated among foreign born immigrants (Gonzalez, Hendriksen, Collins, Duran, \& Safren, 2009). Women who report lower education and low income have a tougher time with condom and safe sex negotiations with their sex partners (Henao-Martinez \& Castill-Mancilla, 2013; Salabarria-Pena, et al., 2003), which is significant given that heterosexual contact has been shown to be the primary $(90 \%)$ mode of transmission for Latinas (CDC, 2017a).

\section{Nativity/documentation status.}

Timely diagnosis of HIV is vital in order to ensure survival and quality of life for HIV infected individuals (HIV-Causal Collaboration, 2010). However, undocumented immigrants are less likely to seek health care, including health screening, because of fear of the authorities and deportation (Asch, Leake, \& Gelberg, 1994), because translations services are often inadequate, and the difficulty meeting all paperwork requirements (Dang, Giordano, \& Kim, 2012); subsequently preventing immigrants from seeking medical care because of uncertainty in the system (Berk \& Schur, 2001; Bustamante et al., 2012). 
Additionally, undocumented status is a strong predictor of being medically uninsured (Bustamante, Chen, Fang, Rizzo, \& Ortega, 2013) and precludes immigrants from benefiting from the Patient Protection and Affordable Care Act (Obamacare) (Zuckerman, Waidmann, \& Lawton, 2011). Uninsured individuals are less likely to have regular medical checkups and, therefore, are less likely to receive preventive care; these individuals sometimes postpone medical attention, even when seriously ill (Ayanian et al., 2000). Yet, visiting a doctor in the past 12 months has been significantly associated with being tested for HIV (Van Handel et al., 2015).

\section{Education.}

Individuals with higher education levels are more likely to live longer and healthier and are more likely to practice preventative behaviors (Braverman \& Egerter, 2008; Cutler \& Lleras-Muney, 2010). Lower education levels have resulted in lack of awareness and knowledge about HIV prevention, which have been significantly associated with lower rates of HIV testing (Idris, Elsamani, \& Elnasri, 2015; Rios-Ellis et al., 2010; Ugarte-Gil et al., 2013). Additionally, Zambrana and collegues found that most Latinas $(60 \%)$ do not receive sex education from their parents and $21 \%$ reported not receiving sex education at school (Zambrana, Cornelius, Boykin, \& Lopez, 2004). Yet, Latinas that did report receiving sex education were more likely to have partners that consistently used condoms (Zambrana et al., 2004). In 2010, 64.4\% of Latinxs reported education level of high school diploma or above, compared to $88.2 \%$ of nonLatina White women; only $15 \%$ of Latinas reaching college level compared to 
$30 \%$ of non-Latina White women (US Census Bureau, 2016). Such estimates have placed Latinas at an educational disadvantage in regard to HIV awareness, knowledge, and prevention. Although education and income are closely correlated, in the current sample, they are not directly associated as many of these women may be highly educated in their native countries but do not speak English; hence, the women were unable to obtain high paying positions despite formal education.

\section{Sex under the Influence of Alcohol and other Drugs}

A large body of research has linked alcohol use to increased sexual risk behaviors (De La Rosa, Dillon, Rojas, Schwartz, \& Duan, 2010). Latinas report higher rates of sex under the influence of drug or alcohol when compared to nonLatina White or Black women (Adimora, Schoenbach, Taylor, Khan, \& Schwartz, 2011) and have a higher rate of acquiring HIV through heterosexual contact than that on non-Latina Whites (CDC, 2017b). A study by Baliunas and colleagues (2010) found the risk of HIV infection among individuals who report drinking alcohol is $77 \%$ higher than those who do not drink at all. Consuming alcohol specifically before or during sexual relations has been associated with an $87 \%$ increased risk of HIV infection compared to those who do not drink alcohol before or during sex (Baliunas, Rehm, Irving, \& Shuper, 2010). Moreover, those who engage in binge drinking are twice as likely to report engaging in HIV sexual risk behaviors compared to non-binge drinkers (Baliunas et al., 2010). Subsequently, the HIV rate among Latina women is over 4 times that of non-Latina White women. 


\section{Condom Use}

The CDC reported that the use of condoms is highly effective in the prevention of HIV (CDC, 2013). However, researchers have found that what dictates the use of condoms among sexual partners is not the effectiveness in the prevention of HIV, but the sexual relationship power that a woman may have in her relationship (Pulerwitz, Amaro, Jong, Gortmaker, \& Rudd, 2002). Yet among Latinxs, culture can dictate that condoms not be used with wives or in intimate relationships, because sex without condom is an expression of love (Catania et al., 1992; Marin, Tschann, Gomez, \& Gregorich, 1998). For men, irrespective of marital status, machismo encourages them to have multiple sex partners. Machismo is associated with low condom use and high risk for HIV infection for both the men and their partners (Beaulaurier, Craig \& De La Rosa, 2009; Cianelli, Ferrer, \& McEImurry, 2008; Pleck, Sonenstein, \& Ku, 1993). Women who report high marianismo beliefs are expected to behave, be inexperienced with sex, and not discuss sex, even though discussions about sex with partners is positively associated with condom use in this population (Warren, Harvey, \& Bovbjerg, 2011), Latinas continue to be infected via heterosexual sex with their intimate partners (CDC, 2017b). Yet, Latinas who carry condoms and insist on using them are perceived as promiscuous and not desirable as marriage partners (Weeks, Schensul, Williams, Singer, \& Grier, 1995). For Latinas, trying to maintain cultural and traditional values prevent them from questioning their husbands' extramarital affairs, negotiating condom use with their partners, and 
having open discussions about sex and risk behaviors (Hines \& Caetano, 1998;

Raffaelli \& Suarez-Al-Adam, 1998; Rojas-Guyler, Ellis, \& Sanders, 2005).

Although many Latinxs are aware of HIV risks and the advantages of condom use, they continue to report low condom use (Carballo-Diegues, Dolezal, 1996; Harvey \& Henderson, 2006; Knipper et al., 2007). Moreover, despite most HIV infections occurring in married and primary relationships (CDC, 2017b), Latinx couples report even lower instances of condom use with their married or primary partners (Pulerwitz et al., 2002).

\section{Communicating with Older Patient / HIV Counseling}

Aside from the lack of HIV awareness and knowledge among older adults, there is also a misconception in public health and the community: older adults are categorized as a low risk group—excluded from being at risk for HIV infection (Linsk, 2000; Adekeye et al., 2012). Not only do physicians have relatively little knowledge of HIVIAIDS in older adults, but they also experience discomfort when initiating discussions with this population about HIV (Hughes, 2011). In fact, a survey of 3000 US adults age 57 to 85 showed that only $22 \%$ of women and $38 \%$ of men reported discussing sex with their primary care physicians after age 50 (Lindau et al., 2007). Since some symptoms of HIV resemble signs of aging (e.g., fatigue, weight loss, mental confusion, and dementia) (Harris, 2015), physicians are disregarding the HIV infection as a possible diagnosis for their older patients, which consequently creates a disproportionate number of late stage diagnoses (within 12 months of AIDS diagnosis) among older adults (Mugavero et al., 2007). In a study of 113 patient records evaluated from Duke University, Sanders 
and her colleagues found that late diagnosis was most common among older patients. Late diagnosis leads to increased co-morbidity, mortality, spread of the virus, lower responsiveness to HAART, higher health care costs, and ultimately diminished quality of life (Sanders, Bayoumi, Holodniy, \& Owens, 2008). In fact, once diagnosed with HIV, the average life expectancy of persons over age 50 is 7 years, compared to 12 years among those age 13-49 (Inungu, Mokotoff, \& Kent, 2001; Lekas, Schrimshaw, \& Siegel, 2005; Nguyen \& Holodniy, 2008).

Several studies on mammogram testing have also shown that physicians are a strong referent on Latinas' decision to perform health related tests (Pasick et al., 2009; Stewart, Rakowski, \& Pasick. 2009; Tolma, Batterton, Thompson, \& Engelman, 2012; Washington, Burke, Joseph, Guerra, Pasick, 2009). In a sample of adult Latinas, Craig et al. (2012) found that over half (57\%) of middle age and older Latinas reported that their physicians failed to endorse counseling on safe sex or HIV testing. Furthermore, middle-age and older Latinas who reported being tested for HIV did so in a community clinic. Results showed that they were less likely to be tested by their primary care physicians (Craig, Beaulaurier, Newman, De La Rosa, \& Brennan, 2012). Similarly a cross-sectional study with a sample of 55 older African American and White women age 58-93, Lindau et al. found that most women $(75 \%)$ felt providers should initiate conversations about sex, but only about half (55\%) reported having discussed sex with their physicians since age 60 (Lindau et al., 2006). Such communication gaps have been significantly associated with low prevention screening—including HIV testing (Ayanian et al., 2000; Lindau et al., 2006; Timmins, 2002). 
Not being able to communicate properly with providers, leads to lost opportunities for endorsing HIV testing and prevention for Latinxs. Physicians are missing opportunities for prevention, early diagnosis, and early treatment (Fisher, 2008; Liddicoat et al., 2004). Too often, communication with physicians is hindered by low English proficiency among Latinxs, which serves as an obstacle in the patient-provider relationship (Jacobs, Karavolos, Rathouz, Ferris, \& Powell, 2005).

\section{Acculturation}

Acculturation is a process initiated through the integration of two or more independent cultural systems and by which immigrants modify their behaviors, attitudes, and culture toward those of the host society. It is not simply a culturechange; it is a "fundamental part of migration-induced adaptations" (Rogler, Cortes, \& Malgady, 1991, p. 585). Moreover, acculturation has been continuously linked as a key factor to Latinx health behaviors and outcomes (Amaro \& De la Torre, 2002; Andreeva, Unger, Yaroch, Cockburn, Baezconde-Garbanati, Reynolds, 2009; Caetano, Vaeth, Ramisetty-Mikler, \& Rodriguez, 2009; De La Rosa, Vega, \& Radisch, 2002; Rojas-Guyler, Ellis, \& Sanders, 2005). However, acculturation can have both positive, as well as negative effects, on risk behaviors and health outcomes among Latinxs (Norris \& Ford, 1994; Romero, Arguelles, \& Rivero, 1993; Sanchez, Rice, Stein, Milburn, \& Rotheram-Borus, 2010). For example, low acculturation is associated with low rates of HIV testing, and less reported condom use, but also with engaging in less risky behaviors; in contrast, higher acculturation has been associated with higher rates of HIV 
testing and use of condoms, yet higher rates of risky behaviors, such as multiple sex partners and earlier age of sexual debut (Hunt, Schneider, \& Comer, 2004; Lopez-Quintero et al., 2016; Lopez-Quinter, Shtarkshall, \& Neumark, 2005; Marin \& Marin, 1992; Marin et al., 1993; Rojas-Guyler, Ellis, \& Sanders, 2005). Additionally, less acculturated Latinxs are more likely to experience barriers to revealing their HIV status (e.g. familismo and marianismo), and more likely to perceive stigma from their own communities (Rajabiun et al., 2008).

In a San Francisco study performed with a sample of 460 Latinxs, Marin and Marin found that acculturation status was positively associated with knowledge of HIV transmission (1990). In fact, after controlling for education, less acculturated Latinxs were more likely to report that HIV could be transmitted through casual contact (e.g., shaking hands or sharing eating utensils) (Marin \& Marin, 1991). More recent studies have shown positive correlations between acculturation and HIV risk behaviors among Latinxs, such as low levels of condom use and substance misuse (De La Rosa et al., 2016; Berger Cardoso, Ren, Swank, Sanchez, \& De La Rosa, 2016). Similar results have been found among higher acculturated Latina women who were more likely to be intravenous drug users and have multiple sex partners (Rojas-Guyler, Ellis, \& Sanders, 2005). Additionally, foreign born Latinas report fewer sexual partners and lower rates of sexually transmitted infections and alcohol or drug abuse, but are less likely to be tested for HIV or use condoms (Castillo-Mancilla, et al., 2012). In addition, acculturation is a factor in HIV stigma. For example, Florez et al. found that Latinxs with lower acculturation show significantly higher levels of drug use 
and drug addiction stigma (Florez et al., 2015). When using language as a proxy, researchers have shown that lower acculturated Latinxs are more likely to report stigma-related concerns that would impact HIV status, such as sexual orientation and drug use, and less likely to communicate their positive serostatus (Rajabiun et al., 2008).

\section{HIV Testing}

Efforts to increase HIV testing have emerged as a critical part of HIV prevention (US Department of Health \& Human Services [HHS], 2011); healthcare provider endorsements has been found to be the primary reason patients consent to HIV testing, particularly for older adults (Craig et al., 2012; Hillman, 2008; McMahon, Wanke, Terrin, Skinner, \& Knox, 2011). In fact, studies on mammogram testing have also shown that physicians are a strong referent on Latinas' decision to perform health related tests (Pasick et al., 2009; Stewart, Rakowski, \& Pasick. 2009; Tolma et al., 2011; Washington et al., 2009). Risk behaviors among people aware of their seropositive status are reduced therefore, making HIV testing a deterrent to the spread of HIV (Harrison, Song, \& Zhang, 2010; Mugavero et al., 2007; Infectious Diseases Society of America, 2008). Still, approximately $21 \%$ of HIV positive people in the US are unaware of their status. When compared to non-Latinx Whites and African Americans, Latinxs have higher rates of late HIV diagnosis and are less likely to adhere to their treatment plan (CDC, 2003; Harrison et al., 2010). Harrison et al. noted that although life expectancy after time of diagnosis has increased for Latinxs, HIV health disparities among Latinxs are still of concern to public health (2010). 
Efforts to increase HIV testing have emerged as a critical part of HIV prevention. The CDC has recommended the reduction of barriers to routine HIV tests. Routine testing has been recommended for all individuals, 13-64 years of age, in all health care settings, including primary health-care providers in the public and private sectors, hospital emergency departments, urgent care clinics, inpatient services, substance abuse clinics, public health clinics, community clinics, and correctional health-care facilities (HHS, 2011). According to the CDC (2017b), barriers to HIV testing can include stigma, lack of HIV knowledge and awareness, lack of health care providers endorsing HIV testing, and sociocultural and socioeconomic factors.

It is noteworthy that the CDC recommendations exclude patients age 65 and over from routine testing, unless the patient has risk factors for HIV infection (CDC, 2006). Similarly, physicians hold stereotypes about older adults, such as not being drug users or having multiple partners, and do not perceive them to be sexually active (Paltiel et al., 2005), even though researchers have shown that older adults remain sexually active well into their 80 s (Lindau et al., 2007). In a study conducted in New York, researchers found that from 1995 to $2005,41 \%$ to $13 \%$ respectively, of adults over 50 who were HIV positive were infected via intravenous drug use (IDU) (Karpiak, Shippy, \& Cantor, 2006). Studies on HIV among older people have suggested that factors such as risky sexual behaviors and IDU are also a threat to the 50 and over population to being infected with HIV. 
However, research has indicated that older Latinas in particular are less likely to speak to a health care provider about safe sex practices or HIV testing than their younger counterparts (Craig et al., 2012; Hillman, 2008; McMahon et al., 2011); this makes older Latinas particularly vulnerable to late diagnosis (Hughes, 2011), since researcher has shown that HIV testing is an important factor to HIV prevention (DiFranceisco, Pinkerton, Dyatlov, \& Swain, 2005; Marks, Crepaz, Senterfitt, \& Janssen, 2005). Nevertheless, older adults are generally not routinely tested for HIV (Linsk, 2000; Savasta, 2004).

\section{Attachment}

Attachment Theory, developed by John Bowlby, concerns the strong and ongoing emotional bond that connects one person (e.g., a caregiver, mother) to another (e.g., a child); this emotional bond is irrespective of time and space (Ainsworth, 1973; Bowlby, 1969). Bowlby's theory proposes that children are born biologically encoded to build attachment with their mothers as a survival mechanism (Bowlby, 1969). In 1935, Konrad Lorenz found that attachment was inborn in baby ducklings and thus a survival skill (Lorenz, 1935), this led to his Imprinting Theory, which later became influential to Bowlby's Attachment Theory.

Attachment Theory was first developed as a model to explain the early parent-child bond (Bowlby, 1969); research on attachment among adults has been increasing because it influences social functioning, well-being, stress, coping skills, morbidity, and health behaviors (Ciechanowski \& Katon, 2006;

Ditzen, et al., 2008; Maunder \& Hunter, 2001; Maunder, Lancee, Hunter, Greenberg, \& Steinhart; 2005; Maunder, Lancee, Nolan, Hunter, \& Tannenbaum, 
2006; Meredith, Strong, \& Feeney, 2006; Schmidt, Nachtigall, WuethrichMartone, \& Strauss, 2002; Waller, Scheidt, \& Hartmann, 2004). Attachment to family members has been characterized by mutual trust, communication, and closeness across all stages of development (Armsden \& Greenberg, 1987).

Among Latinas, attachment and family influence has been associated with good physical and mental health outcomes (Plant \& Sachs-Ericsson, 2004). Yet, the vast majority of studies of protective familial influences on Latinas have involved adolescent samples (Robles et al., 2006; Romo et al., 2001). However, more recent studies on adult Latina mothers and daughters have suggested that attachment may play a protective role in substance use (De La Rosa et al., 2015), risk behaviors that may lead to HIV (De La Rosa et al., 2010a,) and overall health (Washington, 2009).

Traditional cultural values influence the life long bonds that Latina mothers and daughters often forge-in which they rely heavily on each other for support over the lifespan (Dixon, Graber, \& Brooks-Gunn, 2008). Recent research has explored that bond among adult Latinas and their mothers. In fact, addressing attachment among adult Latina mother-daughter dyads is being examined for the implementation of risky health behavior preventions for older Latinas (Browne \& Chan, 2012; Kratzke, Amatya, \& Vilchis, 2014; Sinicrope et al., 2009). As such, adult Latina daughters may be an untapped source to overcome the barriers that currently keep older Latinas from accessing health related knowledge and practicing risk prevention (Washington et al., 2009). 


\section{Daughter-Initiated Communication on Health-Related Behaviors}

Studies have indicated a one-directional transfer of health-related influence and information from mother to daughter (Hutchinson, Jemmott, Jemmott, Braverman, \& Fong, 2003; Kratzke, Vilchis, \& Amatya, 2013; Romo, Bravo, \& Tschann, 2014; Santa Maria, Markham, Bluethmann, \& Mullen, 2015;

Widman, Choukas-Bradley, Noar, Nesi, \& Garrett, 2016; Wight, Fullerton, 2013). However, recent studies have suggested that daughters may be uniquely positioned to influence their mothers' health-related behaviors and decisions (Browne \& Chan, 2012; Kratzke, Amatya, \& Vilchis, 2014; Mosavel, 2009; Mosavel, \& Genderson, 2016; Mosavel, \& Thomas, 2009; Washington et al., 2009). Adult daughters have been shown to be a source of support and assistance in health-related issues for their aging mothers, due in part to their better ability to access and navigate the health care system, higher education, and greater familiarity with health-related terminologies (Jacobs et al., 2005; Tolma et al., 2012; McGraw \& Walker, 2004; Washington et al., 2009). Additionally, older Latina mothers depend on their more acculturated daughters to bridge language gaps between them and health care providers and healthrelated information (Kratzke, Amatya, \& Vilchis, 2014; Washington et al., 2009).

In a 2014 mixed-methods study on breast cancer information-seeking among young college women, $18 \%$ of adult daughters reported passing the breast cancer information they learned to their mothers (Kratzke, Amatya, \& Vilchis, 2014). Approximately half (49.3\%) of the study participants where Latinas, all were college students, and $35 \%$ had a family history of breast cancer. 
Factors significantly associated with sharing breast cancer information with mothers were: university level of education (OR 1.31), practicing breast selfexams (BSF) (OR 2.38), and actively and passively obtaining information online (OR 1.89 and OR 2.95, respectively) (Kratzke, Amatya, \& Vilchis, 2014). Qualitative analysis revealed that risk factor knowledge and age appropriate testing were the themes that emerged most with daughters who shared information. Most of the daughters reported influencing their mothers to focus on their adherence to testing, such as mammograms, clinical breast exams, and BSFs (Kratzke, Amatya, \& Vilchis, 2014).

A study by Washington et al. investigated the influence that adult daughters have on their mothers' health behaviors and found that adult daughters play a significant role in their mothers' choice of health care providers as well as the quality of care they receive. Furthermore, the daughters were found to motivate their aging mothers in seeking out a health care provider (Washington et al., 2009). Mothers who reported higher levels of attachment and emotional ties to their daughters were more likely to follow their daughters' health-related advice (Washington et al., 2009).

The studies noted above have suggested adult daughters may be influencing their mothers' health-related behaviors-specifically in the context of cancer screenings (Browne \& Chan, 2012; Kratzke, Amatya, \& Vilchis, 2014; Sinicrope et al., 2009; Washington et al., 2009). However, to date, no study has investigated the influences that adult Latina daughters may have on their mothers' risky sexual behaviors and HIV testing. 
The present study aimed to begin addressing this gap in the research literature by exploring the mother-daughter relationship interaction on the associations between adult Latinas' risky sexual behaviors and their older mothers' risky sexual behaviors. Similar to previous adult mother-daughter health studies, the present study hypothesized that an association would exist between the daughter's risk behaviors and her mother's risk behaviors and influenced by the dyadic relationship.

\section{Theoretical Framework}

The present study was guided by Social Cognitive Theory (SCT). Developed by Albert Bandura in the mid-1980s (Bandura, 2001), SCT explains how people attain and maintain certain behavioral patterns (Bandura, 1997). In the beginning, SCT was a theoretical derivative of Millard and Dollard's Social Learning Theory (SLT) (1941). In 1963, Bandura and Walters expanded SLT_adding principles of observation, or vicarious learning (Bandura \& Walters, 1963). Then, in 1977, Bandura introduced self-efficacy to the model, and renamed it Social Cognitive Theory (Edberg, 2015). Bandura found that people learn by observing the behaviors and attitudes of others, and outcomes of these behaviors (1977). The key factors that are involved in SCT are environment, the individual, and the behavior; these factors influence each other-no single factor is a simple result of the other (Glanz, Rimer, \& Lewis, 2002). Environments, whether physical (temperature or size of a room), social (family, friends, colleagues), represent factors that can affect or influence the person's behavior. The individual is the person being influenced and the behavior that may be 
influenced. The mutual interaction and influence that the individual, behavior, and environment have on each other is what Bandura called reciprocal determinism.

In the model, the adult daughter represents the environment (family, friends, and colleagues) that influences the older mother (behavior). The risk behaviors of the adult daughter (environment) also influences the mother's risk behaviors (behaviors). When daughters share information they have via education or HIV Counseling (personal attitudes), again this influences the mother's risk behaviors. These fluid interactions, influence the risk behaviors of the older mothers.

However, Parents and other family members significantly influence patterns of development in the individual (Perrino, Gonzalez-Soldevilla, Pantin, \& Szapocznik, 2000). These influences extend well beyond childhood and adolescence, such that family influences continue to be important into adulthood (Fergusson, Boden, \& Horwood, 2007; Overbeek, Stattin, Vermulst, Ha, \& Engels, 2007). For instance, adult children and their parents-especially mothers and daughters, have been shown to influence one another's behavior considerably throughout the lifespan (Fingerman, Chen, Hay, Cichy, \& Lefkowitz, 2006). Studies have shown mother-daughter communication relationships influence health behaviors such as breast. Therefore, although not part of the Bandura's Social Cognitive Theory (SCT), the present study proposes that the level of the adult mother-daughter relationship or their attachment will interact (or moderate) the influence that the environment (or adult daughter) may have over the older mother's risk behaviors. 
Like the Bandura's SCT, this study explores the behaviors between the adult Latina daughters and that of their older mothers' risky sexual behaviors. Furthermore, the study explores the mother-daughter relationship's effect on associations between adult Latinas' risky sexual behaviors and that of their older mothers' risky sexual behaviors. The theoretical model for the present study is presented in Figure 1.

\section{Summary of Present Study}

The present study was conducted using secondary data and aimed to investigate the associations that may exist between the risky sexual behaviors of adult Latina daughters and the risky sexual behaviors of their older Latina mothers, and examine whether the mother-daughter attachment patterns moderate existing associations of risky sexual behaviors.

This investigation evaluated all constructs at every time point. For the purpose of this study, alcohol users will be defined in terms of at least one binge drinking episode per month (adapted from Naimi et al., 2003), at least 4-5 glasses of wine, 3-4 cans/bottles of beer, or 3-4 four-ounce drinks of hard liquor per occasion during the 12 months prior to assessment. The National Institute on Alcohol Abuse and Alcoholism defines moderate drinking for women as having up to 1 drink per day; heavy drinking is defined as 5 or more drinks on the same occasion on 5 or more days in the past 30 days; and binge drinking is defined as 4 drinks within 2 hours or 5 drinks on the same occasion at least once in the past 30 days (National Institute on Alcohol Abuse and Alcoholism [NIAAA], n.d.). 
The overall objective of the present study was to determine (a) the influence that adult Latina daughters' recurrent HIV risk behaviors have on those of their older Latina mothers, (b) and how mother-daughter attachment moderates those associations. The study research model is presented in Figure 2. The study focused on the association of factors that place older Latinas at high risk for HIV such as sexual risk behaviors, lack of HIV testing, and HIV counseling or knowledge, as well as how relationships with their adult daughters may interact with the associations.

The present study addresses the gap in the literature examining sociocultural aspects of HIV risk infection among older ethnic/racial minority women; its public health impact includes providing evidence for the need to create culturally tailored programs for the growing ethnic/racial minority population that is living longer due to advances in medical treatment. Such knowledge is necessary to facilitate the development of age and culturally appropriate prevention and intervention strategies that can ameliorate the disproportionate impact of HIV among older Latinas. Results from the proposed study would help in developing interventions aimed at preventing HIV among this growing population.

Furthermore, findings from the present study could also enhance existing programs that use one-directional intervention from mother to daughter-where mothers are influencing the decisions of their adolescent daughters. Instead, results can provide a basis for an alternative direction or bi-directional approach 
to HIV prevention among older Latinas, where adult daughters may be the independent variable that influences the health decisions of their older mothers. Innovation

Unlike most studies on protective familial influences on Latina substance use and HIV risk behavior (Robles et al., 2006; Romo et al., 2002), the present study innovatively focused on older Latinas-a population that is understudied in terms of its HIV risk behaviors. Moreover, the study examined a sample of Latina mother-daughter dyads. Moreover, most Latinx studies have focused primarily on Mexican/Chicano populations; however, data obtained for the parent study was collected in Miami-Dade, Florida that provides a rich and diverse Latinx community with populations from all over Latin America and the Caribbean. In addition to the innovation of the sample, mother-daughter attachment is being examined as a potential moderator. This is the first investigation to longitudinally examine the relationship of recurrent HIV risk behaviors of adult Latina daughters to that of their older Latina mothers. The study highlighted the importance of adult Latina mother-daughter relations in its examination of these recurrent behaviors.

\section{Research Aims}

Aim 1: Examine the association between recurrent HIV sexual risk behaviors of adult Latina daughters and those of their older Latina mothers.

Hypothesis: Recurrent HIV risk behaviors among adult Latina daughters will be positively associated with recurrent HIV risk behaviors among older Latina mothers. 
Aim 2: Examine the moderation effect that the mother-daughter attachment moderator may have on the associations between the recurrent HIV risk behaviors of adult Latina daughters and those of their older Latina mothers.

Hypothesis 1: Mothers with higher attachment levels to daughters will show stronger association between recurrent HIV risk behaviors of adult Latina daughters and those of their older Latina mothers.

Hypothesis 2: Mothers with lower attachment levels to daughters will show weaker association between recurrent HIV risk behaviors of adult Latina daughters and those of their older Latina mothers.

Statistical analysis for the current study was completed using Statistic Package for the Social Sciences, version 24 (SPSS v24) software (Field, 2013) and the Hayes PROCESS modeling macro tool for SPSS. Descriptive statistics were performed on all variables in order to summarize data and profile all variables. Variables measured on a non-metric scale, such as categorical variables, were summarized as proportions; variables measured in metric scales, such as numerical variables, were summarized as means, median, mode, range, and standard deviations. 


\section{CHAPTER III. METHODS}

\section{Summary of Parent Study and Data Source}

This chapter will provide a detailed description of participants, sample recruitment, and data collection methods for the parent study as well as baseline and follow-ups, the use of measures, independent and dependent variables, and analytical approaches used in the research model. The present investigation analyzed baseline $\left(T_{0}\right)$ and follow ups $\left(T_{1}, T_{2}, T_{3}\right)$ using recurrent cross-sectional analysis (Grossoehme \& Lipstein, 2016), the study examined the following constructs across all four time points: (a) HIV sexual risk behaviors in the past 12 months, including sex under the influence of alcohol, multiple sex partners, and sex without a condom, (b) HIV testing and, (c), HIV counseling by a health care provider, and (c) the influence of mother-daughter attachment.

HIV sexual risk behaviors is the composite score of sex under the influence of alcohol, multiple sex partners, and sex without the use of a condom. Although HIV testing and HIV counseling indicate preventative practices, not practicing such behaviors would, in contrast, indicate a risk. Lastly, ttachment is composed of three subscales: alienation, communication, and trust, with a total of 25 indicators. The three subscales of all indicators are presented in Figure 2.

Data were obtained from the parent study: A Longitudinal Study of Substance Abuse and HIV Risk among Adult Latina Mother-Daughter Dyads (hereafter, the Women Study). The Women Study is a National Institute of Health (NIH)-funded 7-year assessment longitudinal cohort study conducted in Miami, Florida, and approved by the Florida International University (FIU) institutional 
review board (R01 NR012150-01). The Women Study Principal Investigator is Dr. Mario De La Rosa, director of Center for Research on U.S. Latino HIVIAIDS and Drug Abuse (CRUSADA). The overall goal of the parent study was to reduce the rate of incidence of health disparities related to HIVIAIDS and substance abuse among adult Latinas. The specific aims of the Women Study were threefold:

Aim 1: Determine the influence of social determinants on trajectories of change for substance use and HIV risk behaviors in a community-based sample of adult Latina mother and daughter dyads.

Aim 2: Determine the influence of mother-daughter attachment on trajectories of change for substance use and HIV risk behaviors in a community-based sample of adult Latina mother and daughter dyads.

Aim 3: Determine the moderating role of adult Latina mother-daughter attachment on associations between experiences of detrimental social determinants and trajectories of change for substance use and HIV risk behaviors in a community-based sample of adult Latina mother and daughter dyads.

The central hypothesis of the parent study was that social determinants, moderated by adult mother-daughter attachment, would influence substance use and HIV risk behavior trajectories in a community-based sample of adult Latina mother and daughter dyads. The Women Study design included 4 waves of data collection. Baseline $\left(\mathrm{T}_{0}\right)$ data were from a cross-sectional pilot study of intergenerational drug use and HIV risk behaviors among adult Latina mother 
and daughter dyads, funded by the National Institute on Drug Abuse (R24DA014260, PI: Mario De La Rosa). The subsequent three waves ( $\left.T_{1}, T_{2}, T_{3}\right)$ consisted of a 5-year post-baseline follow-up ( $\left.T_{1}\right)$ and two waves $\left(T_{2}, T_{3}\right)$ that were conducted 1 year apart from each other. The parent study longitudinal timeframe is presented in Figure 3.

\section{Baseline Data $\left(T_{0}\right)$ \\ Recruitment.}

Recruitment for baseline participants included announcements at local venues such as community health fairs, health clinics, drug court programs, Alcoholics Anonymous (AA) and Narcotics Anonymous (NA) meetings, Spanish radio stations and television channels, and a local newspaper. A variety of sources were used to maximize the likelihood that we would be able to recruit both substance using and non-using participants. Inclusion criteria were: (a) consenting to be interviewed for at least 2-3 hours; (b) being 18 years of age or older; (c) self-identifying as Latina; (c) living in Miami-Dade County, Florida; (d) being able to participate as a mother-daughter dyad; and, (e) willingness to provide two telephone numbers to researchers for correspondence during participation. The baseline data included standardized measures of attachment, alcohol use, and HIV risk behaviors, along with a variety of demographic variables. Before being considered for the study, potential participants were screened via phone for eligibility. Inclusion criteria for the baseline and follow-ups are presented in Table 1. 


\section{Data collection.}

Data collection procedures consisted of facilitated paper/pencil structured questionnaires at baseline. Face-to-face interviews were conducted in either

English or Spanish—based on the preference of each participant—and completed by trained bi-lingual (English/Spanish) interviewers at a location agreed upon by both the interviewer and participants. Interviews lasted approximately 2-3 hours and were usually administered in the participants' homes; participants were compensated for their time with $\$ 40$ cash upon completion of interview. Once collected, data were entered into a Microsoft Access database in the language in which the data was collected. Subsequently, data were exported to Software Package for the Social Science (SPSS v. 14) as string values and converted to numerical values using syntax created from a codebook for analysis.

\section{Participants/sample.}

Baseline data were collected on a community-based sample of 158 adult Latina mother-daughter dyads $(N=316)$ living in the Miami-Dade area. Recruitment strategies included advertising in Craigslist, radio announcements, and flyers. Dyads were stratified across four substance use categories: Dyad $1=$ substance-using mothers and substance-using daughters $(n=80)$; Dyad $2=$ substance-using mothers and non-using daughters $(n=38)$; Dyad $3=$ non-using mothers and substance-using daughters $(n=102)$; and Dyad $4=$ non-using mothers and non-using daughters $(n=96)$. Classification of mothers and daughters into the four dyads was based on whether they qualified as users of 
either alcohol, marijuana, cocaine, heroin, ecstasy, and/or prescription drugs. Every effort was made to maintain a diverse sample, therefore, respondent driven sampling (RDS) was used as the primary strategy for recruitment into the parent study.

The RDS technique was developed as a response to the judgement that chain-referrals were seen as convenience sampling (Heckathorn, 2011). RDS was established during an AIDS prevention intervention that targeted active drug users for interviews, for AIDS prevention, and HIV testing and counseling (Broadhead \& Heckathorn, 1994; Broadhead, Heckathorn, Grund, Stern, \& Anthony, 1995; Heckathorn \& Broadhead, 1996). This technique is an effective strategy in recruiting participants from hidden populations such as substance users and groups at risk for HIV infection (Heckthorn, 1997). "RDS produces samples that are independent of the initial subjects from which sampling begins. As a result, it does not matter whether the initial sample is drawn randomly." (Heckathorn, 1997, pp. 176-177).

Sixty-six percent of the overall sample reported being born outside of the United States. Most of the mothers (84.2\%) had emigrated to the United States, and less than half of the daughters (46\%) were US born. In terms of overall sample composition, $23 \%$ of the participants reported being Cuban, $14 \%$ Colombian, 5.4\% Peruvian, 4.4\% Dominican (Republic), 4\% Nicaraguan, 3.8\% Honduran, 3.8\% from Puerto Rican, and approximately $11 \%$ from other LatinAmerican countries, predominantly South American countries. About $29 \%$ reported less than high school education, $25 \%$ GED or high school diploma, and 
$47 \%$ beyond high school education. At baseline, the average participant age was 52.2 years $(S D=9.81$, range of 33 to 88 years) for mothers, and 27.6 years ( $S D$ $=8.96$, range of 18 to 54 years) for daughters. Additionally, half the participants reported personal incomes of less than $\$ 10,000$ per year.

\section{Follow-ups $\left(T_{1}, T_{2}\right.$, and $\left.T_{3}\right)$}

The first follow-up $\left(T_{1}\right)$ was conducted 5 years post-baseline and two subsequent follow-ups ( $T_{2}$, and $\left.T_{3}\right)$ were completed 1 year apart and concluded by Spring of 2014 with a final retention rate of $91 \%(n=286)$ at $T_{3}$. Because participants were being retraced for follow-ups, tracing and retention strategies differed slightly; with the development of new interviewing tools, methods for data collection also differed for follow-ups. Follow-up retention and data collection methods are described below.

\section{Locating participants (recruitment).}

A full-time community outreach worker, six interviewers (that previously worked in the baseline study), and a graduate student contacted participants by phone or in person by visiting address on file. Study personnel used several relocation methods in order to locate participants. Initially, the community worker would (1) refer to the screening form used during baseline, which usually contained phone numbers and an address for the participant. In the event the participant could not be reached by phone, personnel would (2) attempt to contact the person who referred the participant to the baseline study, whose information was obtained from the participant during a data collection point. If neither attempt was successful, an outreach worker would (3) visit the address 
listed for the participant on the screening form. After the contact information from the screening form was exhausted, (4) a trained graduate student researched the participant location via LexisNexis, a professional software tool used by academic institutes, government agencies, law enforcement, and private companies that perform tasks such as legal research, investigations, and identity searches. LexisNexis provided information necessary to help track participants who proved to be hard-to-reach. Information included updated phone numbers, addresses, and married surnames of participants, phone numbers and addresses of neighbors, friends, relatives, and reports of deceased participants.

\section{Data collection.}

Although baseline data were collected using paper/pencil structured questionnaires, for the follow-up interviews, the same measures were used and a similar questionnaire was designed using Questionnaire Development System 2.6 (QDS). Using QDS, questionnaires were developed as computer assisted personal interviews (CAPI). Subsequently, data collection procedures for all follow-ups $\left(T_{1}, T_{2}\right.$, and $\left.T_{3}\right)$ consisted of face-to-face interviews via CAPI. Contact information was collected in order to maintain communication with participant throughout the course of the remaining data collection waves. If a participant provided consent, interviews were also audio recorded for purposes of quality control. Afterward, data were uploaded into appropriate statistical software (SPSS/Mplus) packages for cleaning and analysis. Interviews continued to be conducted in either English or Spanish, as preferred by the participant, and completed by trained bi-lingual (English/Spanish) interviewers at a location 
agreed upon by both the interviewers and participants. Interviews lasted approximately $1-1 \frac{1}{2}$ hours and participants were given a monetary compensation of $\$ 50$ for their time.

\section{Present Study Data Source}

The present study sample consisted of 79 adult Latina mother-daughter dyads ( $N=158)$ from longitudinal secondary data obtained from the aforementioned (parent) Women Study. Mothers who were age 50 and over at the time of baseline were chosen for analysis, along with their corresponding adult daughters. The age of 50 and over was established by the World Health Organization as the definition for older adult for an aging project (WHO, 2010b). The same age was used in the current study in order to maximize sample size for analysis. Only dyads that participated in all four timepoints $\left(T_{0}, T_{1}, T_{2}\right.$, and $\left.T_{3}\right)$ were included in the analysis.

\section{Present participants/sample.}

Overall, most of the sample reported being born outside of the United States (Table 2 show countries of origin), and over half reported less than high school education. At baseline, the average participant age was 58.5 years for mothers, and 31.3 years for daughters. Overall, more than half the participants reported personal incomes of less than $\$ 10,000$ per year with the majority making less than $\$ 30,000$ per year, Table 3 shows individual descriptive statistics for mothers and daughters. 


\section{Measures.}

Data collection and measurement tools were selected from the parent study for their relevance to the present dissertation. All the selected measures were standardized scales with adequate psychometric properties. The variables and measures for all four waves included in the present study are described below.

\section{Inventory of parent and peer attachment (ippa) (attachment).}

A 25-item version of the IPPA (Armsden \& Greenberg, 1987) was used to assess attachment between mothers and daughters. Originally, the IPPA was developed to measure young adults' and adolescents' perceptions of relationships (both positive and negative affective/cognitive dimensions) with their parents and close peers. The measure consists of three subscales (Quality of anger and alienation, communication, and mutual trust) rated on a 5-point Likert-type scale ranging from Almost never or Never true to Almost always or Always true. Participants were instructed to report their attachment with their mother/daughter by answering questions such as: "My (mother/daughter) helps me to understand myself better.", "If my (mother/daughter) knows something is bothering me, she asks me about it."

The IPPA has demonstrated acceptable internal consistency and testretest reliability coefficients in previous research (Adam \& Chase-Lansdale, 2002). Cronbach's alpha coefficients in a sample of college students age 16-20 ranged between 0.72 and 0.91 for the sub-scales across both the parent and peer scales (Armsden \& Greenberg, 1987). In a sample of 18-20 year-olds, test- 
retest reliability was reported with Cronbach's alpha coefficients ranging between 0.93 for parent attachment and 0.86 for peer attachment (Armsden \& Greenberg, 1987). Furthermore, numerous other studies have supported the reliability and validity of the IPPA with adolescent samples (Adam \& Chase-Lansdale, 2002; Black \& McCartney, 1997; Formoso, Gonzales, \& Aiken 2000; Ridenour, Greenberg, \& Cook, 2006).

Because the IPPA was originally developed for use with adolescents and young adults, a confirmatory factor analysis was conducted to assess factor structure with the adult sample in the parent study. The confirmatory analysis produced an inadequate fit to the data from the mother only, daughter only, and total samples. Chi-square values (272 df each) was $630.87(p<.001)$, comparative fit index (CFI) values was .89, and the value for root mean square error of approximation (RMSEA) was .07. Additionally, the alienation subscale yielded poor evidence of internal consistency (such as Cronbach's alpha coefficient of .68 with mother sample). As a result of the confirmatory analysis, a total attachment IPPA scale score was used for the parent study by first reverse scoring the alienation scale items and then calculating the sum of all 25 items including communication and trust. Cronbach's alpha coefficients for the total sample were 0.93 (0.93 for daughters; 0.92 for mothers) (De La Rosa et al., 2010b)

HIV testing. HIV testing was measured at $T_{0}$ by asking participants whether they had ever been tested for HIV; for each follow-up, participants were 
asked if they had been tested since the last interview. Responses were collected dichotomously $(1=Y e s, 2=N o)$.

\section{HIV counseling.}

HIV counseling was measured at $T_{0}$ and for each follow-up by asking if a doctor or health care professional had spoken to them about HIV prevention or safe sex in the last 12 months. Responses were collected dichotomously (1=Yes and $2=$ No).

\section{HIV risk behaviors.}

Due to small sample size, this variable was recoded by combining several variables. Variable includes having sex under the influence of alcohol, having vaginal sex without condoms, and having multiple sex partners (more than one within the last 12 months). If the participant met the criteria for being at risk for any one of the variables, she would be categorized as having HIV risk behavior. The final variable used in the analysis was binary: 1=identified risk behavior(s) and $0=$ no risk behaviors.

Sex under the Influence of Alcohol. Sexual behavior under the influence of alcohol was measured with three items taken from a survey from an investigation on substance use and youth risky sexual behavior trajectories (Life Course and Health Research Center, 1997). As in previously published research with the present sample (De La Rosa et al., 2010a), the three selected items were used to create a sex under the influence of substance risk index (SUISRI) for alcohol. The first item assessed how often the respondent (or her partner) drank alcohol before or during sex during the past 12 months. Participants were asked to 
indicate frequency on the following scale for each item:1=Never, 2=Rarely, 3=Sometimes, 4=Usually, 5=Always. The second item assessed, on average, how strongly the participant was affected (i.e., intoxicated) by alcohol use prior to or during sex. The third item asked how strongly the participant's sexual partner was affected (i.e., intoxicated) by alcohol use prior to or during sex. For these two items, participants were asked to indicate their response on a scale of $1=N o t$ at all, 2=Somewhat, or 3=Very Strongly. The sex under the influence of alcohol risk index (SUIARI) score was calculated by summing the three items.

Sex without condoms. Sex without condoms was measured by the frequency of vaginal or anal sex in the past 12 months minus the frequency of condom use during the times participants had sex. A number above zero would indicate having sex without condoms.

Having multiple sex partners. Participants were asked the number of sexual partners in the past 12 months. More than 1 sexual partner indicated multiple sex partners.

\section{Acculturation.}

The measure used for acculturation was the Multidimensional Measure of Cultural Identity for Latinos (MMCISL) (Felix-Ortiz, Newcomb, \& Myers, 1995). The original MMCISL is a 35 -item measure across ten scales-each measuring a different dimension of cultural identity. Dimensions include Spanish Language Proficiency, English Language Proficiency, Familiarity with American Culture, Familiarity with Latino Culture, Preferred Latino Affiliation, Perceived Discrimination, Respeto, Spanish Language Preference, Latino Activism, and 
Feminism. Because of low reliability with the baseline sample (i.e., Cronbach's alpha < 0.70) (Dillon, Félix-Ortiz, Rice, De La Rosa, Rojas, \& Duan, 2009), Perceived Discrimination, Respeto, Spanish Language Preference, Latinx activism, and Feminism were not used in the follow-ups ( $T_{1}, T_{2}$, and $\left.T_{3}\right)$. Consequently, the present study used the scales below.

(1) Spanish Language Proficiency and (2) English Language Proficiency items asked respondents to rate their agreement with each item on a four-point Likert-type scale $(1=$ not at all, $2=$ fair, $3=$ good, $4=$ excellent). Items from each scale asked how well the participant speaks, reads, and writes each language. Cronbach's alpha reliability estimates of .90 (mothers) and .91 (daughters) were found for the Spanish Language Proficiency scale and estimates of .94 (mothers) and .97 (daughters) were found for the English Language Proficiency scale.

(3) Familiarity with Latino Culture and (4) Familiarity with American Culture scales asked respondents to rate their agreement with each item on a four-point Likert-type scale $(1=$ I don't know anything about these, $2=$ I know very little about these, 3= I know something about these, 4= I know a lot about these). Items from each scale asked how familiar a participant was with (a) fine art and artists, (b) history and politics, and (c) cultural legends and symbols of their Latin American country and of the United States, respectively. Cronbach's alpha reliability estimates of .82 (mothers) and .79 (daughters) were found for the Familiarity with Latino Culture scale; and estimates of .78 (mothers) and .80 (daughters) were found for Familiarity with American Culture scale. The finaly 
variable used was the Spanish Language Proficiency as a proxy for acculturation.

\section{Sociodemographics.}

The present study used four dependent sociodemographic variables to include age, marital status, socioeconomic status, and nativity.

Age. Participants were asked for date of birth at baseline. All other time points used the same date in order to calculate age at time of interview. Data were analyzed on number of years.

Marital status. Participants were asked during each interview about their marital status. Nominal information was collected to include: 1= Married, $2=$ Remarried, 3= Widowed, 4=Separated, 5= Divorced, $6=$ Never married, $7=$ Living with partner. Marital status was then recoded as a binary variable: $1=$ Married, Remarried, Living with Partner and 2= Window, Separated, Divorced, Never Married.

Socioeconomic status (SES). During all four waves, information was collected on personal annual income and highest education level completed. Income was collected in increments of $\$ 4,999$, starting from $0-4,999$ to 50,000 or more, on a scale from 1 to 11 . For the data analysis, income was merged into six ordinal variables $(1=0-9,999,2=10,000-29,999,3=30,000-39,999,4=40,000-$ 49,999, $5=50,000$ and over, $6=$ Don't remember). Data on education was collected using 9 ordinal levels ( $1=$ Less than high school, $2=$ High school diploma, 3= High school equivalency (GED), 4= High school for adults, 5= Some training/college after high school, 6= Some training without finishing HS, 7= 
Bachelor's (4 yr. college) degree, 8= Graduate/Professional studies, 9= Other). For the data analysis, education was merged into 4 ordinals: 1= Less than high school, 2= High school/GED, 3= Bachelor, and 4= Graduate/Professional.

Nativity. Participants were asked if they were born in the US or outside the US (yes/no). If born outside of the US, they were then asked to provide their country of birth. The variable was dichotomized into $1=Y e s$ and $0=$ No.

\section{Alcohol misuse.}

Alcohol misuse was scored using items from the Health and Daily Living Form (Billings, Cronkite, \& Moos, 1983) in order to assess each participant's alcohol use frequency during the 12 months prior to assessment and, in part, to determine dyad membership. The measure includes questions for beer, wine, and hard liquor ("How often did you drink beer during the last 12 months?") using an 8-point Likert-type scale from 1 (Never uses) to 8 (Uses every day). Alcohol misuse was defined as having at least one of the following: (a) 4-5 glasses or more of wine on any one occasion several times a month; (b) 3-4 cans/bottles or more of beer on any one occasion several times a month; (c) 3-4 drinks or more on any one occasion with $4 \mathrm{oz}$. of alcohol per drink several times a month; and/or (d) 5-7 drinks or more in one occasion with 2 oz. of alcohol per drink several times a month. For the analysis, alcohol misuse was recoded into a binary variable of $1=$ User and $2=$ Non-User . 


\section{Data Analytic Plan}

\section{Preliminary analysis.}

Statistical analyses were completed using SPSS 24 (Field, 2013).

Descriptive statistics were performed for all variables to summarize data and profile all variables. Variables measured on non-metric scales (categorical variables) were summarized in terms of proportions while those measured in metric scales (continuous variables) will be summarized in terms of means and standard deviations. Examination of the distribution forms were pursued through graphical methods and summary statistics of higher order moments (skewness and kurtosis).

Frequency distributions for all continuous variables were conducted to determine if they violated the assumption of normality. According to Kline (2005), continuous variables were deemed non-normal if they yielded absolute skewness and kurtosis values greater than 3.0 and 8.0 , respectively.

Statistical tests for were conducted to assess the potential influence of covariates in the research model. Dependent covariates were chosen in accordance to the literature and included age, income, education, health insurance, marital status, acculturation and alcohol misuse. Spearman's rho correlation analyses were used to examine the influence of continuous covariates age, and acculturation on study outcomes (Mothers HIV sexual risk behaviors, Mothers HIV testing, and Mothers HIV Counseling). An analysis of variance (ANOVA) examined the potential influence of the categorical variables education and income. A Chi Square test identified associations between binary covariates 
health insurance, marital status, and alcohol misuse on older Latina mother HIV risk outcomes.

\section{Primary analyses.}

The study utilized a recurrent cross-sectional research design (Grossoehme, \& Lipstein, 2016). Recurrent cross-sectional analysis (ReC-SA) can be thought of as a series of smaller studies given that at each time point data from all participants are analyzed as an independent unit. After this analysis is completed, a second analysis focuses on differences and similarities between time points. ReC-SA describes differences when comparing time points. The level of analysis may be done across the entire sample or a subsample, and may be done upon completion of each time point. ReC-SA is appropriate when questioning how beliefs, such as health behaviors, change over time (Grossoehme, \& Lipstein, 2016). A recurrent cross-sectional approach is best utilized for questions that focus on comparing discrete time points or where logistical challenges prevent examining changes overtime. In the present study, recurrent cross-sectional analyses was utilized in place of more traditional longitudinal techniques due to sample size limitations that did not provide enough power to examine changes in trajectory overtime. Such analysis would have required approximately a sample of $N=800$ ( $n=400$ dyads) with a medium (0.20) effect size and 80\% power (Hedeker, Gibbons, \& Waternaux, 1999). Therefore Recurrent cross-sectional analyses was conducted across four time points $\left(T_{0}, T_{1}\right.$, $\mathrm{T}_{2}$, and $\left.\mathrm{T}_{3}\right)$. 
The overarching aims of the study were to (a) examine associations between HIV risk behaviors of adult Latina daughters and those of their older Latina mothers and (b) test the moderation effect of mother-daughter attachment on those associations.

Recurrent cross-sectional moderation models across $T_{0}, T_{1}, T_{2}$, and $T_{3}$ were conducted using PROCESS v2.16, a path analysis modeling macro tool available for SPSS (Hayes, 2012). PROCESS "is widely used through the social, business, and health sciences for estimating direct and indirect effects in... two and three way interactions in moderation models" (Hayes, n.d., para. 1); PROCESS simplifies the moderation analysis (Hayes, n.d.). Figure 4 shows the PROCESS moderation model where the effect of some predictor $(\mathrm{X})$ on some outcome $(\mathrm{Y})$ is influenced, or dependent, on a moderator (M) (Hayes, 2012). For the current study PROCESS estimates the direct effects of predictors (adult Latina daughter HIV sexual risk behaviors, HIV testing, and HIV counseling) on outcomes (older Latina mother HIV sexual risk behaviors, HIV testing, and HIV counseling) while simultaneously examining the moderating influence of mother-daughter attachment on those associations. The direct and indirect effects of adult Latina daughters having received HIV counseling across all three outcomes was examined. There is evidence that adult daughters seek out health related information and share with their mothers (Browne \& Chan, 2012; Kratzke, Amatya, \& Vilchis, 2014; Mosavel, 2009; Mosavel, \& Genderson, 2016; Mosavel, \& Thomas, 2009). Therefore, effects of predictor (adult Latina daughter HIV counseling) were also examined on outcomes (older Latina mother HIV testing 
and HIV sexual risk behaviors). Hair states that a moderator effects third variables or constructs that change the relationship between two variables or constructs that are related. Meaning, a moderator that shows the association between variables, changes depending on the amount of another variable that was added to the model (Hair, Black, Babin, Anderson, \& Tatham, 2006).

When traditionally examining interaction effects, first order predictors and moderator terms are mean centered (Little, Card, Bovaird, Preacher, \& Crandall, 2007). It is recommended that continuous variables be centered when conducting moderation analyses because the predictor and moderator variables are highly correlated with the product terms created for them (Little et al., 2007). This can create instability in the values of the estimated regression weights (Aiken \& West, 1991; Cohen, Cohen, West, \& Aiken, 2003; Jaccard, Turrisi, \& Wan, 1990). Several studies have shown that if the first order variables are mean centered, meaning transformed from raw score to a deviation score scaling by subtracting the variable mean from all observations, the result product term is minimally correlated/uncorrelated with the first order variables, if the variables are reasonably bivariate normal (Little et al., 2007). Next, product terms are usually created between the predictor and moderator terms representing their interaction.

However, PROCESS version 2.16 automatically mean centers predictor and moderator terms and creates interaction terms. In addition, PROCESS recognizes binary outcomes without having to incorporate additional syntax. It should be noted that PROCESS v2.16 only produces confidence intervals for 
unstandardized regression coefficients; therefore, results are presented in unstandardized values $(B)$.

\section{Human Participants Consideration}

This Human Subjects Research falls under Exemption (4): "Research involving the collection or study of existing data, documents, records, pathological specimens, or diagnostic specimens, if these sources are publicly available or if the information is recorded by the investigator in such a manner that subjects cannot be identified, directly or through identifier linked to the subjects"

As a secondary data analysis, the data will have already been collected under the auspices of Institutional Review Board (IRB) approved procedures at Florida International University (FIU), thus, human subjects' issues have been addressed. This Methods for this study were all approved by the IRB at FIU. As secondary data analysis, this study posed no risk of harm to participants.

Nevertheless, the dataset was stripped of all identifiers.

All participants signed an informed consent form which was read outload in either English or Spanish, depending on the participant's preference. Consent specified participant's rights to confidentiality, their right to terminate participation at any time, and contact information for the principle investigator in case participants had additional comments or complaints. All interviewers completed sensitivity and confidentiality training. Strict measures were taken to ensure the database was secure. Contact information sheets were maintained locked without access to anyone not assigned to the study and completed data was 
maintained in double coded electronic storage accessible only to the principle investigator. 


\section{CHAPTER IV. RESULTS}

This study examined the (a) influence of daughter's HIV risk behaviors may have on that of their mother's HIV risk behaviors and $(b)$ whether attachment plays a moderating role on those associations influence. The data analytic plan for the present study consisted of: 1) obtaining descriptive statistics of the sample, 2) checks for non-normality, 3) testing for the potential influence of covariates in the model, 4) a series of recurrent cross-sectional path analysis testing the direct effects of adult Latina daughters HIV risk on their older Latina mother's HIV risk, and the moderating influence of mother-daughter attachment on that association.

\section{Preliminary Analysis}

A variety of analytical techniques and procedures were conducted in order to determine possible data outliers, missing data, or non-normality. Below are the results of the preliminary analysis.

\section{Descriptive statistics.}

Tables 3 thru 12 presents the frequency and distributions for all key observed study variables. Results indicated all continuous variables were normally distributed. Sample demographics revealed $72 \%$ of participants $(N=158)$ were foreign born ( $86 \%$ of mothers [ $n=79]$ and $58 \%$ of daughters [ $n=79])$ had emigrated to the United States (See Table 3). In regard to country of origin, $30.4 \%$ of the participants were Cuban, $10 \%$ from Colombian, $11 \%$ from Peruvian, and over $8 \%$ from Puerto Rican (See Table 2). Overall, participants reported low levels of education with $54 \%$ having less than high school education, $28 \%$ had a 
GED or high school diploma, $18 \%$ indicated education levels beyond high school education. Table 3 displays the samples education levels aggregated by mothers and daughters. At baseline, participants reported relatively low income levels with over half the sample (51.3\%) having personal incomes of less than $\$ 10,000$ per year and $90 \%$ of participants reporting annual incomes of less than $\$ 30,000$ per

year (See Table 3). Lastly, the mean participant age was 58.5 years $(S D=7.48$, range of 50 to 88 years) for mothers, and 31.3 years $(S D=9.73$, range of 18 to 55 years) for daughters (See Table 4).

At baseline $38 \%$ of mothers reported at least one sexual behavior in the last 12 months (Table 5), but almost half (49.4\%) reported never having been tested for HIV (Table 6). Additionally, Table 7 shows $65.8 \%$ of mothers said no health care provider had spoken to them about HIV prevention or safe sex (counseling). Tables $8-10$ detail daughters' behaviors. Over $82 \%$ of adult daughters reported some form of risky sexual behavior during the past 12 months, with $80 \%$ reporting having been tested for HIV. As for HIV counseling, Table 12 shows $62 \%$ of daughters said they had spoken to a health care professional about HIV prevention or safe sex.

\section{Missing data.}

There were minimal amounts of missing data. Of the 474 dependent and independent predictor variables, only one was missing. As such, listwise deletion was used to deal with missing data. 


\section{Analysis of Sociodemographics as covariates.}

Statistical tests were conducted to assess the potential influence of covariates in the research models. Variables were evaluated for their influence on dichotomous study outcomes (older Latina mother's HIV risk, HIV testing, and HIV counseling) across each time point $\left(T_{0}, T_{1}, T_{2}\right.$, and $\left.T_{3}\right)$.

An analysis of variance (ANOVA) was used to evaluate the potential influence of categorical variables education and income. Chi-square analyses were used to analyze binary covariates (health insurance, marital status, and alcohol misuse). Spearman rho correlations assessed continuous variables age and acculturation. Results indicated younger age was associated with greater involvement in HIV sexual risk behaviors at baseline and $\mathrm{T}_{1}\left(\mathrm{~T}_{0}[r=-.35, p=.002]\right.$; $\left.\mathrm{T}_{1}[r=-.38, p=.001]\right)$. Married women were more likely to engage in HIV sexual risk behaviors across all time points $\left(\mathrm{T}_{0}: \Delta \mathrm{X}^{2}[1]=9.78, p=.002 ; \mathrm{T}_{1}: \Delta \mathrm{X}^{2}[1]=\right.$ 21.64, $\left.p=<.001 ; \mathrm{T}_{2}: \Delta \mathrm{X}^{2}[1]=31.94, p=<.001 ; \mathrm{T}_{3}: \Delta \mathrm{X}^{2}[1]=20.23, p<.001\right)$. At baseline married women were less likely to have been tested for HIV ( $T_{0}: \Delta \mathrm{X}^{2}$ [1] $=5.31, p=.021)$ Table 3 shows number of married mothers and daughters. No other potential covariates were significantly associated with the study outcomes. Subsequent primary analysis included marital status and age as covariates.

\section{Primary Analyses}

A series models with 10,000 bootstrap iterations were tested with the SPSS macro PROCESS v2.16. Figures 5-8 presents results for the series of recurrent cross sectional moderation models tested at each time point. Results 
are presented in unstandardized values $(B)$. No significant direct and moderating effects were found in the proposed research models.

A series of post-hoc analyses were subsequently conducted. First, the total scale score for the attachment variable was disaggregated by subscale (Trust, Communication, and Alienation Subscale). Interaction effect for each attachment subscales were tested independently in the research model. No significant interaction effects were found for any of the subscales.

Next, post hoc analyses tested the proposed research model on the full parent study sample ( $N=130$ mother-daughter dyads) of the last time point. The last time point $\left(T_{3}\right)$ was utilized for this analyses to increase the probability for mothers in the dyads being 50 years of age or older due to time laps of approximately 10 years and determine if the increases sample size would impact study findings. Cross sectional analyses on the $N=130$ dyads followed the same procedures as those previously indicated for the original $N=79$ dyads with regard to preliminary data analyses. Descriptive statistics indicated that ages for the mothers in the post hoc analyses ranged from 41-97 ( $M=60.02, S D=9.97)$. Seventeen dyads included mothers under the age of 50 . These cases were included in the exploratory analyses to maximize sample size.

Post-hoc analyses indicated that Latina mothers who were married $(B=$ 2.95, $p<.001)$ engaged in greater HIV sexual risk behavior, also higher income $(B=.18, p=.03)$ was associated with a greater likelihood of having received HIV counseling. No other significant covariates were evident in the post-hoc analyses. Interaction effects for the full attachment scale, as well as the individual 
subscales (Trust, Communication, and Alienation Subscales), were examined. No direct effects of predictors (adult Latina daughter HIV sexual risk behaviors, HIV testing, and HIV counseling) on outcomes (older Latina mother HIV sexual risk behaviors, HIV testing, and HIV counseling) were found. Also, no significant moderating effects for mother-daughter attachment were found on those associations. 


\section{CHAPTER V. DISCUSSION}

\section{Summary of Results}

The current study examined the associations between adult Latina daughter's HIV risk behaviors and that of their older mothers during four time points and whether such behaviors were moderated by the mother-daughter attachment. The purpose was to take a step toward exploring the possibility of a new family prevention method for older Latina women who may be at risk for HIV infection. Despite Latinx cultural beliefs like marianismo, that place pressure on Latinas to be remain loyal, it also pressures them to be submissive to their partners, disregard their infidelities, and not attempt to negotiate condom use; beliefs like machismo encourage Latinos' infidelities; placing both at risk for HIV infection, while other cultural beliefs silence and stigmatize the family, biological factors put the older women at higher risk. This combination of cultural and biological factors place older Latinas at risk to HIV infection and creating a public health problem. Therefore, to address the current gap in the literature, the current study used secondary data from a longitudinal Latina mother-daughter study to test two aims. Aim 1 of the study was to examine the association between recurrent HIV risk behaviors of adult Latina daughters and also that of their older Latina mothers (50 and over year of age). It was hypothesized that recurrent HIV risk behaviors among adult Latina daughters would be positively associated with the recurrent HIV risk behaviors of their older Latina mothers. Results of the analysis found no support for this hypothesis. Aim 2 examined the interaction effect that the mother-daughter attachment moderator had on associations 
between the recurrent HIV risk behaviors of adult Latina daughters and those of their older Latina mothers. The first hypothesis of Aim 2 was that older mothers with higher attachment levels to their adult daughters would show a stronger association between recurrent HIV risk behaviors of adult Latina daughters and those of their older Latina mothers. Results of the analysis found no support for this hypothesis. The second hypothesis of Aim 2 was that mothers with lower attachment levels to their daughters would show weaker association between recurrent HIV risk behaviors of adult Latina daughters and those of their older Latina mothers. Results of the analysis found no support for this hypothesis. However, consistent with the literature age was found to be significantly negatively correlated with HIV sexual risk behaviors (CDC, 2017c; Craig, 2007), HIV testing and HIV counseling (Craig, 2007) (See Table 4). Additionally, marital status was also significantly correlated with HIV sexual risk behaviors. Married participants were found to have higher HIV sexual risk behaviors, this finding was driven by the variable measuring unprotected sex.

\section{HIV sexual risk behaviors.}

Whether direct or indirect, communication about sex among Latina mothers and daughters seems to vary. In some studies, pregnant Latina teenagers report having had less sexual communication with their parents than reports from nonpregnant teens (Adolph, Ramos, Linton, \& Grimes, 1995; Baumeister, Flores, \& Marin, 1995). However, a 1994 study showed that Latina adolescents report higher levels of communication about sex with their mothers than did adolescent Latinos (Hovell, Sipan, Blumberg, Atkins, Hofsteter, \& 
Kreitner, 1994). Yet more recent studies found that although Latina daughters report having better communication with their mothers than Latino sons, findings suggest the communication is less about sex and more about values, beliefs, and relationships (Mena et al., 2008; Raffaelli \& Green, 2003; Romo et al., 2002). Thus, in support of the study aims, the current study examined the association between adult Latina daughter risky sexual behaviors and that of their older mothers'. Data analysis did not support the hypothesis that adult Latina daughters' sexual risk behaviors would be associated with that of their mothers, suggesting possible daughter-initiated behaviors or influence. Lack of significance in the analysis may imply that lack of communication about risky sexual behaviors among Latina mothers and their adolescent daughters may continue bi-directionally into adulthood.

Although the hypothesis for aim 1 was not supported, results of the analysis were consistent with the literature and showed younger age as a predictor to HIV sexual risk behaviors. Additionally, being married was also found to be a predictor to HIV sexual risk behavior. It is likely sex without a condom is driving the significance with married status. However, as stated in Chapter II, Latinx machismo and marianismo traditional gender norms place pressure on Latinas to disregard their partners' infidelities and not negotiate condom use; thus, increasing these women's risk of HIV infection via heterosexual sex with their primary partners. 


\section{HIV testing.}

Adult daughters, including adult Latina daughters, influence their mothers' health screening behaviors by way of sharing of information with their mothers, encouraging them to be tested and proactively seek out information on healthrelated preventions (Browne \& Chan, 2012; Kratzke, Amatya, \& Vilchis, 2014; Mosavel, 2009; Mosavel, \& Genderson, 2016; Mosavel, \& Thomas, 2009; Washington et al., 2009). However, studies have been limited to cancer screenings. Although older Latinas' HIV testing is associated to HIV counseling from health care providers (Craig, 2007), until the current study, to date, no studies have looked at possible associations between older Latina's HIV testing and their adult daughters' testing behaviors. No correlation was found between adult daughter's HIV testing and that of their older mother's. Therefore, analysis for the current study did not support the hypothesis that adult Latina daughters' HIV testing would be associated with that of their older mother's. However, consistent with the literature (Craig, 2007), results showed age as a significant predictor to HIV testing.

\section{HIV counseling.}

Although HIV counseling from health care providers is a predictor of HIV testing (Craig, 2007), language gaps may make HIV counseling futile. Language gap problems may be more problematic in less diverse geographical areas of the country where it is more difficult to find bilingual physicians or translators. However, even in Miami-Dade County, Florida, where $64 \%$ of the population speaks Spanish at home (Statistical Atlas, 2015), differences in health care due 
to primary language are evident. In fact, a 2005 survey found that Miami residents who reported speaking English at home were more likely to have a regular dentist (Graham, Tomar, \& Logan, 2005). Nevertheless, ensuring reduction of ethnic/racial health disparity depends on both language as well as cultural competence (Huey, Tilley, Jones, \& Smith, 2014). A study with 546 adult daughters (50\% Latinas) Kratzke and colleagues found that daughters passively and actively seek out health-related information, like from the internet or formal education and share it with their mothers (2014). Daughters not only bridge language gap, but also dismiss health myths for their mothers (Kratzke, Amatya, \& Vilchis, 2014). Such studies show that daughters are serving as brokers of language, information, and culture to their mothers.

In support of aim 1, the current study appropriately examined the association between adult Latina daughters' HIV counseling and their older mother's HIV counseling as well as the mother's HIV testing and risky sexual behaviors. However, data analysis for the current study did not support the hypothesis that adult Latina daughters' HIV counseling was associated with their older mothers' HIV counseling, HIV testing, or sexual risk behaviors.

Because Latinx parents and children hold such taboos about discussing sex (Gil \& Vazquez, 1996), it is likely that messages gathered by adult Latina daughters that warn about risky sexual behaviors or promote HIV prevention are not being passed down to their mothers due to cultural taboos that have existed throughout adolescents (Mena et al., 2008). Analysis of the current study data showed no association between adult Latina daughters HIV counseling and that 
of their mother's counseling, testing, or risky sexual behaviors. Consistent with the literature (Craig, 2007), HIV counseling was negatively correlated with age. As with risky sexual behaviors, results may suggest that communication about taboo topics continue to be taboo for Latina mothers and daughters even into adulthood.

\section{Future Recommendations}

Research.

The literature has a wealth of studies regarding mother-daughter studies. Such studies include examining risky and protective sexual behaviors, and substance use. Many of them also include attachment and cultural beliefs as factors to risk behaviors. Moreover, several of the same studies also examine dyadic samples. However, to date, a small amount has focused on Latinas and an even smaller amount on adult Latina dyads. Given the rising Latinx population, older adults, and HIV risk behaviors among older adults, it is critical that more research is focused on this population as they age and require multiple services. Further research is needed to further understand the sexual risks of

older less-acculturated Latina women. Additionally, the lack of significance in the current study suggests that the sex taboos that exist in Latino cultures are being carried over into adulthood by daughters and their older mothers. Researchers need to consider cultural taboos when working with older Latinas and insure candid responses. Self-administered interviews that are more likely to promote honest responses from this population should be considered. 


\section{Preventions.}

Future prevention efforts with older Latinas should include development of a family-based, age, and culturally-appropriate prevention strategies that include adult daughters as broker to information and cultural barriers. Considering previous studies that examine adult daughters influence over their mothers' health-related behaviors (Browne \& Chan, 2012; Kratzke, Amatya, \& Vilchis, 2014; Mosavel, 2009; Mosavel, \& Genderson, 2016), daughters do seem to be able to influence their mothers' health-related decisions. In fact, Mosavel and Thomas (2009) found that $100 \%$ of Latina daughters responded they would absolutely give their mothers health advice, compared to $63 \%$ African American daughters. The main reason for the difference was that African American daughters felt it was something their mothers already knew or should have known (Mosavel \& Thomas, 2009); while Latina daughters suggested their mothers may not have known. Future prevention strategies must keep in mind barriers that keep older Latinas from obtaining reliable information about HIV risk such as language gaps and cultural factors that keep Latinas from speaking openly about sex-related issues. Often older parents are less acculturated than children that may have been raised in the U.S. Prevention programs should include adult daughters as brokers for their mothers. Strategies may include communication techniques that facilitate adult daughters to engage their older mothers about risky sexual behaviors. To date, no such prevention strategies are in effect. 


\section{Policy.}

As an HIV prevention strategy, in 2006 , the CDC recommended routine testing for all patients 13 to 64 years of age in health care settings (CDC, 2006). This was an effort to increase testing as well as break down barriers that prevent individuals from testing by opening the lines of communication for HIV testing. However, this policy excludes sexually active adults over age 64 which creates the assumption that adults over 64 are not sexually active and thus not at risk for HIV infection. Yet older adults remain active well into their 80's and moreover engage in risky sexual behaviors (Johnson, 2013; Falvo \& Morman, 2004; Schick et al., 2010) and find it difficult to speak openly to share with their physicians (Lindau, Leitsch, Lundberg, \& Jerome, 2006). The Infectious Diseases Society of America has taken recommendations further by recommending routine HIV testing for all sexually active adults (Lubinski et al., 2009).

In 2010, Pinkerton and colleagues conducted a study on the cost of rapid HIV testing in hospitals. The study found the average per-test cost of rapid HIV testing and counseling was $\$ 48.07$ for a HIV-negative test and $\$ 64.17$ for a preliminary-positive test. Counseling accounted for $38.4 \%$ of the total cost (Pinkerton, Bogart, Howerton, Snyder, Becker, \& Asch, 2010). Lowering home HIV test costs may help to boost the rate of HIV testing among low income populations where HIV resides most. Lower costs may also encourage healthcare setting to promote testing to patients more often. Additionally, affordable home tests can encourage individuals to test more often in the privacy of their own homes. Economical testing techniques and procedures are especially 
important to individuals at high risk for HIV infection. However, despite the cost of counseling, it is important that patients are aware of risk and protective methods and know how to gain access to HIV treatment services when necessary.

\section{Implications for Social Work}

Social workers are organizers. Through organization, they can generate changes to local or federal policies as well as develop prevention programs that empower individuals and families. At a macro level, social workers can advocate for policies that provide health equality to individuals and communities, such as expanding HIV testing to all sexually active individuals. Moreover, social workers are also researchers contributing to public health, including HIV research. Social work HIV research contribute to intervention strategies, program implementations, and evaluations that are use at both micro and macro levels.

The National Association of Social Workers (NASW) and the Substance Abuse and Mental Health Services Administration (SAMHSA) joined together to contribute to the goals of the National HIVIAIDS Strategy. The strategies are to 1) reduce the number of new HIV infections, 2) increase access to care and enhance health outcomes, and 3) reduce HIV-related health disparities (NASW, 2014). Because testing provides awareness of status, and awareness of seropositive status decreases risky sexual behaviors, subsequently testing reduces the number of new HIV infection. As social workers, it is important to assess to what degree such services are being utilized and to reach out to the hardest to reach communities that have been historically underserved to ensure 
health equity. Social workers are in a unique position to greatly help reduce the number of new HIV infections in both macro and micro levels.

As professionals trained to work with individuals, families, and communities with a variety of cultures, social workers play roles in helping to empower those who may have been disenfranchised, such as women, immigrants, children, and elders. Social workers who work with families can help bridge the cultural gap between family members, such as Latina mothers and daughters. Encouraging open lines of communications about risky and protective sex behaviors between parents and children, results in children being more open with their parents about sex. Such strategies may lead to open communications about sex into adulthood. Additionally, medical social workers are in a position where they can partner with health care professionals to reduce stereotypes held about sex and older adults. Such efforts can lead to increases in HIV testing, counseling and a reduction of stigma. Finally, social workers must continue HIV research efforts that contribute to breaking down barriers and address sociocultural challenges that place older Latinxs at risk for HIV infection.

This study adds to the literature and scientific field of social work and public health. Social workers and public health researchers examine and explore HIV interventions, preventions strategies, and evaluate programs in place being implemented for current or future use. This study contributes to such research by exploring the role of family members as deterrents to risky sexual behaviors and adult daughter-initiated upward communication as a technique to promote healthy behaviors among older adults. 


\section{Limitations}

Several limitations of the study should be considered. The most significant limitation to the current study was sample size. Although the parent study maintained a $96 \%$ retention rate, once inclusion criteria for the current study were met, the data yield a small sample size. Future research would benefit greatly from a larger more representative sample that could withstand more elaborate analysis and provide confidence in the findings. Although data for the present study was collected using face to face interviews, given cultural taboos addressed in Chapter II, self-administered questionnaires (SAQ) would reduce social desirability and possible interviewer bias.

In addition to sample size and interview methods, measurement instruments may have also posed limitations. The study attempted to test moderation of the interaction of the mother-daughter relationship using the Inventory of Parent and Peer Attachment (IPPA) scale (Armsden \& Greenberg, 1987). Although IPPA is widely used among parents and under age children, further development of instruments to assess adult parent-child attachment may be warranted. Furthermore, although interviewers were extensively trained in building rapport as well as maintaining confidential information as required by the Institutional Review Board, sexual behaviors could not be confirmed. Instruments relied heavily on subject self-reporting. Accuracy of answers about sexual activities from a sample of Latina women may be difficult to ascertain given cultural taboos. Some of the data may have been strengthened with the availability of information from other sources, such as HIV test records. Future 
studies on risky sexual behaviors of older Latinas may benefit from being able to gain information from other reliable sources, provide self-administered interviews, and a random and larger sample size.

\section{Conclusion}

This study aimed to advance the area of social welfare by exploring innovative strategies that would help reduce risky sexual behaviors of older Latinas. Advancing strategies to reduce the risky sexual behaviors of older Latinas could lead to reductions of HIV infections among a hidden population. Embedded cultural factors and cultural barriers make older Latinas a challenging population for prevention strategies. However, this study intended to show that by capitalizing on the same cultural values that create barriers to HIV preventions, such as familismo, older Latina women could be reached through their adult daughters. Most studies examining protective familial influences on Latina substance use and/or HIV risk behaviors, focused on adolescent samples (Robles et al., 2006; Romo et al., 2002). Examining the associations between older Latina mothers' and their adult daughters' risky sexual behaviors would build on existing literature that examine either mothers and adolescent daughter dyads or the health-related behaviors of adult mother and daughters.

Although the current study did not yield results as hypothesized, the findings underscored the impact, strength, and trajectories of the sexual taboos that exist in Latinx families and carry over into adulthood. Moreover, such results should not be discouraging, but instead inspire further research that measures and examines Latinx family communication strategies and factors that empower 
Latinas to break through the cultural taboos that place them at risk for HIV infection. 
Tables and Figures 
Table 1.

Inclusion Criteria for Current Study

\begin{tabular}{ll}
\hline Criteria & \multicolumn{1}{c}{ Inclusion } \\
\hline Age & 18 and over (Daughters); 50 and over (Mothers) \\
Country of Birth & U.S. Latina America, or Caribbean \\
Interview & Consent to $2 / 3$ hr. interview \\
Participation & Participant of Parent Study \\
Contact Information & Willing to provide 2 contacts
\end{tabular}

Table 2.

Participants Identifying Country

\begin{tabular}{lcccc}
\hline & $n$ & $\%$ & Valid \% & Cumulative \% \\
\hline Mexico/Mex-Am/Chicana & 8 & 5.1 & 5.1 & 5.1 \\
Puerto Rico & 13 & 8.2 & 8.2 & 13.3 \\
Cuba & 48 & 30.4 & 30.4 & 43.7 \\
Nicaragua & 4 & 2.5 & 2.5 & 46.2 \\
Honduras & 4 & 2.5 & 2.5 & 48.7 \\
Venezuela & 4 & 2.5 & 2.5 & 51.3 \\
Colombia & 16 & 10.1 & 10.1 & 61.4 \\
Ecuador & 4 & 2.5 & 2.5 & 63.9 \\
Peru & 17 & 10.8 & 10.8 & 74.7 \\
Bolivia & 2 & 1.3 & 1.3 & 75.9 \\
Argentina & 5 & 3.2 & 3.2 & 79.1 \\
Other & 33 & 20.9 & 20.9 & 100.0 \\
Total & 158 & 100.0 & 100.0 & \\
\hline
\end{tabular}


Table 3.

Descriptive Statistic for Mothers and Daughters

\begin{tabular}{|c|c|c|c|c|c|c|c|c|}
\hline & \multicolumn{3}{|c|}{ Mothers } & \multirow[b]{2}{*}{ Cumulative $\%$} & \multirow[b]{2}{*}{$\mathrm{n}$} & \multicolumn{2}{|c|}{ Daughters } & \multirow[b]{2}{*}{ Cumulative $\%$} \\
\hline & $n$ & $\%$ & Valid \% & & & $\%$ & Valid \% & \\
\hline \multicolumn{9}{|l|}{ Nativity } \\
\hline Foreign born & 68 & 86.1 & 86.1 & 86.1 & 46 & 58.2 & 58.2 & 58.2 \\
\hline US Born & 11 & 13.9 & 13.9 & 100.0 & 33 & 41.8 & 41.8 & 100.0 \\
\hline Total & 79 & 100.0 & 100.0 & & 79 & 100.0 & 100.0 & \\
\hline \multicolumn{9}{|l|}{ Education } \\
\hline Less than HS & 41 & 51.9 & 51.9 & 51.9 & 44 & 55.7 & 55.7 & 55.7 \\
\hline High School & 20 & 25.3 & 25.3 & 77.2 & 24 & 30.4 & 30.4 & 86.1 \\
\hline Bachelor & 13 & 16.5 & 16.5 & 93.7 & 8 & 10.1 & 10.1 & 96.2 \\
\hline Grad/Professional & 5 & 6.3 & 6.3 & 100.0 & 3 & 3.8 & 3.8 & 100.0 \\
\hline Total & 79 & 100.0 & 100.0 & & 79 & 100.0 & 100.0 & \\
\hline \multicolumn{9}{|l|}{ Marital Status } \\
\hline No partner & 62 & 78.5 & 78.5 & 78.5 & 60 & 75.9 & 75.9 & \\
\hline Married/Partner & 17 & 21.5 & 21.5 & 100.0 & 19 & 24.1 & 100.0 & \\
\hline Total & 79 & 100.0 & 100.0 & & 79 & 100.0 & & \\
\hline \multicolumn{9}{|l|}{ Income at Baseline } \\
\hline $0-4,999$ & 19 & 24.1 & 24.1 & 24.1 & 29 & 36.7 & 36.7 & 36.7 \\
\hline $5,000-9,999$ & 19 & 24.1 & 24.1 & 48.1 & 14 & 17.7 & 17.7 & 54.4 \\
\hline $10,000-14,999$ & 14 & 17.7 & 17.7 & 65.8 & 9 & 11.4 & 11.4 & 65.8 \\
\hline $15,000-19,999$ & 3 & 3.8 & 3.8 & 69.6 & 11 & 13.9 & 13.9 & 79.7 \\
\hline $20,000-24,999$ & 3 & 3.8 & 3.8 & 73.4 & 9 & 11.4 & 11.4 & 91.1 \\
\hline $25,000-29,999$ & 9 & 11.4 & 11.4 & 84.8 & 2 & 2.5 & 2.5 & 93.7 \\
\hline $30,000-34,999$ & 3 & 3.8 & 3.8 & 88.6 & 1 & 1.3 & 1.3 & 94.9 \\
\hline $35,000-39,999$ & 2 & 2.5 & 2.5 & 91.1 & 2 & 2.5 & 2.5 & 97.5 \\
\hline $40,000-44,999$ & 2 & 2.5 & 2.5 & 93.7 & 1 & 1.3 & 1.3 & 98.7 \\
\hline $45,000-49,999$ & 2 & 2.5 & 2.5 & 96.2 & 0 & $\mathrm{n} / \mathrm{a}$ & $\mathrm{n} / \mathrm{a}$ & $\mathrm{n} / \mathrm{a}$ \\
\hline 50,000 or more & 2 & 2.5 & 2.5 & 98.7 & 1 & 1.3 & 1.3 & 100.0 \\
\hline Does not remember & 1 & 1.3 & 1.3 & 100.0 & 0 & 100.0 & 100.0 & \\
\hline Total & 79 & 100.0 & 100.0 & & & & & \\
\hline
\end{tabular}

Table 4.

Age at Baseline, $N=158$

\begin{tabular}{lcc}
\hline & \multicolumn{2}{c}{ Participants } \\
\cline { 2 - 3 } & Mothers & Daughters \\
\hline$n$ & 79 & 79 \\
Mean & 58.51 & 31.32 \\
Median & 56.00 & 29.00 \\
Mode & 56 & $25^{\mathrm{a}}$ \\
Std. Deviation & 7.486 & 9.729 \\
Range & 38 & 37 \\
Minimum & 50 & 18 \\
Maximum & 88 & 55 \\
\hline
\end{tabular}

a. Multiple modes exist. The smallest value is shown 
Table 5.

Mother-HIV Sexual Risk Behaviors* (Did the participant have any HIV sexual risk behaviors in the last 12 months?)

\begin{tabular}{llrrc}
\hline & & & & Cumulative \\
& $n$ & \multicolumn{1}{c}{$\%$} & Valid \% & Percent \\
\hline No & 49 & 62.0 & 62.0 & 62.0 \\
Yes & 30 & 38.0 & 38.0 & 100.0 \\
Total & 79 & 100.0 & 100.0 & \\
\hline
\end{tabular}

*HIV Sexual Risk Behaviors include: sex without condoms, sex under the influence, multiple sex partners, or all

Table 6.

Mother-HIV Testing (Have you ever been tested for HIV?)

\begin{tabular}{lllll} 
& & & & Cumulative \\
& $\mathrm{n}$ & $\%$ & Valid \% & Percent \\
\hline No & 39 & 49.4 & 49.4 & 49.4 \\
Yes & 40 & 50.6 & 50.6 & 100.0 \\
Total & 79 & 100.0 & 100.0 & \\
\hline
\end{tabular}

Table 7.

Mother-HIV Counseling (Has your doctor or any health professional spoken to you about HIV prevention or safe sex, in the last 12 months?)

\begin{tabular}{lcccc}
\hline & & & & Cumulative \\
& $n$ & $\%$ & Valid \% & Percent \\
\hline No & 52 & 65.8 & 66.7 & 66.7 \\
Yes & 26 & 32.9 & 33.3 & 100.0 \\
Total & 78 & 98.7 & 100.0 & \\
\hline
\end{tabular}

Table 8. 
Daughter-HIV Sexual Risk Behaviors* (Did the participant have any HIV sexual risk behaviors in the last 12 months?)

\begin{tabular}{lcrcc}
\hline & & & Valid & Cumulative \\
& $\mathrm{n}$ & \multicolumn{1}{c}{$\%$} & \multicolumn{1}{c}{$\%$} & Percent \\
\hline No & 14 & 17.7 & 17.7 & 17.7 \\
Yes & 65 & 82.3 & 82.3 & 100.0 \\
Total & 79 & 100.0 & 100.0 & \\
\hline
\end{tabular}

*HIV Sexual Risk Behaviors include: sex without condoms, sex under the influence, multiple sex partners, or all

Table 9.

Daughter-HIV Testing (Have you ever been tested for HIV?)

\begin{tabular}{lcccc}
\hline & & & & Cumulative \\
& $\mathrm{n}$ & $\%$ & Valid \% & Percent \\
\hline No & 16 & 20.3 & 20.3 & 20.3 \\
Yes & 63 & 79.7 & 79.7 & 100.0 \\
Total & 79 & 100.0 & 100.0 & \\
\hline
\end{tabular}

Table 10.

Daughter-HIV Counseling (Has your doctor or any health professional spoken to you about HIV prevention or safe sex, in the last 12 months?)

\begin{tabular}{lllll}
\hline & & & & Cumulative \\
& $\mathrm{n}$ & $\%$ & Valid \% & Percent \\
\hline No & 30 & 38.0 & 38.0 & 38.0 \\
Yes & 49 & 62.0 & 62.0 & 100.0 \\
Total & 79 & 100.0 & 100.0 & \\
\hline
\end{tabular}

Figure 1. 
Study Theoretical Framework

\section{BEHAVIORAL FACTORS \\ Skills, Practice \\ (HIV Risk Behaviors)}

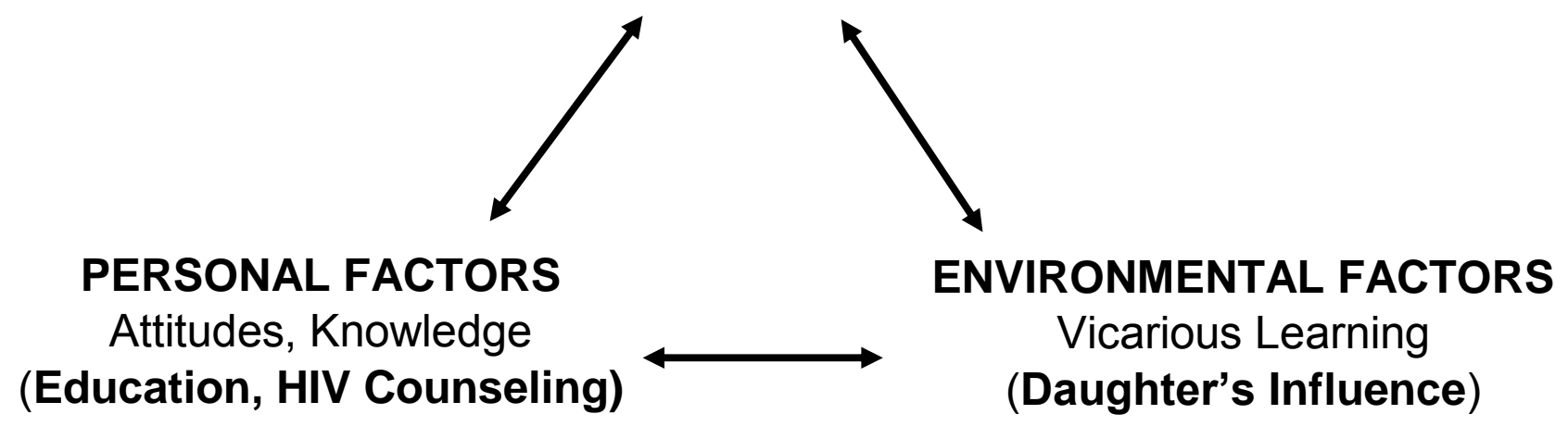

Based on Albert Bandura Social Cognitive Theoretical Model 
Figure 2.

Study Research Model

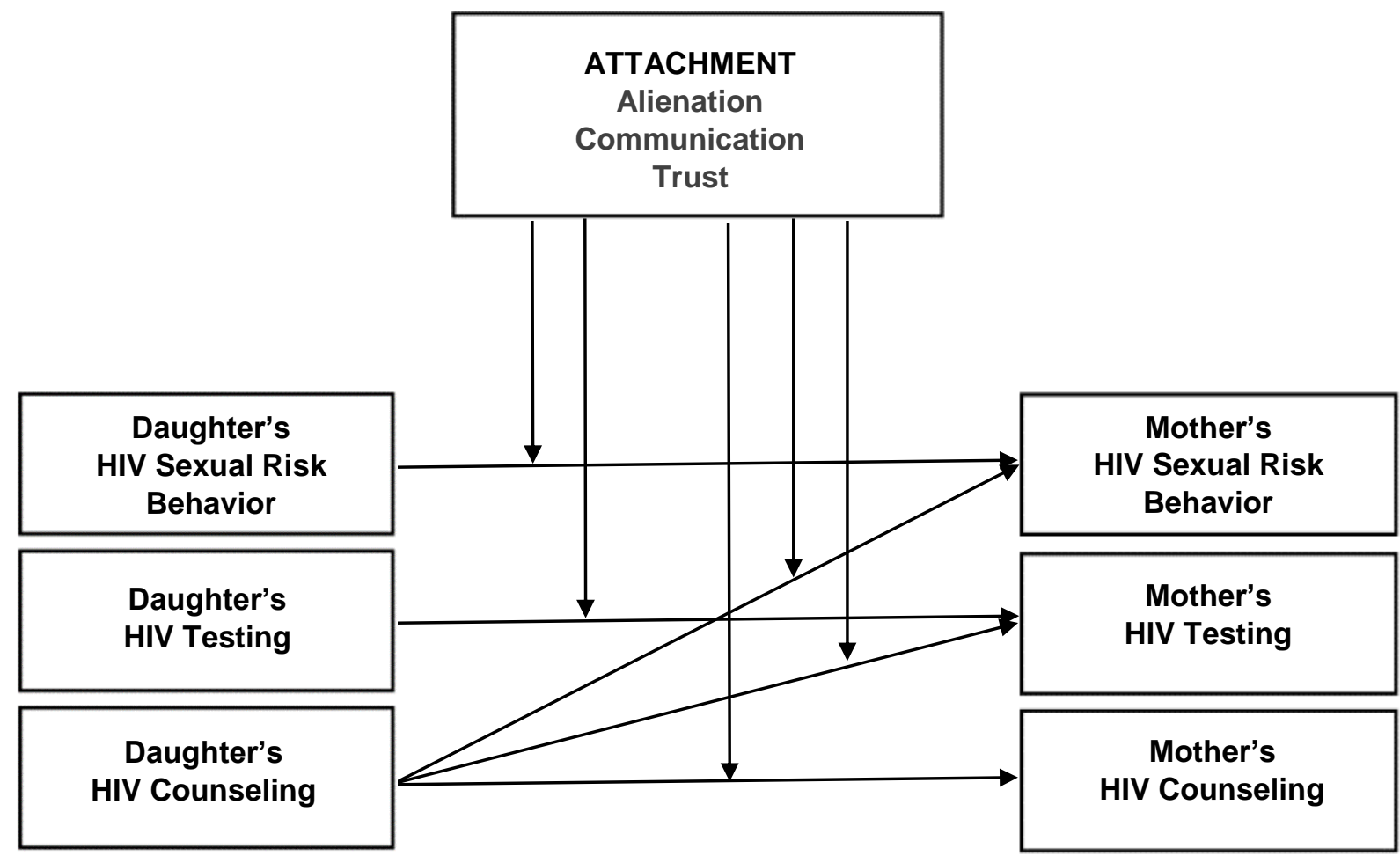


Figure 3.

Parent Study Longitudinal Timeframe

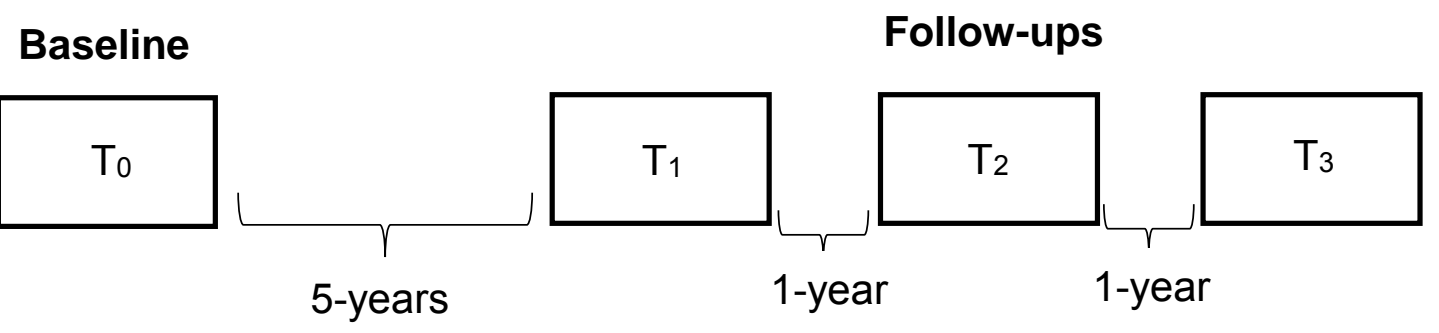

Figure 4.

PROCESS Conceptual Model

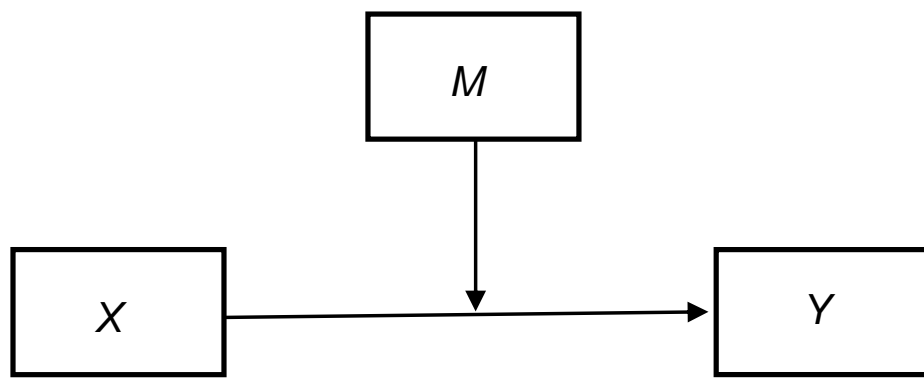

$$
\hat{Y}=i_{Y}+b_{1} X+b_{2} W+b_{3} X W \text {, }
$$


Figure 5.

$T_{0}$ : Recurrent Cross-sectional Moderator Model
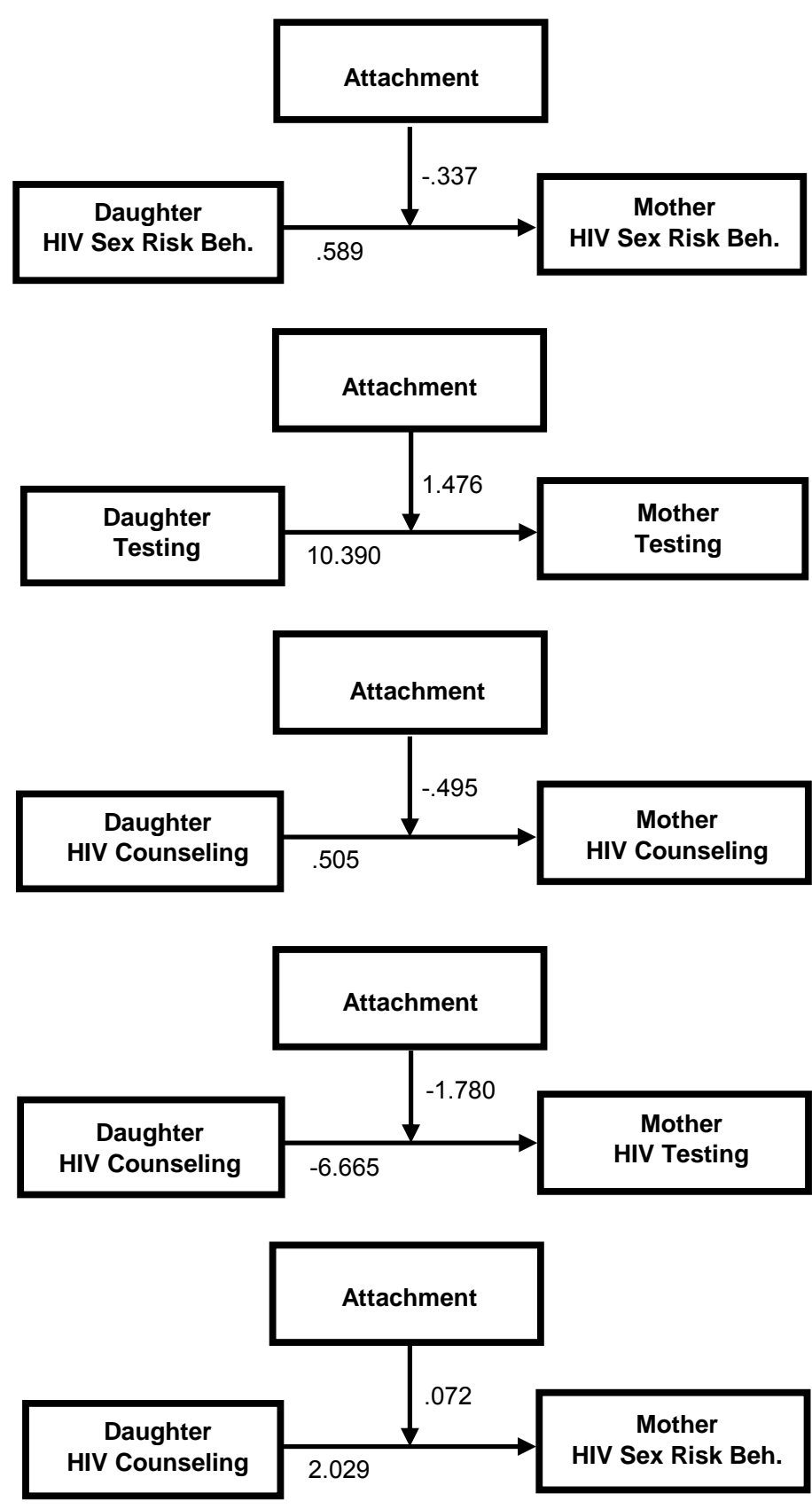

Note. Parameters reported in unstandardized path coefficients 
Figure 6.

$\mathrm{T}_{1}$ : Recurrent Cross-sectional Moderator Model
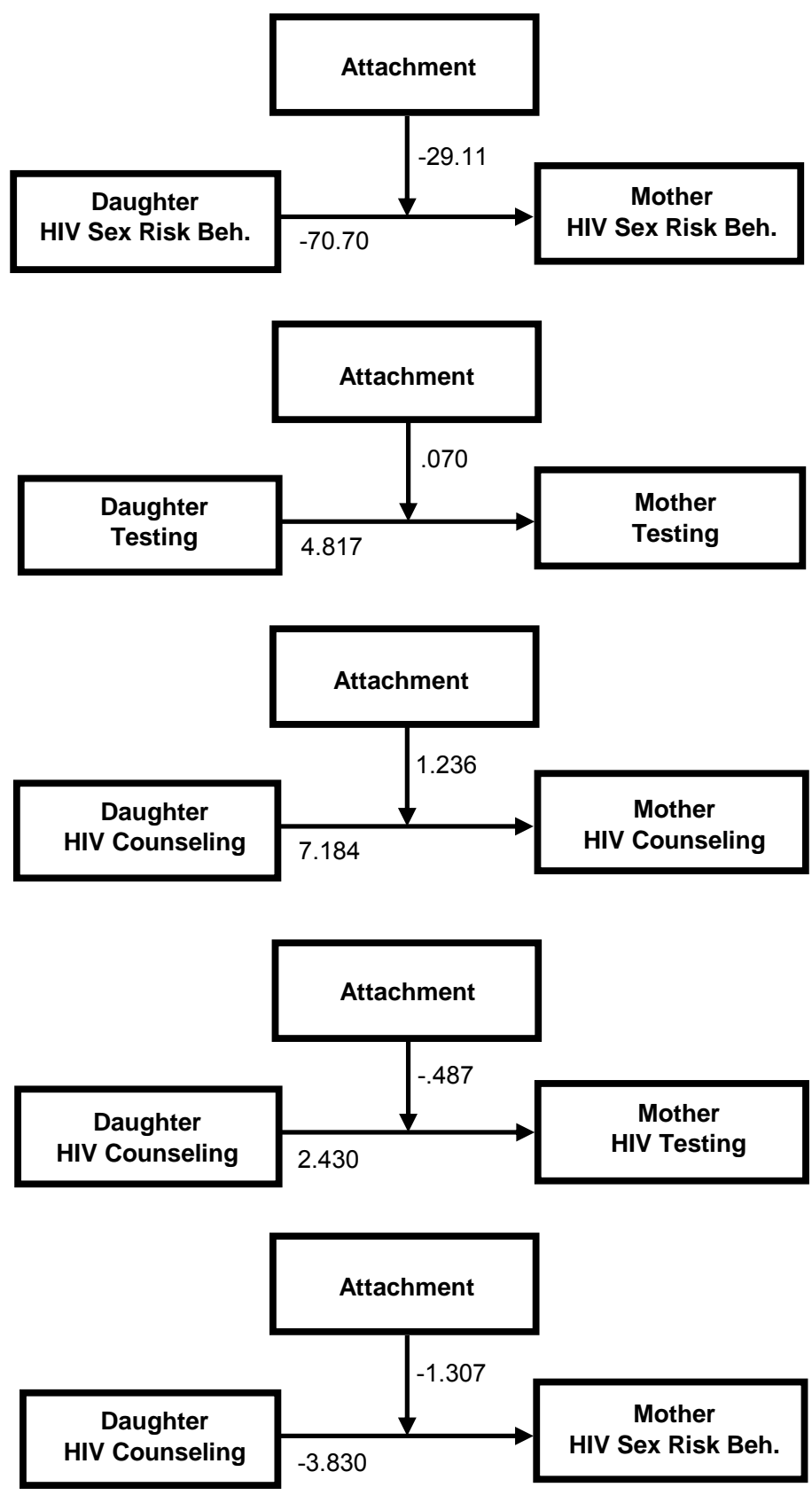

Note. Parameters reported in unstandardized path coefficients 
Figure 7.

$\mathrm{T}_{2}$ : Recurrent Cross-sectional Moderator Model
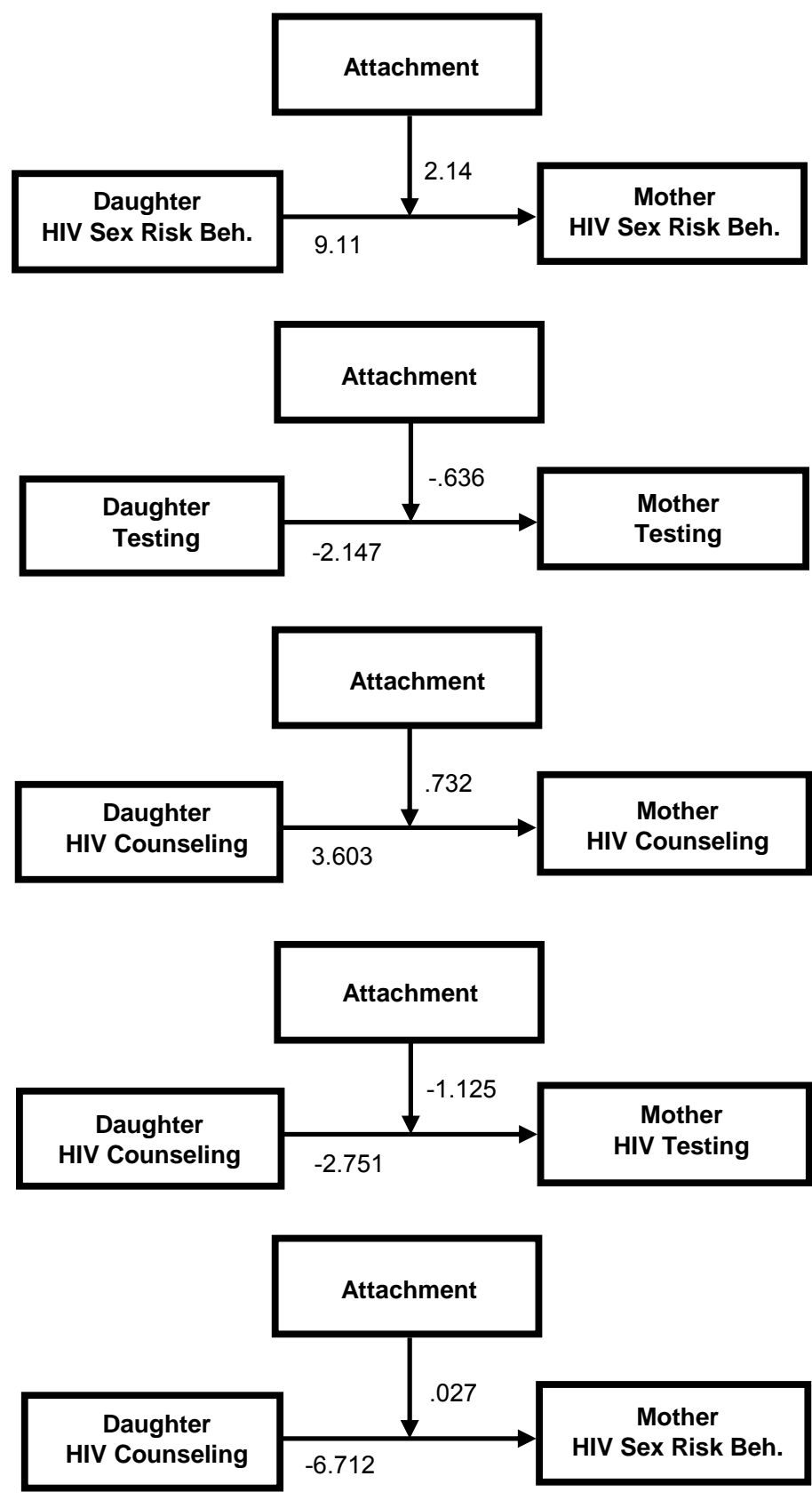

Note. Parameters reported in unstandardized path coefficients 
Figure 8.

$T_{3}$ : Recurrent Cross-sectional Moderator Model
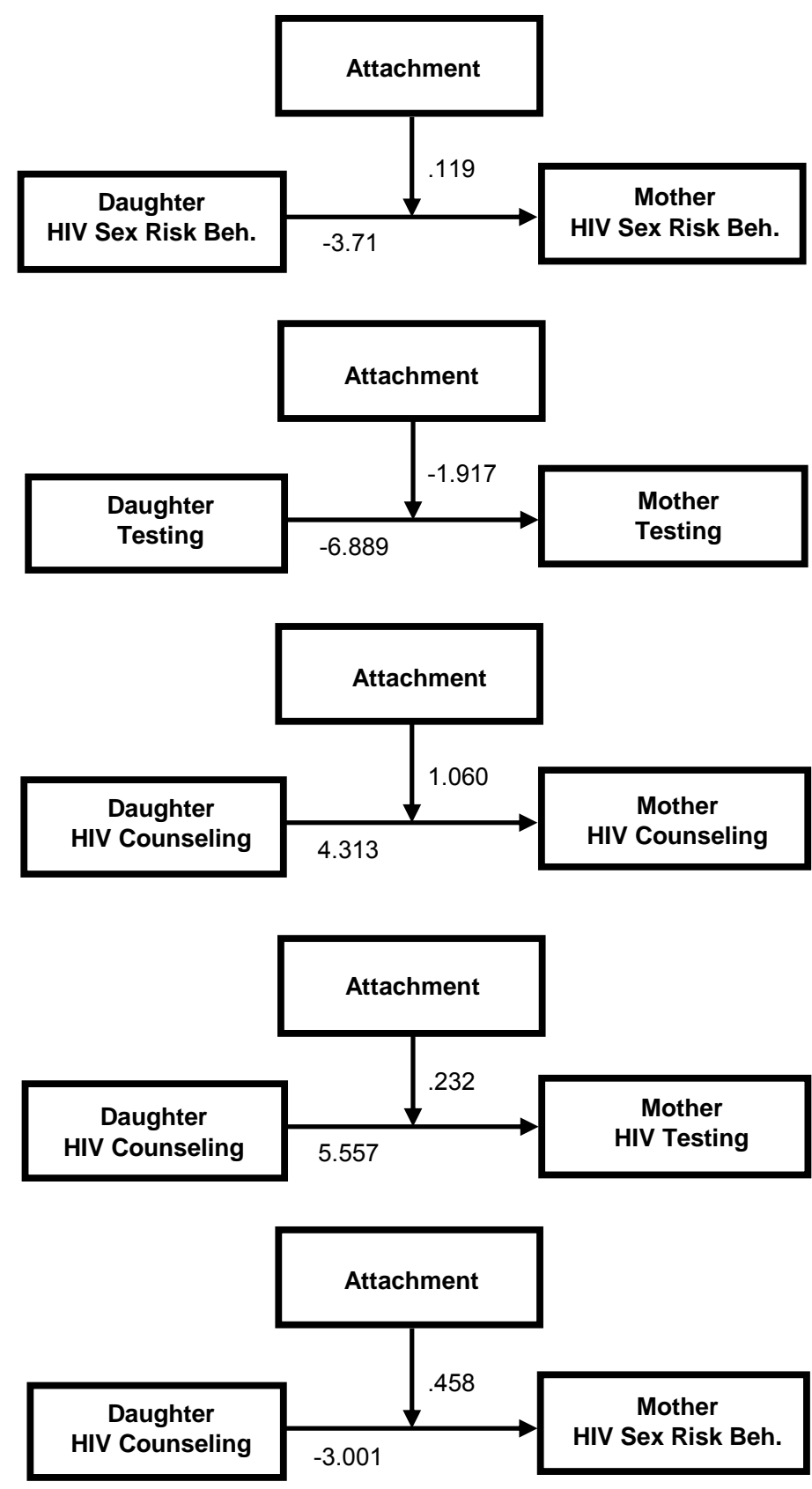

Note. Parameters reported in unstandardized path coefficients 


\section{References}

Abraído-Lanza, A. F., Armbrister, A. N., Flórez, K. R., \& Aguirre, A. N. (2006). Toward a theory-driven model of acculturation in public health research. American Journal of Public Health, 96(8), 1342-1346.

Adam, E. K., \& Chase-Lansdale, P. L. (2002). Home sweet home (s): parental separations, residential moves, and adjustment problems in low-income adolescent girls. Developmental psychology, 38(5), 792.

Adekeye, O. A., Heiman, H. J., Onyeabor, O. S., \& Hyacinth, H. I. (2012). The new invincibles: HIV screening among older adults in the US. PLOS One, 7(8), e43618.

Adimora, A. A., Schoenbach, V. J., Taylor, E. M., Khan, M. R., \& Schwartz, R. J. (2011). Concurrent partnerships, nonmonogamous partners, and substance use among women in the United States. American Journal of Public Health, 101(1), 128-136.

Adolph, C., Ramos, D. E. Linton, K. L., \& Grimes, D. A. (1995). Pregnancy among Hispanic teenagers: Is good parental communication a deterrent? Contraception, 51, 303-306.

Aiken, L. S., West, S. G., \& Reno, R. R. (1991). Multiple regression: Testing and interpreting interactions. Sage.

Ainsworth, M. (1973). The Development of Infant-mother Attachment [w:] B. Caldwell, HN Ricciuti (red.), Review of Child Development Research, 194.

Amaro, H., \& De la Torre, A. (2002). Public health needs and scientific opportunities in research on Latinas. American Journal of Public Health, 92(4), 525-529.

American Association of Retired Persons (AARP). (2014). Top 10 demographics $\&$ interests facts about American age $50+$. Retrieved from http://blog.aarp.org/2014/05/14/top-10-demographics-interests-factsabout-americans-age-50/

Andreeva, V. A., Unger, J. B., Yaroch, A. L., Cockburn, M. G., BaezcondeGarbanati, L., \& Reynolds, K. D. (2009). Acculturation and sun-safe behaviors among US Latinos: findings from the 2005 Health Information National Trends Survey. American Journal of Public Health, 99(4), 734741. 
Antshel, K. M. (2002). Integrating culture as a means of improving treatment adherence in the Latino population. Psychology, Health and Medicine, $7(4), 435-449$

Armsden, G. C., \& Greenberg, M. T. (1987). The inventory of parent and peer attachment: Individual differences and their relationship to psychological well-being in adolescence. Journal of Youth and Adolescence, 16(5), 427454.

Asch, S., Leake, B., \& Gelberg, L. (1994). Does fear of immigration authorities deter tuberculosis patients from seeking care? Western Journal of Medicine, 161(4), 373.

Ayanian, J. Z., Weissman, J. S., Schneider,E. C., Ginsburg, J. A., \& Zaslavsky, M. (2000). Unmet health needs of uninsured adults in the United States. Journal of American Medical Association, 284(16), 20612069.doi:10.1001/jama.284.16.2061

Baliunas, D., Rehm, J., Irving, H., \& Shuper, P. (2010). Alcohol consumption and risk of incident human immunodeficiency virus infection: a metaanalysis. International Journal of Public health, 55(3), 159-166.

Balogun, A. S. (2010). Islamic perspectives on HIVIAIDS and antiretroviral treatment: the case of Nigeria. African Journal of AIDS research, 9(4), 459-466.

Bandura, A. (1977). Social Learning Theory. Englewood Cliffs, NJ: Prentice-Hall.

Bandura, A. (1997). Self-efficacy: The exercise of control. New York: Freeman.

Bandura, A. (2001). Social cognitive theory: An agentic perspective. Annual Review of Psychology, 52(1), 1-26.

Bandura, A. \& Walters, R.H. (1963). Social Learning and Personality Development. New York: Holt, Rinehart \& Winston.

Baumeister, L. M., Flores, E., \& Marin, B. V. (1995). Sex information given to Latinas adolescents by parents. Health Education Research, 10, 233-239.

Bazell, R. (2007, November 6). Research rewrites first chapter of AIDS in U.S. msn.com. Retrieved from http://www.msnbc.msn.com/id/21653369/ ns/health-second_opinion/t/research-rewrites-first-chapter-aidsus/\#.UHIb403AeSo 
Beaulaurier, R. L., Craig, S. L., \& De La Rosa, M. (2009). Older Latinas and HIVIAIDS: An examination of sexuality and culture as they relate to risk and protective factors. Journal of Gerontological Social Work, 52(1), 4863.

Benavides, R., Bonazzo, C., \& Torres, R. (2006). Parent-child communication: A model for Hispanics on HIV prevention. Journal of Community Health Nursing, 23(2), 81-94.

Béné, C., \& Merten, S. (2008). Women and fish-for-sex: transactional sex, HIVIAIDS and gender in African fisheries. World Development, 36(5), 875899.

Berger Cardoso, J., Ren, Y., Swank, P., Sanchez, M., \& De La Rosa, M. (2016). Pre-to post-immigration sexual risk behaviour and alcohol use among recent Latino immigrants in Miami. Culture, Health \& Sexuality, 1-15.

Berk, M. L., \& Schur, C. L. (2001). The effect of fear on access to care among undocumented Latino immigrants. Journal of Immigrant Health, 3(3), 151156.

Billings, A. G., Cronkite, R. C., \& Moos, R. H. (1983). Social-environmental factors in unipolar depression: comparisons of depressed patients and nondepressed controls. Journal of Abnormal Psychology, 92(2), 119.

Black, K. A., \& McCartney, K. (1997). Adolescent Females' Security with Parents Predicts the Quality of Peer Interaction. Social Development, 6(1), 91-110.

Boffey, P. (1985). Reagan defends financing for AIDS. The New York Times. Retrieved from http://www.nytimes.com/1985/09/18/us/reagan-defendsfinancing-for- aids.html

Bowlby, J. (1969). Attachment, Vol. 1 of Attachment and loss.

Robert Wood Johnson Foundation, Braveman, P., \& Egerter, S. (2008). Overcoming obstacles to health: report from the Robert Wood Johnson Foundation to the Commission to Build a Healthier America. Robert Wood Johnson Foundation.

Broadhead, R. S., \& Heckathorn, D. D. (1994). AIDS prevention outreach among injection drug users: Agency problems and new approaches. Social Drug Problems, 41(3), 473-495. 
Broadhead, R. S., Heckathorn, D. D., Grund, J. P. C., Stern, L. S., \& Anthony, D. L. (1995). Drug users versus outreach workers in combating AIDS: preliminary results of a peer-driven intervention. Journal of Drug Issues, 25(3), 531-564.

Brooks, R. A., Etzel, M. A., Hinojos, E., Henry, C. L., \& Perez, M. (2005). Preventing HIV among Latino and African American gay and bisexual men in a context of HIV-related stigma, discrimination, and homophobia: perspectives of providers. AIDS Patient Care \& STDs, 19(11), 737-744.

Brown, L., Macintyre, K., \& Trujillo, L. (2003). Interventions to reduce HIVIAIDS stigma: what have we learned?. AIDS education and prevention, 15(1), 49-69.

Browne, J. L., \& Chan, A. Y. (2012). Using the Theory of Planned Behaviour and implementation intentions to predict and facilitate upward family communication about mammography. Psychology \& Health, 27(6), 655673.

Bustamante, A. V., Chen, J., Fang, H., Rizzo, J. A., \& Ortega, A. N. (2014). Identifying health insurance predictors and the main reported reasons for being uninsured among US immigrants by legal authorization status. The International Journal of Health Planning and Management, 29(1).

Bustamante, A. V., Fang, H., Garza, J., Carter-Pokras, O., Wallace, S. P., Rizzo, J. A., \& Ortega, A. N. (2012). Variations in healthcare access and utilization among Mexican immigrants: the role of documentation status. Journal of Immigrant and Minority Health, 14(1), 146-155.

Caetano, R., Vaeth, P. A., Ramisetty-Mikler, S., \& Rodriguez, L. A. (2009). The Hispanic Americans baseline alcohol survey: Alcoholic beverage preference across Hispanic national groups. Alcoholism: Clinical and Experimental Research, 33(1), 150-159.

Cahill, S., \& Valadéz, R. (2013). Growing older with HIVIAIDS: New public health challenges. American Journal of Public Health, 103(3), e7-e15.

Calzada, E. J., Fernandez, Y., \& Cortes, D. E. (2010). Incorporating the cultural value of respeto into a framework of Latino parenting. Cultural Diversity \& Ethnic Minority Psychology, 16(1), 77-86. doi:http://dx.doi.org.ezproxy.fiu. edu/10.1037/a0016071

Carballo-Dieguez, A., \& Dolezal, C. (1996). HIV risk behaviors and obstacles to condom use among Puerto Rican men in New York City who have sex with men. American Journal of Public Health, 86(11), 1619-1622. 
Castilla, J., Sobrino, P., de la Fuente, L., Noguer, I., Guerra, L., \& Parras, F. (2002). Late diagnosis of HIV infection in the era of highly active antiretroviral therapy: consequences for AIDS incidence. Aids, 16(14), 1945-1951.

Castillo-Mancilla, J., Allshouse, A., Collins, C., Hastings-Tolsma, M., Campbell, T. B., \& MaWhinney, S. (2012). Differences in Sexual Risk Behavior and HIVIAIDS Risk Factors Among Foreign-Born and US-Born Hispanic Women. Journal of Immigrant and Minority Health/Center for Minority Public Health, 14(1), 89-99. http://doi.org/10.1007/s10903-011-9529-7

Castro, F. G., Garfinkle, J., Naranjo, D., Rollins, M., Brook, J. S., \& Brook, D. W. (2007). Cultural traditions as "protective factors" among Latino children of illicit drug users. Substance Use and Misuse, 42(4), 621-642.

Catania, J. A., Coates, T., Kegeles, S., Fullilove, M. T., Peterson, J., Marin, B., ... \& Hulley, S. (1992). Condom use in multi-ethnic neighborhoods of San Francisco: the population-based AMEN (AIDS in Multi-Ethnic Neighborhoods) Study. American Journal of Public Health, 82(2), 284-287.

Centers for Disease Control and Prevention (CDC). (2003). Late versus early testing of HIV-16 sites, United States, 2000-2003. Retrieved from http://www.cdc.gov/mmwr/preview/mmwrhtml/mm5225a2.htm

Centers for Disease Control and Prevention (CDC. 2006). Revised recommendations for HIV testing of adults, adolescents, and pregnant women in health-care settings. MMWR. Morbidity and Mortality Weekly Report, 55(RR14), 1-17.

Centers for Disease Control and Prevention (CDC). (2013). Condom Fact Sheet in Brief. Retrieved from http://www.cdc.gov/condomeffectiveness/brief.html

Center for Disease Prevention and Control (CDC). (2015a). HIV among Hispanics/Latinos. Retrieved from http://www.cdc.gov/hiv/group/ racialethnic/hispanic latinos/index.html

Center for Disease Prevention and Control (CDC). (2015b). HIV surveillance report: Diagnosis of HIV infection in the United States and dependent areas, 2013. Retrieved from https://www.cdc.gov/hiv/pdf/library/ reports/surveillance/cdc-hiv-surveillance-report-2013-vol-25.pdf

Center for Disease Prevention and Control (CDC). (2015c). Communities in crisis: Is there a generalized HIV epidemic in impoverished urban areas of the United States. Retrieved from http://www.cdc.gov/hiv/group/ poverty.html 
Centers for Disease Control and Prevention (CDC). (2016). Mortality among centenarians in the United States, 2000-2014. Retrieved from https://www.cdc.gov/nchs/data/databriefs/db233.pdf

Centers for Disease Control and Prevention (CDC). (2017a). HIV among Latinos. Retrieved from https://www.cdc.gov/nchhstp/newsroom/docs/factsheets/ cdc-hiv-latinos-508.pdf

Centers for Disease Control and Prevention (CDC). (2017b). HIV among Hispanics/Latinos. Retrieved from https://www.cdc.gov/hiv/group/ racialethnic/hispaniclatinos/index.html

Centers for Disease Control and Prevention (CDC). (2017c). HIV among People aged 50 and over. Retrieved from https://www.cdc.gov/hiv/group/age/ olderamericans/index.html

Center for Disease Prevention and Control (CDC). (2017d). Communities in crisis: Is there a generalized HIV epidemic in impoverished urban areas of the United States? Retrieved from https://www.cdc.gov/hiv/group/ poverty.html

Cianelli, R., Ferrer, L., \& McElmurry, B. J. (2008). HIV prevention and low-income Chilean women: machismo, marianismo and HIV misconceptions. Culture, Health \& Sexuality, 10(3), 297-306.

Cianelli, R., Villegas, N., Lawson, S., Ferrer, L., Kaelber, L., Peragallo, N., \& Yaya, A. (2013). Unique factors that place older Hispanic women at risk for HIV: Intimate partner violence, machismo, and marianismo. Journal of the Association of Nurses in AIDS Care, 24(4), 341-354.

Ciechanowski, P., \& Katon, W. J. (2006). The interpersonal experience of health care through the eyes of patients with diabetes. Social Science \& Medicine, 63(12), 3067-3079.

Cohn, S. E., \& Clark, R. A. (2003). Sexually transmitted diseases, HIV, and AIDS in women. Medical Clinics of North America, 87(5), 971-995.

Cohen, J., Cohen, P., West, S. G., \& Aiken, L. S. (2003). Applied multiple correlation/regression analysis for the behavioral sciences. UK: Taylor \& Francis.

Coleman, C. L. (2003). Transmission of HIV infection among older adults: A population at risk. Journal of the Association of Nurses in AIDS Care, 14(1), 82-85. 
Cowgill, D. O. (1986). Aging around the world. Belmont, CA: Wadsworth

Craig, S. L. (2007). Correlates of voluntary HIV testing and counseling among middle aged and older Latinas. Florida International University.

Craig, S. L., Beaulaurier, R. L., Newman, F. L., De La Rosa, M., \& Brennan, D. J. (2012). Health and cultural determinants of voluntary HIV testing and counseling among middle-aged and older Latina women. Journal of Women \& Aging, 24(2), 97-112.

Cuellar, I., Arnold, B., \& Gonzalez, G. (1995). Cognitive referents of acculturation: assessment of cultural constructs in Mexican Americans. Journal of Community Psychology, 23(4), 339-356.

Cuellar, I., Arnold, B., \& Maldonado, R. (1995). Acculturation rating scale for Mexican Americans-II: A revision of the original ARSMA scale. Hispanic Journal of Behavioral Sciences, 17(3), 275-304.

Cutler, D. M., \& Lleras-Muney, A. (2010). Understanding differences in health behaviors by education. Journal of Health Economics, 29(1), 1-28.

Dang, B. N., Giordano, T. P., \& Kim, J. H. (2012). Sociocultural and structural barriers to care among undocumented Latino immigrants with HIV infection. Journal of Immigrant and Minority Health, 14(1), 124-31. doi: 10.1007/s10903-011-9542-x

De Cock, K. M., \& Johnson, A. M. (1998). From exceptionalism to normalization: A reappraisal of attitudes and practice around HIV testing. British Medical Journal, 316(7127), 290.

De La Rosa, M. (2002). Acculturation and Latino adolescent drug abuse: A research agenda for the future. Journal of Drug Use and Issue, 37(4), 429456.

De La Rosa, M., Dillon, F. R., Ganapati, N. E., Rojas, P., Pinto, E., \& Prado, G. (2010b). Mother-daughter attachment and drug abuse among Latinas in the United States. Journal of Drug Issues, 40(2), 379-404

De La Rosa, M., Dillon, F. R., Rojas, P., Schwartz, S. J., \& Duan, R. (2010a). Latina Mother-daughter dyads: Relations between attachment and sexual behavior under the influence of alcohol or drugs. Archives of Sexual Behavior, 39(6), 1305-1319. 
De La Rosa, M., Huang, H., Brook, J. S., Sanchez, M., Rojas, P., Kanamori, M., ... \& Martinez, M. (2016). Sociocultural determinants of substance misuse among adult Latinas of Caribbean and South and Central American descent: A longitudinal study of a community-based sample. Journal of Ethnicity in Substance Abuse, 1-21.

De La Rosa, M., Huang, H., Rojas, P., Dillon, F. R., Lopez-Quintero, C., Li, T., \& Ravelo, G. J. (2015). Influence of Mother-Daughter Attachment on Substance Use: A Longitudinal Study of a Latina Community-Based Sample. Journal of Studies on Alcohol and Drugs, 76(2), 307-316.

De La Rosa, M., Vega, R., \& Radisch, M. A. (2000). The role of acculturation in the substance abuse behavior of African-American and Latino adolescents: Advances, issues, and recommendations. Journal of Psychoactive Drugs, 32(1), 33-42.

Delgado-Gaitan, C. (1994). Socializing young children in Mexican-American families: An intergenerational perspective. In P. Greenfield \& R. Cocking (Eds.), Cross-cultural roots of minority child development (pp. 55-86). Hillside, NJ: Erlbaum.

Derose, K. P., Escarce, J. J., \& Lurie, N. (2007). Immigrants and health care: sources of vulnerability. Health Affairs, 26(5), 1258-1268.

DiFranceisco, W., Pinkerton, S. D., Dyatlov, R. V., \& Swain, G. R. (2005). Evidence of a brief surge in safer sex practices after HIV testing among a sample of high risk men and women. JAIDS, 39(5), 606-612.

Dillon, F. R., Félix-Ortiz, M., Rice, C., De La Rosa, M., Rojas, P., \& Duan, R. (2009). Validating the Multidimensional Measure of Cultural Identity Scales for Latinos among Latina mothers and daughters. Cultural Diversity and Ethnic Minority Psychology, 15(2), 191.

Ditzen, B., Schmidt, S., Strauss, B., Nater, U. M., Ehlert, U., \& Heinrichs, M. (2008). Adult attachment and social support interact to reduce psychological but not cortisol responses to stress. Journal of Psychosomatic Research, 64(5), 479-486.

Dixon, S. V., Graber, J. A., \& Brooks-Gunn, J. (2008). The roles of respect for parental authority and parenting practices in parent-child conflict among African American, Latino, and European American families. Journal of Family Psychology, 22(1), 1. 
Edberg, M. (2015). Essentials of health behavior: Social and Behavioral Theory in Public Health (2 ${ }^{\text {nd }}$ Ed.). Boston, MA: Jones and Bartlett Publishers.

Edwards, L.M., Fehring, R.J., Jarrett, K.M., Haglund, K.A. (2008). The influence of religiosity, gender, and language preference acculturation on sexual activity among Latino/a adolescents. Hispanic Journal of Behavioral Sciences, 30, 447-462. doi: 10.1177/0739986308322912

Effros, R. B., Fletcher, C. V., Gebo, K., Halter, J. B., Hazzard, W. R., Horne, F. M., ... \& Nayfield, S. G. (2008). Workshop on HIV infection and aging: what is known and future research directions. Clinical infectious diseases: an official publication of the Infectious Diseases Society of America, 47(4), 542.

Elifson, K.W., Klein, H., \& Sterk, C.E. (2003). Religiosity and HIV risk behavior among at risk women. Journal of Religion and Health, 42, 47-66. doi:10.1023/A:1022264711670

Ellman, T. M., Sexton, M. E., Warshafsky, D., Sobieszczyk, M. E., \& Morrison, E. A. (2014). A forgotten population: older adults with newly diagnosed HIV. AIDS Patient Care and STDs, 28(10), 530-536.

Emlet, C. A., \& Farkas, K. J. (2001). A descriptive analysis of older adults with HIVIAIDS in California. Health \& social work, 26(4), 226-234.

Falvo, N., \& Norman, S. (2004). Never too old to learn: The impact of an HIVIAIDS education program on older adults' knowledge. Clinical Gerontologist, 27(1-2), 103-117.

Felix-Ortiz, M., Newcomb, M. D., \& Myers, H. (1994). A multidimensional measure of cultural identity for Latino and Latina adolescents. Hispanic Journal of Behavioral Sciences, 16(2), 99-115.

Fergusson, D. M., Boden, J. M., \& Horwood, L. J. (2007). Recurrence of major depression in adolescence and early adulthood, and later mental health, educational and economic outcomes. The British Journal of Psychiatry, 191(4), 335-342.

Ferrari, A. M. (2002). The impact of culture upon child rearing practices and definitions of maltreatment. Child Abuse \& Neglect, 26(8), 793-813.

Field, A. (2013). Discovering statistics using IBM SPSS statistics. Sage. 
Fingerman, K. L., Chen, P. C., Hay, E., Cichy, K. E., \& Lefkowitz, E. S. (2006). Ambivalent reactions in the parent and offspring relationship. The Journals of Gerontology Series B: Psychological Sciences and Social Sciences, 61(3), P152-P160.

Fisher, M. (2008). Late diagnosis of HIV infection: major consequences and missed opportunities. Current Opinion in Infectious Diseases, 21(1), 1-3.

Flores, G. (2000). Culture and the patient-physician relationship: achieving cultural competency in health care. The Journal of pediatrics, 136(1), 1423.

Flores, A. (2017). How the U.S. Hispanic population is changing. Pew Research Center. Retrieved from http://www.pewresearch.org/fact-tank/2017/09/18/ how-the-u-s-hispanic-population-is-changing/

Florez, K. R., Derose, K. P., Breslau, J., Griffin, B. A., Haas, A. C., Kanouse, D. E., ... Williams, M. V. (2015). Acculturation and drug use stigma among Latinos and African Americans: An examination of a church-based sample. Journal of Immigrant and Minority Health, 17(6), 1607-1614

Formoso, D., Gonzales, N. A., \& Aiken, L. S. (2000). Family conflict and children's internalizing and externalizing behavior: Protective factors. American Journal of Community Psychology, 28(2), 175-199.

Francesca, E. (2002). AIDS in contemporary Islamic ethical literature. Medicine and Law, 21, 381.

Freeman, R. C., Lewis, Y. P., \& Colon, H. M. (2002). Handbook for conducting drug abuse research with Hispanic populations. Westport, CT: Praeger

Gaeta, T. J., LaPolla, C., \& Melendez, E. (1996). AIDS in the elderly: New York City vital statistics. The Journal of Emergency Medicine, 14(1), 19-23.

Galanti, G. A. (2003). The Hispanic family and male-female relationships: An overview. Journal of Transcultural Nursing, 14(3), 180-185.

Gil, A. G., Wagner, E. F., \& Vega, W. A. (2000). Acculturation, feminism, and alcohol use among Latino adolescent males: longitudinal relations. Journal of Community Psychology, 28(4), 443-458.

Gill, R. M., \& Vazquez, C. I. (1996). The Maria paradox. New York: Putnam's Sons. 
Gilbert, P. A., \& Rhodes, S. D. (2013). HIV testing among immigrant sexual and gender minority Latinos in a US region with little historical Latino presence. AIDS Patient Care and STDs, 27(11), 628-636.

Glanz, K., Rimer, B.K. \& Lewis, F.M. (2002). Health Behavior and Health Education. Theory, Research and Practice. San Francisco: Wiley \& Sons.

Golub, S. A., Tomassilli, J. C., Pantalone, D. W., Brennan, M., Karpiak, S. E., \& Parsons, J. T. (2010). Prevalence and correlates of sexual behavior and risk management among HIV-positive adults over 50. Sexually Transmitted Diseases, 37(10), 615-620.

Gómez, C. A., \& Marin, B. V. (1996). Gender, culture, and power: Barriers to HIV-prevention strategies for women. Journal of Sex Research, 33(4), 355-362.

Gonzalez, J. S., Hendriksen, E. S., Collins, E. M., Durán, R. E., \& Safren, S. A. (2009). Latinos and HIVIAIDS: examining factors related to disparity and identifying opportunities for psychosocial intervention research. AIDS and Behavior, 13(3), 582.

González-Guarda, R. M., Peragallo, N., Urrutia, M. T., Vasquez, E. P., \& Mitrani, V. B. (2008). HIV risks, substance abuse, and intimate partner violence among Hispanic women and their intimate partners. Journal of the Association of Nurses in AIDS Care, 19(4), 252-266.

Gonzalez-Ramos, G., Zayas, L. H., \& Cohen, E. V. (1998). Child-rearing values of low-income, urban Puerto Rican mothers of preschool children. Professional Psychology: Research \& Practice, 29, 377-382.

Graham, M. A., Tomar, S. L., \& Logan, H. L. (2005). Perceived social status, language and identified dental home among Hispanics in Florida. The Journal of the American Dental Association, 136(11), 1572-1582.

Grossoehme, D., \& Lipstein, E. (2016). Analyzing longitudinal qualitative data: the application of trajectory and recurrent cross-sectional approaches. BMC Research Notes, 9(1), 136.

Guo, Y., \& Sims, O. T. (2017). Assessment of recent HIV testing among older adults in the United States. Social Work in Health Care, 56(9), 855-864.

Hair, J.F., Black, B., Babin, B., Anderson, R.E., \& Tatham, R.L. (2006). Multivariate Data Analysis, Sixth Edition. Pearson Prentice Hall: Upper Saddle River, NJ. 
Halgunseth, L. C., Ispa, J. M., \& Rudy, D. (2006). Parental control in Latino families: an integrated review of the literature. Child Development, 77(5), 1282-1297.

Harding, J. R., \& Manry, J. (2017). Provider sexual health assessment of the aging adult. Educational Gerontology, 1-6.

Harris, C. M., McKenzie, R., Nayak, S., Kiyatkin, D., Baker, D., \& Kisuule, F. (2015). Graying of the HIV epidemic: a challenge for inpatient medicine providers. Journal of Community Hospital Internal Medicine Perspectives, 5(6), 10.3402/jchimp.v5.29428. http://doi.org/10.3402/jchimp.v5.29428

Harrison, K. M., Song, R., \& Zhang, X. (2010). Life expectancy after HIV diagnosis based on national HIV surveillance data from 25 states, United States. Journal of Acquired Immune Deficiency Syndromes, 53(1), 124130. doi: 10.1097/QAI.0b013e3181b563e7

Harvey, S. M., \& Henderson, J. T. (2006). Correlates of condom use intentions and behaviors among a community-based sample of Latino men in Los Angeles. Journal of Urban Health, 83(4), 558-574.

Harwood, R. L., Miller, J. G., \& Irizarry, N. L. (1995). Culture and attachment: Perceptions of the child in context. New York: Guilford Press.

Hayes, A. F. (n.d.). The PROCESS macro for SPSS and SAS. Retrieved from http://www.processmacro.org/index.html

Hayes, A. F. (2012). PROCESS: A versatile computational tool for observed variable mediation, moderation, and conditional process modeling [White paper]. Retrieved from http://www.afhayes.com/public/process2012.pdf

Heckathorn, D. D. (1997). Respondent-driven sampling: a new approach to the study of hidden populations. Social problems, 44(2), 174-199.

Heckathorn, D. D. (2011). Snowball versus respondent-driven sampling. Sociological Methodology, 41(1), 355-366. doi.org/10.1111/ j.1467-9531.2011.01244.x

Heckathorn, D. D., \& Broadhead, R. S. (1996). Rational choice, public policy and AIDS. Rationality and Society, 8(3), 235-260.

Hedeker, D., Gibbons, R. D., \& Waternaux, C. (1999). Sample size estimation for longitudinal designs with attrition: comparing time-related contrasts between two groups. Journal of Educational and Behavioral Statistics, 24(1), 70-93. 
Heller, P. L. (1970). Familism scale: A measure of family solidarity. Journal of Marriage and the Family, 73-80.

Henao-Martínez, A. F., \& Castillo-Mancilla, J. R. (2013). The Hispanic HIV epidemic. Current infectious disease reports, 15(1), 46-51.

Hill, P. C., \& Pargament, K. I. (2003). Advances in the conceptualization and measurement of religion and spirituality: Implications for physical and mental health research. American Psychologist, 58, 64-74.

Hillman, J. (2008). Knowledge, attitudes, and experience regarding HIVIAIDS among older adult inner-city Latinos. International Journal of Aging \& Human Development, 66(3), 243-257. doi: 10.2190/AG.66.3.e

Hines, A. M., \& Caetano, R. (1998). Alcohol and AIDS-related sexual behavior among Hispanics: Acculturation and gender differences. AIDS Education and Prevention, 10(6), 533.

HIV-Causal Collaboration. (2010). The effect of combined antiretroviral therapy on the overall mortality of HIV-infected individuals. AIDS (London, England), 24(1), 123.

Hovell, M., Sipan, C. Blumberg, E., Atkins, C., Hofsteter, C. R., \& Kreitner, S. (1994). Family influences on Latino and Anglo adolescents' sexual behavior. Journal of Marriage and the Family, 56, 973-986.

Huey Jr, S. J., Tilley, J. L., Jones, E. O., \& Smith, C. A. (2014). The contribution of cultural competence to evidence-based care for ethnically diverse populations. Annual Review of Clinical Psychology, 10, 305-338.

Hughes, A. K. (2011). HIV Knowledge and Attitudes among Providers in Aging: Results from a National Survey. AIDS Patient Care and STDs, 25(9), 539545. doi:10.1089/apc.2011.0026

Hunt, L. M., Schneider, S., \& Comer, B. (2004). Should "acculturation" be a variable in health research? A critical review of research on US Hispanics. Social Science \& Medicine, 59(5), 973-986.

Hutchinson, M. K., Jemmott, J. B., Jemmott, L. S., Braverman, P., \& Fong, G. T. (2003). The role of mother-daughter sexual risk communication in reducing sexual risk behaviors among urban adolescent females: a prospective study. Journal of Adolescent Health, 33(2), 98-107. 
Ickovics, J. R., Beren, S. E., Grigorenko, E. L., Morrill, A. C., Druley, J. A., \& Rodin, J. (2002). Pathways of risk: Race, social class, stress, and coping as factors predicting heterosexual risk behaviors for HIV among women. AIDS and Behavior, 6(4), 339-350.

Idris A. M., Elsamani E. Z., Elnasri A. A. (2015). Sociodemographic predictors of acceptance of voluntary HIV testing among pregnant women in a large maternity hospital, Omdurman, Sudan. Eastern Mediterranean Health Journal, 21, 273-279.

Infectious Diseases Society of America. (2008, August 1). Higher HIV Infection Estimate Shows Need for Routine Screening, More Funding for Care. ScienceDaily. Retrieved from http://www.sciencedaily.com-/releases/ 2008/08/080801152149.htm

Inungu, J. N., Mokotoff, E. D., \& Kent, J. B. (2001). Characteristics of HIV infection in patients fifty years or older in Michigan. AIDS Patient Care and STDs, 15(11), 567-573.

Jaccard, J., Wan, C. K., \& Turrisi, R. (1990). The detection and interpretation of interaction effects between continuous variables in multiple regression. Multivariate behavioral research, 25(4), 467-478.

Jacobs, E., A., Karavolos, K., Rathouz, P. J., Ferris, T. G., \& Powell, L. H. (2005). Limited English proficiency and breast and cervical cancer screening in a multiethnic population. American Journal of Public Health, 95(8), 14101416.

Jacobs, R. J., \& Thomlison, B. (2009). Self-silencing and age as risk factors for sexually acquired HIV in midlife and older women. Journal of Aging and Health, 21(1), 102-128.

Jarama, S. L., Belgrave, F. Z., Bradford, J., Young, M., \& Honnold, J. A. (2007). Family, cultural and gender role aspects in the context of HIV risk among African American women of unidentified HIV status: An exploratory qualitative study. AIDS care, 19(3), 307-317.

Jena, A. B., Goldman, D. P., Kamdar, A., Lakdawalla, D. N., \& Lu, Y. (2010). Sexually transmitted diseases among users of erectile dysfunction drugs. Annals of Internal Medicine, 153(1), 1-7. http://doi.org/10.1059/00034819-153-1-201007060-00003

Johnson, B. K. (2013). Sexually transmitted infections and older adults. Journal of Gerontological Nursing, 39(11), 53-60. 
Joint United Nations Programme on HIVIAIDS (UNAIDS). (2003). Fact

Sheet: Stigma and discrimination. Retrieved from http://data.unaids.org/ publications/Fact-Sheets03/fs_stigma_discrimination_en.pdf

Joint United Nations Programme on HIVIAIDS (UNAIDS). (2008). Report on the Global Acquired Immunodeficiency Syndrome Epidemic. Retrieved from http://www.unaids.org/sites/default/files/media_asset/UNAIDS_Global_Re port_2013_en_1.pdf

Kaiser Family Foundation (KFF). (2012). The global HIV/AIDS timeline. Retrieved from http://www.kff.org/hivaids/timeline/hivtimeline.cfm

Kaiser Family Foundation (KFF). (2014). Latinos and HIVIAIDS. Retrieved from https://www.kff.org/hivaids/fact-sheet/latinos-and-hivaids/\#footnote108079-2

Kalichman, S. C., Simbayi, L. C., Kaufman, M., Cain, D., \& Jooste, S. (2007). Alcohol use and sexual risks for HIVIAIDS in sub-Saharan Africa: systematic review of empirical findings. Prevention science, 8(2), 141-151.

Kaplan, C. P., Nápoles-Springer, A., Stewart, S. L., \& Pérez-Stable, E. J. (2001). Smoking acquisition among adolescents and young Latinas: The role of socioenvironmental and personal factors. Addictive Behaviors, 26(4), 53155.

Karpiak, S. E., Shippy, R. A., \& Cantor, M. H. (2006). ROAH: Research on older adults with HIV. New York: AIDS Community.

Kline, R. B. (1998). Methodology in the social sciences. Principles and practice of structural equation modeling. New York: Guilford Press

Knipper, E., Rhodes, S. D., Lindstrom, K., Bloom, F. R., Leichliter, J. S., \& Montaño, J. (2007). Condom use among heterosexual immigrant Latino men in the southeastern United States. AIDS Education and Prevention, 19(5), 436.

Kratzke, C., Amatya, A., \& Vilchis, H. (2014). Differences among college women for breast cancer prevention acquired information-seeking, desired apps and texts, and daughter-initiated information to mothers. Journal of Community Health, 39(2), 291-300.

Kratzke, C., Vilchis, H., \& Amatya, A. (2013). Breast cancer prevention knowledge, attitudes, and behaviors among college women and motherdaughter communication. Journal of Community Health, 38(3), 560-568. 
Krueger, L. E., Wood, R. W., Diehr, P. H., \& Maxwell, C. L. (1990). Poverty and HIV seropositivity: the poor are more likely to be infected. Aids, 4(8), 811814.

Lekas, H. M., Schrimshaw, E. W., \& Siegel, K. (2005). Pathways to HIV testing among adults aged fifty and older with HIVIAIDS. Aids Care, 17(6), 674687.

Liddicoat, R. V., Horton, N. J., Urban, R., Maier, E., Christiansen, D., \& Samet, J. $\mathrm{H}$. (2004). Assessing missed opportunities for HIV testing in medical settings. Journal of General Internal Medicine, 19(4), 349-356.

Liebowitz, S. W., Castellano, D. C., \& Cuellar, I. (1999). Factors that predict sexual behaviors among young Mexican American adolescents: An exploratory study. Hispanic Journal of Behavioral Sciences, 21(4), 470479. doi: $10.1177=0739986399214006$

Life Course and Health Research Center. (1997). Transitions: A study of stress and wellbeing in young adulthood. Wave I questionnaire. Title of project: Drug Use Trajectories-Ethnic/Racial Comparisons. NIDA Grant Number DA10772. Florida International University, Miami, FL. Retrieved from http://www.fiu.edu/ Ichrc/transitions.htm)

Lindau, S. T., Leitsch, S. A., Lundberg, K. L., \& Jerome, J. (2006). Older women's attitudes, behavior, and communication about sex and HIV: A community-based study. Journal of Women's Health, 15(60), 747-753.

Lindau, S. T., Schumm, P., Laumann, E. O., Levinson, W., O'Muircheartaigh, C. A., \& Waite, L. J. (2007). A study of sexuality and health among older adults in the United States. New England Journal of Medicine, 357, 762774. doi: 10.1056/NEJMoa067423

Linley, L., Prejean, J., An, Q., Chen, M., \& Hall, H. I. (2012). Racial/ethnic disparities in HIV diagnoses among persons aged 50 years and older in 37 US States, 2005-2008. American Journal of Public Health, 102(8), 1527-1534.

Linsk, N. L. (2000). HIV among older adults: age-specific issues in prevention and treatment. The AIDS Reader, 10(7), 430-440.

Little, T. D., Card, N. A., Bovaird, J. A., Preacher, K. J., \& Crandall, C. S. (2007). Structural equation modeling of mediation and moderation with contextual factors. Modeling Contextual Effects in Longitudinal Studies, 1, 207-230. 
Lopez-Quintero, C., Rojas, P., Dillon, F. R., Varga, L. M., \& De La Rosa, M. (2016). HIV testing practices among Latinas at risk of getting infected: a five-year follow-up of a community sample in South Florida. AIDS care, 28(2), 137-146. doi.org/10.1080/09540121.2015.1071769

Lopez-Quintero, C., Shtarkshall, R., \& Neumark, Y. D. (2005). Barriers to HIVtesting among Hispanics in the united states: Analysis of the national health interview survey, 2000. AIDS Patient Care and STDs, 19(10), 672683

Lorenc, A., Ananthavarathan, P., Lorigan, J., Jowata, M., Brook, G., \& Banarsee, R. (2014). The prevalence of comorbidities among people living with HIV in Brent: a diverse London Borough. London Journal of Primary Care, 6(4), 84-90.

Lorenz, K. (1935). Der Kumpan in der Umwelt des Vogels. Der Artgenosse als auslösendes Moment sozialer Verhaltensweisen. Journal für Ornithologie, 83, 137-215, 289-413.

Lubinski, C., Aberg, J., Bardeguez, A. D., Elion, R., Emmanuel, P., Kuritzkes, D., ... \& Zerehi, M. R. (2009). HIV policy: the path forward-a joint position paper of the HIV Medicine Association of the Infectious Diseases Society of America and the American College of Physicians. Clinical Infectious Diseases, 48(10), 1335-1344.

Mahajan, A. P., Sayles, J. N., Patel, V. A., Remien, R. H., Ortiz, D., Szekeres, G., \& Coates, T. J. (2008). Stigma in the HIVIAIDS epidemic: a review of the literature and recommendations for the way forward. AIDS (London, England), 22(2), S67.

Marin, B. V., \& Marin, G. (1992). Predictors of condom accessibility among Hispanics in San Francisco. American Journal of Public Health, 82(4), 592-595.

Marin, G., \& Marin, B. V. O. (1991). Research with Hispanic populations. Newbury Park, CA: Sage.

Marín, B. V., Tschann, J. M., Gómez, C. A., \& Gregorich, S. (1998). Self-efficacy to use condoms in unmarried Latino adults. American Journal of Community Psychology, 26(1), 53-71.

Marin, B. V., Tschann, J. M., Gomez, C. A., \& Kegeles, S. M. (1993). Acculturation and gender differences in sexual attitudes and behaviors: Hispanic vs non-Hispanic white unmarried adults. American Journal of Public Health, 83(12), 1759-1761. 
Marks, G., Crepaz, N., Senterfitt, J. W., \& Janssen, R. S. (2005). Meta-analysis of high-risk sexual behavior in persons aware and unaware they are infected with HIV in the United States: Implications for HIV prevention programs. Journal of Acquired Immune Deficiency Syndrome, 39(4), 446453.

Maunder, R. G., \& Hunter, J. J. (2001). Attachment and psychosomatic medicine: Developmental contributions to stress and disease. Psychosomatic Medicine, 63(4), 556-567.

Maunder, R. G., Lancee, W. J., Hunter, J. J., Greenberg, G. R., \& Steinhart, A. H. (2005). Attachment insecurity moderates the relationship between disease activity and depressive symptoms in ulcerative colitis. Inflammatory Bowel Diseases, 11(10), 919-926.

Maunder, R. G., Lancee, W. J., Nolan, R. P., Hunter, J. J., \& Tannenbaum, D. W. (2006). The relationship of attachment insecurity to subjective stress and autonomic function during standardized acute stress in healthy adults. Journal of Psychosomatic Research, 60(3), 283-290.

Mena, M. P., Dillon, F. R., Mason, C. A., \& Santisteban, D. A. (2008). Communication about sexually-related topics among Hispanic substanceabusing adolescents and their parents. Journal of Drug Issues, 38(1), 215234.

McCaig, L. F., Hardy, A. M., \& Winn, D. M. (1991). Knowledge about AIDS and HIV in the US adult population: influence of the local incidence of AIDS. American Journal of Public Health, 81(12), 1591-1595.

McGraw, L. A., \& Walker, A. J. (2004). Negotiating care: Ties between aging mothers and their caregiving daughters. The Journals of Gerontology Series B: Psychological Sciences and Social Sciences, 59(6), S324-S332.

McMahon, J., Wanke, C., Terrin, N., Skinner, S., \& Knox, T. (2011). Poverty, hunger, education, and residential status impact survival in HIV. AIDS and Behavior, 15(7), 1503-1511.

Meredith, P., Strong, J., \& Feeney, J. A. (2006). Adult attachment, anxiety, and pain self-efficacy as predictors of pain intensity and disability. Pain, 123(1), 146-154.

Monshipouri, M., \& Trapp, T. (2012). HIVIAIDS, religion, and human rights: a comparative analysis of Bangladesh, Indonesia, and Iran. Human Rights Review, 13(2), 187-204. 
Moreno, C. L. (2007). The relationship between culture, gender, structural factors, abuse, trauma, and HIVIAIDS for Latinas. Qualitative Health Research, 17(3), 340-352.

Mosavel, M. (2009). The feasibility of mothers accepting health advice from their adolescent daughters. Journal of Health Care for the Poor and Underserved, 20(1), 42-49

Mosavel, M., \& Genderson, M. W. (2016). Daughter-initiated cancer screening appeals to mothers. Journal of Cancer Education, 31(4), 767-775.

Mosavel, M., \& Thomas, T. (2009). Daughter-initiated health advice to mothers: Perceptions of African-American and Latina daughters. Health Education Research, 24(5), 799-810.

Mugavero, M. J., Castellano, C., Edelman, D., \& Hicks, C. (2007). Late diagnosis of HIV infection: The role of age and sex. American Journal of Medicine, 120, 370-373. doi:10.1016/j.amjmed.2006.05.050

Naimi, T. S., Brewer, R. D., Mokdad, A., Denny, C., Serdula, M. K., \& Marks, J. S. (2003). Binge drinking among US adults. JAMA, 289(1), 70-75.

National Association of Social Work (NASW). (2014). NASW HIVIAIDS Spectrum: Mental Health Training \& Education of Social Workers Project. Retrieved from https://www.socialworkers.org/LinkClick.aspx?fileticket= yzpg-m3BD3U\%3D\&portalid=0

National HIVIAIDS Strategy for the United States. (2010). Retrieved from https://www.whitehouse.gov/sites/default/files/uploads/NHAS.pdf

National HIVIAIDS Strategy for the United States. (2015). Retrieved from https://www.aids.gov/federal-resources/national-hiv-aids-strategy/ nhas-update.pdf

National Institute on Alcohol Abuse and Alcoholism (NIAAA). (n.d.). Drinking levels defined. Retrieved from https://www.niaaa.nih.gov/alcoholhealth/overview-alcohol-consumption/moderate-binge-drinking

Negin, J., Martiniuk, A., Cumming, R. G., Naidoo, N., Phaswana-Mafuya, N., Madurai, L., ... \& Kowal, P. (2012). Prevalence of HIV and chronic comorbidities among older adults. AIDS (London, England), 26(0 1), S55. 
Nielsen. (2015) The new American vanguard: Latinos 50+ are healthy, wealthy, and wise. Retrieved from http://www.nielsen.com/us/en/insights/reports/ 2015/the-new-american-vanguard-latinos-50-are-healthy-wealthy-andwise.html

Ng, A. C. Y., Phillips, D. R., \& Lee, W. K. M. (2002). Persistence and challenges to filial piety and informal support of older persons in a modern Chinese society: A case study in Tuen Mun, Hong Kong. Journal of Aging Studies, 16(2), 135-153.

Nguyen, N., \& Holodniy, M. (2008). HIV infection in the elderly. Clinical Interventions in Aging, 3(3), 453.

Nonnemaker, J. M., McNeely, C. A., \& Blum, R. W. (2003). Public and private domains of religiosity and adolescent health risk behaviors: Evidence from the National Longitudinal Study of Adolescent Health. Social Science and Medicine, 57(11), 2049-2054. doi:10.1016=S0277-9536(03)00096-0

Norris, A. E., \& Ford, K. (1994). Associations between condom experiences and beliefs, intentions, and use in a sample of urban, low-income, AfricanAmerican and Hispanic youth. AIDS Education and Prevention, 6(1), 2739.

Ochoa, S. C., \& Sampalis, J. (2014). Risk perception and vulnerability to STIs and HIVIAIDS among immigrant Latin-American women in Canada. Culture, Health \& Sexuality, 16(4), 412-425.

Ohio University (2007, April 26). One-third of sexually active older adults with HIVIAIDS has unprotected sex. Science Daily. Retrieved from http://www.sciencedaily.com-/releases/2007/04/070425122157.htm

Overbeek, G., Stattin, H., Vermulst, A., Ha, T., \& Engels, R. C. (2007). Parentchild relationships, partner relationships, and emotional adjustment: A birth-to-maturity prospective study. Developmental Psychology, 43(2), 429.

Paltiel, A. D., Weinstein, M. C., Kimmel, A. D., Seage, G. R., Losina, E., Zhang, H... Walensky, R. P. (2005). Expanded screening for HIV in the United States-an analysis of cost-effectiveness. New England Journal of Medicine, 352(6), 586-595. doi:10.1056/NEJMsa042088

Pasick, R. J., Barker, J. C., Otero-Sabogal, R., Burke, N. J., Joseph, G., \& Guerra, C. (2009). Intention, subjective norms, and cancer screening in the context of relational culture. Health Education \& Behavior, 36(1), 91S110S. doi: 10.1177/1090198109338919 
Peragallo, N. (1996). Latino women and AIDS risk. Public Health Nursing, 13(3), 217-222.

Perrino, T., González-Soldevilla, A., Pantin, H., \& Szapocznik, J. (2000). The role of families in adolescent HIV prevention: A review. Clinical Child and Family Psychology Review, 3(2), 81-96.

Pew Research Center. (2017). How the U.S. Hispanic population is changing. Retrieved from http://www.pewresearch.org/fact-tank/2017/09/18/how-theu-s-hispanic- population-is-changing/

Pew Research Center. (2014). Fact Tank. With few new arrivals, Census lowers Hispanic population projections. Retrieved from http://www.pewresearch.org/fact-tank/2014/12/16/with-fewer-new-arrivalscensus-lowers-hispanic-population-projections-2/

Pilowsky, D. J., \& Wu, L. T. (2015). Sexual risk behaviors and HIV risk among Americans aged 50 years or older: a review. Substance Abuse and Rehabilitation, 6, 51.

Pinel, A. (1994). Besides carnival and soccer: Reflections about AIDS in Brazil. In P. Wijeyaratne, J. Roberts, J. Kitts and L. Arsenault (eds) Gender, health, and sustainable development: A Latin American perspective (Ottawa, Canada: International Development Research Center), pp. 6171.

Pinkerton, S. D., Bogart, L. M., Howerton, D., Snyder, S., Becker, K., \& Asch, S. M. (2010). Cost of rapid HIV testing at 45 US hospitals. AIDS Patient Care and STDs, 24(7), 409-413.

Plant, E. A., \& Sachs-Ericsson, N. (2004). Racial and ethnic differences in depression: the roles of social support and meeting basic needs. Journal of Consulting and Clinical Psychology, 72(1), 41.

Poynten, I. M., Grulich, A. E., \& Templeton, D. J. (2013). Sexually transmitted infections in older populations. Current Opinion in Infectious Diseases, 26(1), 80-85.

Pleck, J. H., Sonenstein, F. L., \& Ku, L. C. (1993). Masculinity ideology: Its impact on adolescent males' heterosexual relationships. Journal of Social Issues, 49(3), 11-29.

Pratt, G., Gascoyne, K., Cunningham, K., \& Tunbridge, A. (2010). Human immunodeficiency virus (HIV) in older people. Age and Ageing, 39(3), 289294. 
Pulerwitz, J., Amaro, H., Jong, W. D., Gortmaker, S. L., \& Rudd, R. (2002). Relationship power, condom use and HIV risk among women in the USA. AIDS Care, 14(6), 789-800.

Raffaelli, M., \& Green, S. (2003). Parent-adolescent communication about sex: Retrospective reports by Latino college students. Journal of Marriage and Family, 65(2), 474-481.

Raffaelli, M., \& Suarez-Al-Adam, M. (1998). Reconsidering the HIVIAIDS prevention needs of Latino women in the United States. Retrieved from http://digitalcommons.unl.edu/cgi/viewcontent.cgi?article=1083\&context=p sychfacpub

Rajabiun, S., Rumptz, M. H., Felizzola, J., Frye, A., Relf, M., Yu, G., \& Cunningham, W. E. (2008). The impact of acculturation on Latinos' perceived barriers to HIV primary care. Ethnicity \& Disease, 18(4), 403.

Raphael, D. (2004). Introduction to the social determinants of health. Social determinants of health: Canadian Perspectives, 1-18.

Ramirez, J. R., Crano, W. D., Quist, R., Burgoon, M., Alvaro, E. M., \& Grandpre, J. (2004). Acculturation, familism, parental monitoring, and knowledge as predictors of marijuana and inhalant use in adolescents. Psychology of Addictive Behaviors, 18(1), 3.

Ridenour, T. A., Greenberg, M. T., \& Cook, E. T. (2006). Structure and validity of people in my life: A self-report measure of attachment in late childhood. Journal of Youth and Adolescence, 35(6), 1037-1053.

Riley, E. D., Gandhi, M., Hare, C. B., Cohen, J., \& Hwang, S. W. (2007). Poverty, unstable housing, and HIV infection among women living in the United States. Current HIV/AIDS Reports, 4(4), 181-186.

Rios-Ellis, B., Espinoza, L., Bird, M., Garcia, M., D'Anna, L. H., Bellamy, L., \& Scolari, R. (2010). Increasing HIV-related knowledge, communication, and testing intentions among Latinos: Protege tu Familia: Hazte la Prueba. Journal of Health Care for the Poor and Underserved, 21(3), 148-168.

Robles, R. R., Matos, T. D., Deren, S., Colón, H. M., Sahai, H., Marrero, C. A., ... \& Shepard, E. W. (2006). Drug treatment disparities among Hispanic drugusing women in Puerto Rico and New York City. Health Policy, 75(2), 159169. 
Rogler, L. H., Cortes, D. E., \& Malgady, R. G. (1991). Acculturation and mental health status among Hispanics: Convergence and new directions for research. American Psychologist, 46(6), 585-597.

Rojas, P., Huang, H., Li, T., Ravelo, G. J., Sanchez, M., Dawson, C., ... \& De La Rosa, M. (2016). Sociocultural determinants of risky sexual behaviors among adult Latinas: a longitudinal study of a community-based sample. International Journal of Environmental Research and Public Health, 13(11), 1164.

Rojas-Guyler, L., Ellis, N., \& Sanders, S. (2005). Acculturation, health protective sexual communication, and HIVIAIDS risk behavior among Hispanic women in a large Midwestern city. Health Education \& Behavior, 32(6), 767-779.

Romero, G., Arguelles, L., \& Rivero, A. M. (1993). Latinas and HIV infection/AIDS: Reflections on impacts, dilemmas and struggles. Wings of gauze: Women of Color and the Experience of Health and IIIness, 340352.

Romo, L. F., Bravo, M., \& Tschann, J. M. (2014). The effectiveness of a joint mother-daughter sexual health program for Latina early adolescents. Journal of Applied Developmental Psychology, 35(1), 1-9.

Romo, L. F., Lefkowitz, E. S., Sigman, M., \& Au, T. K. (2002). A longitudinal study of maternal messages about dating and sexuality and their influence on Latino adolescents. Journal of Adolescent Health, 31(1), 59-69.

Gloria, A. M., Ruiz, E. L., \& Castillo, E. M. (2004). Counseling and psychotherapy with Latino and Latina clients. Practicing multiculturalism: Affirming Diversity in Counseling and Psychology, 167-189.

Sabogal, F., Marín, G., Otero-Sabogal, R., Marín, B. V., \& Perez-Stable, E. J. (1987). Hispanic familism and acculturation: What changes and what doesn't?. Hispanic Journal of Behavioral Sciences, 9(4), 397-412.

Sánchez, M., Rice, E., Stein, J., Milburn, N. G., \& Rotheram-Borus, M. J. (2010). Acculturation, coping styles, and health risk behaviors among HIV positive Latinas. AIDS and Behavior, 14(2), 401-409.

Sanders, G. D., Bayoumi, A. M., Holodniy, M., \& Owens, D. K. (2008). Costeffectiveness of HIV screening in patients older than 55 years of age. Annals of Internal Medicine, 148(12), 889-903. 
Santa Maria, D., Markham, C., Bluethmann, S., \& Mullen, P. D. (2015). Parentbased adolescent sexual health interventions and effect on communication outcomes: a systematic review and meta-analyses. Perspectives on Sexual and Reproductive Health, 47(1), 37-50.

Savasta, A. M. (2004). HIV: Associated transmission risks in older adults-an integrative review of the literature. Journal of Association of Nurses AIDS Care, 15(1), 50-59.

Schensul, J. J., Singh, S. K., Gupta, K., Bryant, K., \& Verma, R. (2010). Alcohol and HIV in India: a review of current research and intervention. AIDS and Behavior, 14, 1-7.

Schick, V., Herbenick, D., Reece, M., Sanders, S. A., Dodge, B., Middlestadt, S. E., \& Fortenberry, J. D. (2010). Sexual behaviors, condom use, and sexual health of Americans over 50: implications for sexual health promotion for older adults. The Journal of Sexual Medicine, 7(s5), 315329.

Schmidt, S., Nachtigall, C., Wuethrich-Martone, O., \& Strauss, B. (2002). Attachment and coping with chronic disease. Journal of Psychosomatic Research, 53(3), 763-773.

Shedlin, M. G., Decena, C. U., \& Oliver-Velez, D. (2005). Initial acculturation and HIV risk among new Hispanic immigrants. Journal of the National Medical Association, 97(7 Suppl), 32S.

Sheehan, D. M., Trepka, M. J., Fennie, K. P., \& Maddox, L. M. (2015). Rate of new HIV diagnoses among Latinos living in Florida: Disparities by country/region of birth. AIDS Care, 27(4), 507-511.

Sheehan, D. M., Trepka, M. J., Fennie, K. P., Prado, G., Madhivanan, P., Dillon, F. R., \& Maddox, L. M. (2017). Individual and neighborhood determinants of late HIV diagnosis among Latinos, Florida, 2007-2011. Journal of Immigrant and Minority Health, 19(4), 825-834.

Shuper, P. A., Joharchi, N., Irving, H., \& Rehm, J. (2009). Alcohol as a correlate of unprotected sexual behavior among people living with HIVIAIDS: review and meta-analysis. AIDS and Behavior, 13(6), 1021-1036.

Shuper, P. A., Neuman, M., Kanteres, F., Baliunas, D., Joharchi, N., \& Rehm, J. (2010). Causal considerations on alcohol and HIVIAIDS—a systematic review. Alcohol and alcoholism, 45(2), 159-166. 
Simone, M. J., \& Appelbaum, J. (2008). HIV in older adults. Geriatrics, 63(12), 612.

Sinicrope, P. S., Patten, C. A., Clark, L. P., Brockman, T. A., Frost, M. H., Petersen, L. R., ... \& Sellers, T. A. (2009). Adult daughters' reports of breast cancer risk reduction and early detection advice received from their mothers: an exploratory study. Psycho-Oncology, 18(2), 169-178.

Skiest, D. J., \& Keiser, P. (1997). Human immunodeficiency virus infection in patients older than 50 years: a survey of primary care physicians' beliefs, practices, and knowledge. Archives of Family Medicine, 6(3), 289.

Smith, R. D., Delpech, V. C., Brown, A. E., \& Rice, B. D. (2010). HIV transmission and high rates of late diagnoses among adults aged 50 years and over. Aids, 24(13), 2109-2115.

Statistical Atlas. (2015). Languages in Miami-Dade County Florida. Retrieved from https://statisticalatlas.com/county/Florida/Miami-DadeCounty/Languages

Steensland, B., Robinson, L. D., Wilcox, W. B., Park, J. Z., Regnerus, M. D., \& Woodberry, R. D. (2000). The measure of American religion: Toward improving the state of the art. Social Forces, 79(1), 291-318.

Stewart, S. L., Rakowski, W., \& Pasick, R. J. (2009). Behavioral constructs and mammography in five ethnic groups. Health Education \& Behavior, 36(5_suppl), 36S-54S.

Timmins, C. L. (2002). The impact of language barriers on the health care of Latinos in the United States: A review of the literature and guidelines for practice. Journal of Midwifery \& Women's Health, 47(2), 80-96. doi: 10.1016/S1526-9523(02)00218-0

Tolma, E., Batterton, C., Hamm, R. M., Thompson, D., \& Engelman, K. K. (2012). American Indian women and screening mammography: Findings from a qualitative study in Oklahoma. American Journal of Health Education, 43(1), 18-30.

Ugarte-Gil C., Ponce M., Zamudio C., Canaza L., Samalvides F., Seas C. (2013). Knowledge about HIV prevention and transmission among recently diagnosed tuberculosis patients: A cross sectional study. BMC Public Health, 123, 1237. doi:10.1186/1471-2458-13-1237 
Unger, J. B., Gallaher, P., Shakib, S., Ritt-Olson, A., Palmer, P. H., \& Johnson, C. A. (2002). The AHIMSA acculturation scale: a new measure of acculturation for adolescents in a multicultural society. The Journal of Early Adolescence, 22(3), 225-251.

Unger, J. B., Ritt-Olson, A., Teran, L., Huang, T., Hoffman, B. R., \& Palmer, P. (2002). Cultural values and substance use in a multiethnic sample of California adolescents. Addiction Research and Theory, 10(3), 257-279

Unger, J. B., Shakib, S., Gallaher, P., Ritt-Olson, A., Mouttapa, M., \& Palmer, P. H. (2006). Cultural/interpersonal values and smoking in an ethnically diverse sample of Southern California adolescents. Journal of Cultural Diversity, 13(1), 55-63.

United States Census Bureau (US Census Bureau). (2015). New Census Bureau Report Analyzes U.S. Population Projections. Retrieved from http://www.census.gov/newsroom/press-releases/2015/cb15-tps16.html

United States Census Bureau (US Census Bureau). (2016). Educational Attainment in the United States: 2010. Retrieved from https://www.census.gov/data/tables/2010/demo/educational-attainment/ cps-detailed-tables.html

Upchurch, D. M., Aneshensel, C. S., Mudgal, J., \& McNeely, C. S. (2001). Sociocultural contexts of time to first sex among Hispanic adolescents. Journal of Marriage and Family, 63(4), 1158-1169.

U.S. Department of Health and Human Services (HHS), AIDS.gov. A Timeline of AIDS. (2011). Retrieved from http://aids.gov/hiv-aids-basics/hiv-aids101/aids-timeline/

Van Handel, M., Lyons, B., Oraka, E., Nasrullah, M., DiNenno, E., \& Dietz, P. (2015). Factors associated with time since last HIV test among persons at high risk for HIV infection, National Survey of Family Growth, 2006-2010. AIDS Patient Care and STDs, 29(10), 533-540.

Villarruel, A. M. (1998). Cultural influences on the sexual attitudes, beliefs, and norms of young Latina adolescents. Journal for Specialists in Pediatric Nursing, 3(2), 69-79.

Villarreal, R., Blozis, S. A., \& Widaman, K. F. (2005). Factorial invariance of a pan-hispanic familism scale. Hispanic Journal of Behavioral Sciences, 27(4), 409-425. 
Waller, E., Scheidt, C. E., \& Hartmann, A. (2004). Attachment representation and illness behavior in somatoform disorders. The Journal of Nervous and Mental Disease, 192(3), 200-209.

Warren, J. T., Harvey, S. M., \& Bovbjerg, M. L. (2011). Characteristics related to effective contraceptive use among a sample of nonurban Latinos. Perspectives on Sexual and Reproductive Health, 43(4), 255-262.

Washington, P. K., Burke, N. J., Joseph, G., Guerra, C., Pasick, R. J. (2009). Adult daughters' influence on mothers' health-related decision making: An expansion of the subjective norms construct. Health Education \& Behavior, 36(5), 129S-144S. doi:10.1177/1090198109338904

Weeks, M. R., Schensul, J. J., Williams, S. S., Singer, M., \& Grier, M. (1995). AIDS prevention for African-American and Latina women: Building culturally and gender-appropriate intervention. AIDS Education and Prevention, 7(3), 251-264.

Weinhardt, L. S., Carey, M. P., Johnson, B. T., \& Bickham, N. L. (1999). Effects of HIV counseling and testing on sexual risk behavior: A meta-analytic review of published research, 1985-1997. American Journal of Public Health, 89(9), 1397-1405.

Widman, L., Choukas-Bradley, S., Noar, S. M., Nesi, J., \& Garrett, K. (2016). Parent-adolescent sexual communication and adolescent safer sex behavior: a meta-analysis. Journal of the American Medical AssociationPediatrics, 170(1), 52-61.

Wight, D., \& Fullerton, D. (2013). A review of interventions with parents to promote the sexual health of their children. Journal of Adolescent Health, 52(1), 4-27.

World Health Organization. (2010a). A conceptual framework for action on the social determinants of health. Retrieved from http://www.who.int/ sdhconference/resources/ConceptualframeworkforactiononSDH_eng.pdf

World Health Organization. (2010b). Definition of an older or elderly person. Retrieved from http://www.who.int/healthinfo/survey/ageingdefnolder/en/ index.html

Zambrana, R. E., Cornelius, L. J., Boykin, S. S., \& Lopez, D. S. (2004). Latinas and HIVIAIDS Risk Factors: Implications for Harm Reduction Strategies. American Journal of Public Health, 94(7), 1152-1158. 
Zuckerman, S., Waidmann, T. A., \& Lawton, E. (2011). Undocumented immigrants, left out of health reform, likely to continue to grow as share of the uninsured. Health Affairs, 30(10), 1997-2004. 
APPENDICES 
Appendix A

PERSPECTIVE ON MOTHER/DAUGHTER ATTACHMENT

PERSPECTIVE ON MOTHER/DAUGHTER ATTACHMENT: Some of the following statements ask about your feelings about your [IFMD]. answer the questions for the one who is participating with you.

B1. My [IFMD] respects my feelings?

1 Almost never or never true

2 Not very often true

3 Sometimes true

4 Often True

5 Almost always/always true

B2. I feel my [IFMD] does a good job as my [IFMD].

1 Almost never or never true

2 Not very often true

3 Sometimes true

4 Often True

5 Almost always/always true

B3. I wish I had a different [IFMD].

1 Almost never or never true

2 Not very often true

3 Sometimes true

4 Often True

5 Almost always/always true

B4. My [IFMD] accepts me as I am.

1 Almost never or never true

2 Not very often true

3 Sometimes true

4 Often True

5 Almost always/always true

B5. I like to get my [IFMD] point of view on things I'm concerned about.

1 Almost never or never true

2 Not very often true

3 Sometimes true

4 Often True

5 Almost always/always true 
B6. I feel it's no use letting my feelings show around my [IFMD].

1 Almost never or never true

2 Not very often true

3 Sometimes true

4 Often True

5 Almost always/always true

B7. My [IFMD] can tell when I'm upset about something.

1 Almost never or never true

2 Not very often true

3 Sometimes true

4 Often True

5 Almost always/always true

B8. Talking over my problems with my [IFMD] makes me feel ashamed or foolish.

1 Almost never or never true

2 Not very often true

3 Sometimes true

4 Often True

5 Almost always/always true

B9. My [IFMD] expects too much from me.

1 Almost never or never true

2 Not very often true

3 Sometimes true

4 Often True

5 Almost always/always true

B10. I get upset easily around my [IFMD].

1 Almost never or never true

2 Not very often true

3 Sometimes true

4 Often True

5 Almost always/always true

B11. I get upset a lot more than my [IFMD] knows about.

1 Almost never or never true

2 Not very often true

3 Sometimes true

4 Often True

5 Almost always/always true 
B12. When we discuss things, my [IFMD] cares about my point of view.

1 Almost never or never true

2 Not very often true

3 Sometimes true

4 Often True

5 Almost always/always true

B13. My [IFMD] trusts my judgment.

1 Almost never or never true

2 Not very often true

3 Sometimes true

4 Often True

5 Almost always/always true

B14. My [IFMD] has her own problems, so I don't bother her with mine.

1 Almost never or never true

2 Not very often true

3 Sometimes true

4 Often True

5 Almost always/always true

B15. My [IFMD] helps me to understand myself better.

1 Almost never or never true

2 Not very often true

3 Sometimes true

4 Often True

5 Almost always/always true

B16. I tell my [IFMD] about my problems and troubles.

1 Almost never or never true

2 Not very often true

3 Sometimes true

4 Often True

5 Almost always/always true

B17. I feel angry with my [IFMD].

1 Almost never or never true

2 Not very often true

3 Sometimes true

4 Often True

5 Almost always/always true 
B18. I don't get much attention from my [IFMD].

1 Almost never or never true

2 Not very often true

3 Sometimes true

4 Often True

5 Almost always/always true

B19. My [IFMD] helps me to talk about my difficulties.

1 Almost never or never true

2 Not very often true

3 Sometimes true

4 Often True

5 Almost always/always true

B20. My [IFMD] understands me.

1 Almost never or never true

2 Not very often true

3 Sometimes true

4 Often True

5 Almost always/always true

B21. When I am angry about something, my [IFMD] tries to be understanding.

1 Almost never or never true

2 Not very often true

3 Sometimes true

4 Often True

5 Almost always/always true

B22. I trust my [IFMD].

1 Almost never or never true

2 Not very often true

3 Sometimes true

4 Often True

5 Almost always/always true

B23. My [IFMD] doesn't understand what I'm going through these days.

1 Almost never or never true

2 Not very often true

3 Sometimes true

4 Often True

5 Almost always/always true 
B24. I can count on my [IFMD] when I need to get something off my chest.

1 Almost never or never true

2 Not very often true

3 Sometimes true

4 Often True

5 Almost always/always true

B25. If my [IFMD] knows something is bothering me, she asks me about it.

1 Almost never or never true

2 Not very often true

3 Sometimes true

4 Often True

5 Almost always/always true 
Appendix B

\section{ACCULTURATION}

ACCULTURATION: We are almost at the end of the questionnaire. The next questions ask about the differences and similarities that you notice between your country of origin and the United States. Included are questions on: where each family member has lived for most of their life, language preferences, and values.

Q1. How do you describe yourself? Check all that apply. (Check all that apply)

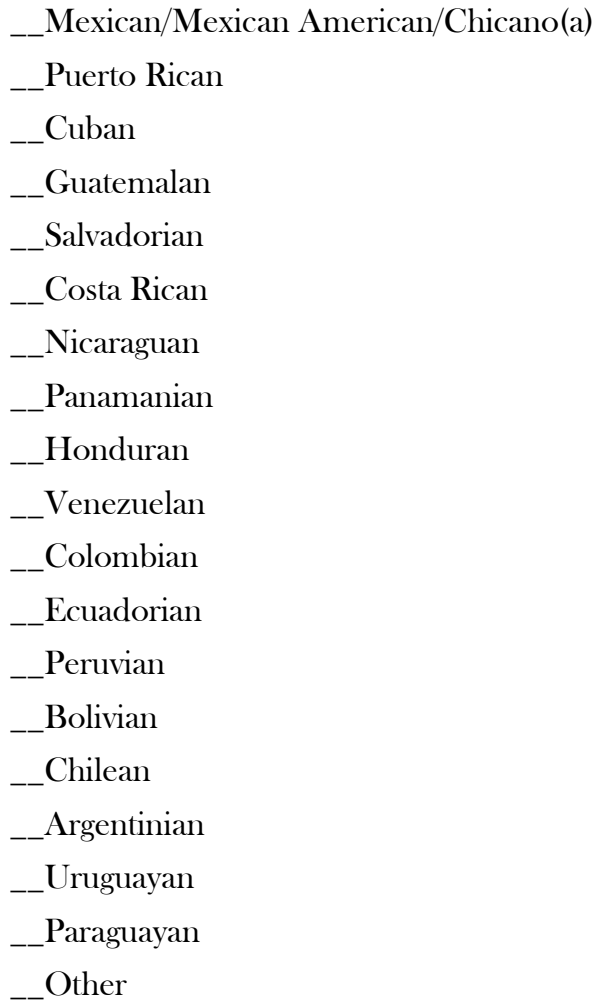

Q1A. If "Other", specify:

Q2. How well do you speak Spanish? (Choose one)

1 Not at all

2 Fair

3 Good

4 Excellent 
Q3. How well do you read Spanish? (Choose one)

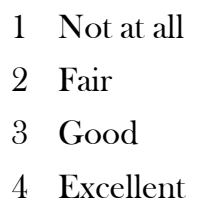

Q4. How well do you write Spanish? (Choose one)

$1 \quad$ Not at all

2 Fair

3 Good

4 Excellent

Q5. How well do you speak English? (Choose one)

$\begin{array}{ll}1 & \text { Not at all } \\ 2 & \text { Fair } \\ 3 & \text { Good } \\ 4 & \text { Excellent }\end{array}$

Q6. How well do you read English? (Choose one)

$\begin{array}{ll}1 & \text { Not at all } \\ 2 & \text { Fair } \\ 3 & \text { Good } \\ 4 & \text { Excellent }\end{array}$

Q7. How well do you write English? (Choose one)

$\begin{array}{ll}1 & \text { Not at all } \\ 2 & \text { Fair } \\ 3 & \text { Good } \\ 4 & \text { Excellent }\end{array}$

Q8. How familiar would you say you are with fine art and fine artists of your Latin American country? (PROBE: Painters, actors.) (Choose one)

1 I don't know anything about these

2 I know very little about these

3 I know something about these

4 I know a lot about these

Q9. How familiar would you say you are with fine art and fine artists of the United States? (Choose one)

1 I don't know anything about these

2 I know very little about these

3 I know something about these

4 I know a lot about these 
Q10. How familiar would you say you are with history and politics of your Latin American country? (Choose one)

1 I don't know anything about these

2 I know very little about these

3 I know something about these

4. I know a lot about these

Q11. How familiar would you say you are with history and politics of the United States? (Choose one)

1 I don't know anything about these

2 I know very little about these

3 I know something about these

4 I know a lot about these

Q12. How familiar would you say you are with cultural legends and symbols of your Latin American country? (PROBE: like the flag.) (Choose one)

1 I don't know anything about these

2 I know very little about these

3 I know something about these

4 I know a lot about these

Q13. How familiar would you say you are with cultural legends and symbols of the United States? (Choose one)

1 I don't know anything about these

2 I know very little about these

3 I know something about these

4 I know a lot about these

Q14. How familiar would you say you are with popular entertainers of your Latin American country? (Choose one)

1 I don't know anything about these

2 I know very little about these

3 I know something about these

4 I know a lot about these

Q15. How familiar would you say you are with popular entertainers of the United States? (Choose one)

1 I don't know anything about these

2 I know very little about these

3 I know something about these

4 I know a lot about these 
Q16. In choosing a boyfriend/girlfriend, I: (Choose one)

$\begin{array}{ll}1 & \text { Accept only Latino(a) } \\ 2 & \text { Prefer a Latino(a) } \\ 3 & \text { Have no preference } \\ 4 & \text { Prefer White } \\ 5 & \text { Accept only a White } \\ 6 & \text { Prefer someone that is neither Latino(a) or White }\end{array}$

Q17. If I could choose a boyfriend/girlfriend for a member of my family, I would: (Choose one)

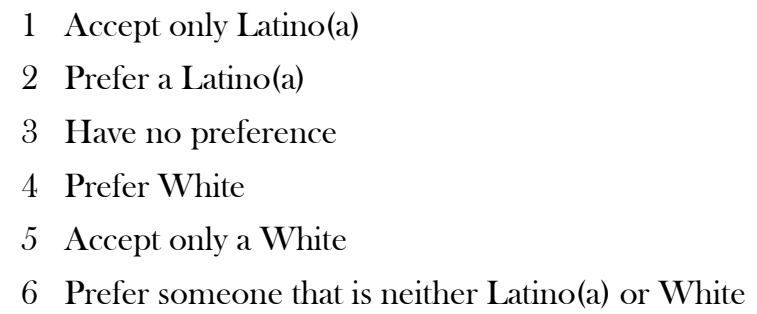

Q18. In choosing a partner for marriage, I will: (Choose one)

1 Accept only Latino(a)

2 Prefer a Latino(a)

3 Have no preference

4 Prefer White

5 Accept only a White

6 Prefer someone that is neither Latino(a) or White

\section{Language}

Q19. What language do you use when you speak to your parents? (Choose one)

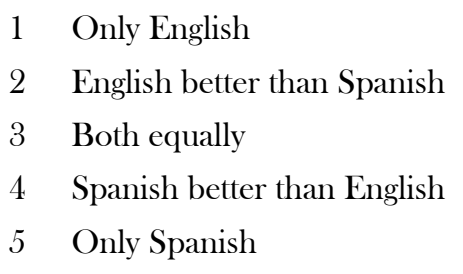

Q20. In what language are the TV programs you usually watch with your friends (Choose one)

$\begin{array}{ll}1 & \text { Only English } \\ 2 & \text { English better than Spanish } \\ 3 & \text { Both equally } \\ 4 & \text { Spanish better than English } \\ 5 & \text { Only Spanish }\end{array}$


Q21. I prefer to watch TV in... (Choose one)

$\begin{array}{ll}1 & \text { Only English } \\ 2 & \text { English better than Spanish } \\ 3 & \text { Both equally } \\ 4 & \text { Spanish better than English } \\ 5 & \text { Only Spanish }\end{array}$

Q22. In what language are the radio programs and music you usually listen to when you are with your friends? (Choose one)

$\begin{array}{ll}1 & \text { Only English } \\ 2 & \text { English better than Spanish } \\ 3 & \text { Both equally } \\ 4 & \text { Spanish better than English } \\ 5 & \text { Only Spanish }\end{array}$

Q23. In what language are the radio programs and music you usually listen to when you are by yourself? (Choose one)

$\begin{array}{ll}1 & \text { Only English } \\ 2 & \text { English better than Spanish } \\ 3 & \text { Both equally } \\ 4 & \text { Spanish better than English } \\ 5 & \text { Only Spanish }\end{array}$




\section{Appendix C}

\section{DRINKING}

\section{DRINKING: This section asks you specific questions about your recent drinking. For each item tell me the answer that best describes you.}

G1. How often did you drink beer during the last 3 months? (Choose one)

$$
\begin{array}{ll}
1 & \text { Never } \\
2 & \text { Several times } \\
3 & \text { About once a month } \\
4 & \text { Several times a month } \\
5 & 1 \text { to } 2 \text { days a week } \\
6 & 3 \text { to } 4 \text { days a week } \\
7 & 5 \text { to } 6 \text { days a week } \\
8 & \text { Everyday } \\
9 & \text { Other }
\end{array}
$$

G1A. If "Other", specify:

G2. What was the usual amount of beer you had on any typical day when you drank beer during the past 3 months? (Choose one)

1 1-2 bottles or cans (12 oz.)

2 3-5 bottles or cans (12 oz.)

3 About 1 six pack

4 About 2 six packs

53 six packs or more

G3. How often did you drink beer during the last 12 months? (Choose one)

$$
\begin{array}{ll}
1 & \text { Never } \\
2 & \text { Several times } \\
3 & \text { About once a month } \\
4 & \text { Several times a month } \\
5 & 1 \text { to } 2 \text { days a week } \\
6 & 3 \text { to } 4 \text { days a week } \\
7 & 5 \text { to } 6 \text { days a week } \\
8 & \text { Everyday } \\
9 & \text { Other }
\end{array}
$$

G3A. If "Other", specify: 
G4. What was the usual amount of beer you had on any typical day when you drank beer during the past 12 months? (Choose one)

1 1-2 bottles or cans (12 oz.)

2 3-5 bottles or cans (12 oz.)

3 About 1 six pack

4 About 2 six packs

53 six packs or more

G5. Do you drink light beer or regular beer? (Choose one)

1 Light Beer

2 Regular Beer

3 Neither light nor regular

4 Both light and regular

G6. About how often did you drink hard liquor (vodka, rum, whiskey) during the past 3 months? (Choose one)

$\begin{array}{ll}1 & \text { Never } \\ 2 & \text { Several times } \\ 3 & \text { About once a month } \\ 4 & \text { Several times a month } \\ 5 & 1 \text { to } 2 \text { days a week } \\ 6 & 3 \text { to } 4 \text { days a week } \\ 7 & 5 \text { to } 6 \text { days a week } \\ 8 & \text { Everyday } \\ 9 & \text { Other }\end{array}$

G6A. If "Other", specify:

G7. What was the usual amount of hard liquor (vodka, rum, whiskey) you had on any typical day when you drank liquor during the last 3 months? (Choose one)

$\begin{array}{ll}1 & \text { 1-2 drinks } \\ 2 & 3-4 \text { drinks } \\ 3 & 5 \text {-7 drinks } \\ 4 & 8 \text {-10 drinks } \\ 5 & \text { About } 12 \text { drinks } \\ 6 & \text { About } 1 \text { pint } \\ 7 & \text { About } 2 \text { pints } \\ 8 & 3 \text { pints or more }\end{array}$


G8. About how often did you drink hard liquor (vodka, rum, whiskey) during the past 12 months? (Choose one)

$\begin{array}{ll}1 & \text { Never } \\ 2 & \text { Several times } \\ 3 & \text { About once a month } \\ 4 & \text { Several times a month } \\ 5 & 1 \text { to } 2 \text { days a week } \\ 6 & 3 \text { to } 4 \text { days a week } \\ 7 & 5 \text { to } 6 \text { days a week } \\ 8 & \text { Everyday } \\ 9 & \text { Other }\end{array}$

G8A. If "Other", specify:

G9. What was the usual amount of hard liquor (vodka, rum, whiskey) you had on any typical day when you drank liquor during the last 12 months? (Choose one)

$\begin{array}{ll}1 & 1-2 \text { drinks } \\ 2 & 3-4 \text { drinks } \\ 3 & 5-7 \text { drinks } \\ 4 & 8-10 \text { drinks } \\ 5 & \text { About } 12 \text { drinks } \\ 6 & \text { About } 1 \text { pint } \\ 7 & \text { About } 2 \text { pints } \\ 8 & 3 \text { pints or more }\end{array}$

G10. When you drink hard liquor, how many ounces do you mix in each drink (Choose one)

$\begin{array}{ll}1 & 1 \mathrm{oz} . \\ 2 & 2 \mathrm{oz} .(1 \mathrm{shot}) \\ 3 & 3 \mathrm{oz} . \\ 4 & 4 \mathrm{oz} .(1 / 2 \mathrm{cup}) \\ 5 & \text { More than } 4 \mathrm{oz} .\end{array}$


G11. About how often did you drink wine during the past 3 months? (Choose one)

$\begin{array}{ll}1 & \text { Never } \\ 2 & \text { Several times } \\ 3 & \text { About once a month } \\ 4 & \text { Several times a month } \\ 5 & 1 \text { to } 2 \text { days a week } \\ 6 & 3 \text { to } 4 \text { days a week } \\ 7 & 5 \text { to } 6 \text { days a week } \\ 8 & \text { Everyday } \\ 9 & \text { Other }\end{array}$

G11A. If "Other", specify:

G12. What was the usual amount of wine you had on a typical day when you drank wine during the past 3 months? (Choose one)

\author{
$1 \quad 1-3$ glasses \\ 2 4-5 glasses \\ 3 About 6 glasses (one bottle) \\ 4 About 9 glasses \\ 5 About 2 bottles (12 glasses)
}

G13. About how often did you drink wine during the past 12 months? (Choose one)

$\begin{array}{ll}1 & \text { Never } \\ 2 & \text { Several times } \\ 3 & \text { About once a month } \\ 4 & \text { Several times a month } \\ 5 & 1 \text { to } 2 \text { days a week } \\ 6 & 3 \text { to } 4 \text { days a week } \\ 7 & 5 \text { to } 6 \text { days a week } \\ 8 & \text { Everyday } \\ 9 & \text { Other }\end{array}$

G13A. If "Other", specify: 
G14. What was the usual amount of wine you had on a typical day when you drank wine during the past 12 months? (Choose one)
$1 \quad 1-3$ glasses
$2 \quad 4-5$ glasses
3 About 6 glasses (one bottle)
4 About 9 glasses
5 About 2 bottles (12 glasses)
6 Three bottles or more

G15. How often did you drink wine coolers (or something similar) during the last 3 months? (Choose one)

\author{
1 Never in the past 3 months \\ 2 Several times in the past 3 months \\ 3 About once a month \\ 4 Several times a month \\ 5 1-2 days a week \\ 6 3-4 days a week \\ 7 5-6 days a week \\ 8 Everyday \\ 9 Other
}

G15A. If "Other", specify:

G16. What was the usual amount of wine coolers (or something similar) you had on any typical day when you drank wine coolers during the past 3 months? (Choose one)

$1 \quad 1-2$ bottles (12 oz.)

2 3-5 bottles (12 oz.)

3 About 1 six pack

4 About 2 six packs

53 six packs or more 
VITA

\section{GIRA J. RAVELO}

1997

BA, Psychology/Spanish

University of New Mexico

Albuquerque, NM

2012:

MSW, Social Work

Florida International University

Miami, FL

2016:

Doctoral Candidate in Social Welfare

Florida International University

Miami, FL

Ravelo, G. J., Kanamori, M., Rojas, P., De La Rosa, M. (2014). Associations between Living Arrangements and Health Behaviors among Older Latina Women. Poster presented at the National Institute on Minority Health and Health Disparities Grantees' Conference, formerly known as International Symposium on Minority Health and Health Disparities (ISMHHD), National Harbor, MD.

Kanamori, M., Rojas, P, Ravelo, G. J., Jaramillo, M., De La Rosa, M. (2014). Latino dyadic mother/daughter attachment: Emotional abuse and drug use. Poster presented at the National Institute on Minority Health and Health Disparities Grantees' Conference, formerly known as International Symposium on Minority Health and Health Disparities (ISMHHD), National Harbor, MD.

De La Rosa, M., Huang, H., Rojas, P., Dillon, F. R., Lopez-Quintero, C., Li, T., \& Ravelo, G. J. (2015). Influence of mother-daughter attachment on substance use: A longitudinal study of a Latina community-based sample. Journal of Studies on Alcohol and Drugs, 76(2), 307-316. doi:10.15288/jsad.2015.76.307

Ravelo, G. J., Kanamori, M., Rojas, G. J., De La Rosa, M. (2015, June). Longitudinal analysis of changes in living arrangements and health related behaviors among older Latina women. Poster presented at the 2015 National Hispanic Science Network Conference, San Antonio, TX.

De La Rosa, M., Huang, H., Rojas, P., Dillon, F. R., Lopez-Quintero, C., Li, T., \& Ravelo, G. J (2015). Influence of Mother-Daughter Attachment on Substance Use: A Longitudinal Study of a Latina Community-Based Sample. Panel presentation at 
George Washington University, Milken Institute School of Public Health. Regional Latino Health Disparities Conference: Breaking Down Structural Barriers to Latino Health Equity, Washington DC.

Sanchez, M., Rojas, P., Li, T., Ravelo, G.J., Cyrus, E., Wang, W., Kanamori, M., Peragallo, N.P., \& De La Rosa, M. (2016). Evaluating a culturally tailored HIV risk reduction intervention among Latina immigrant farmworkers. World Medical \& Health Policy. 8(3), 245-262. doi: 10.1002/wmh3.193

Rojas, P., Li, T., Wang, W., Ravelo, G. J., Sanchez, M., De La Rosa, M. (2016, March). Correlates of Cervical Cancer Screening among Adult Latina Mothers and Daughters. Oral presentation at George Mason University, Center for Global Studies. Women's Health in Global Perspectives, Washington DC.

Ravelo, G. J., Sanchez, M., Rojas, P., Cyrus, E., De La Rosa, M. (2016, July). Gender Norms and HIV Protective Factors among Latinas in the Farmworker Community: Examining Mediating Effects of HIV Self Efficacy. Poster presentation at 2016 Latina Researchers Network \& Conference, San Antonio, TX.

Rojas, P., Li, T., Ravelo, G. J., Sanchez, M., Dawson, C., Sneij, A., Wang, W., Cyrus, E., Kanamori, M., \& De La Rosa, M. (2016). Correlates of cervical cancer screening among a non-clinical community based sample of adult Latino women: A five year follow up. Submitted to World Medical \& Health Policy.

Rojas, P., Huang, H., Li, T., Ravelo, G. J., Sanchez, M., Dawson, C., Brook, J., Kanamori, M., De La Rosa, M. (2016). Socio-Cultural Determinants of Risky Sexual Behaviors among Adult Latinas: A Longitudinal Study of a CommunityBased Sample. International Journal of Environmental Research and Public Health.

Sanchez, M., Ravelo, G.J., Cyrus, E., Dawson, C., Cano, M.A., Martinez, M., Romano, E., Rojas, P., Li., T., De La Rosa, M.R. Trajectories of Pre- to PostImmigration Religious Social Capital and its Impact on Drinking and Driving Risk Behaviors of Recent Latino Immigrants. Submitted to Journal of Latino/a Psychology.

Fava, N., Sanchez, M., Ravelo, G. J., Rojas, P., De La Rosa, M. (2017, January). Sexual Trauma and Wellbeing among Latinas: The Moderating Role of Gender Norms. Panel presentation at Society for Social Work Research $21^{\text {st }}$ Annual Conference: Ensure healthy Development for all Youth, New Orleans, LA. 